\title{
Polinômios Cúbicos Fibonacci, Decaimento de Geometria e Hiperbolicidade Induzida
}

\author{
Yong Su Han \\ Tese Apresentada \\ ao \\ Instituto de Matemática e Estatística \\ da \\ Universidade de São Paulo \\ para \\ Obtenção do Grau de Doutor \\ em \\ Matemática
}

Área de Concentração: Sistemas Dinâmicos

Orientador: Prof. Dr. Edson Vargas

\author{
Durante a elaboração deste trabalho \\ o autor recebeu apoio financeiro do CNPq \\ -São Paulo, Julho de 2004-
}




\section{Polinômios Cúbicos Fibonacci, Decaimento de Geometria e Hiperbolicidade Induzida}

Este exemplar corresponde à redação final da dissertaçào devidamente corrigida e defendida por Yong Su Han e aprovada pela comissão julgadora.

Säo Paulo, 6 de Julho de 2004.

Banca Examinadora:

-Prof. Dr. Edson Vargas (Orientador)

- IME-USP

-Prof. Dr. Albert Meads Fisher

- IME-USP

-Prof. Dr. Carlos Teobaldo Guiterrez Vidalon

- ICMC-USP

-Prof. Dr. Ronaldo Alves Garcia

- UFG

-Prof. Dr. Vanderlei Minori Horita

- UNESP 
aos meus filhos, presentes de Deus. 


\section{Resumo}

Neste trabalho consideramos os polinômios cúbicos eventualmente Fibonacci cujos pontos críticos são quadráticos e recorrentes e possuem o mesmo $\omega$-limite. Provamos que esses polinômios exibem decaimento exponencial de geometria. A seguir utilizamos este resultado para mostrar que todo polinômio cúbico que não apresenta retornos centrais e exibe decaimento exponencial de geometria induz uma aplicação de Markov hiperbólica. 


\section{Abstract}

On this work we study decay of geometry for cubic eventually Fibonacci maps with critical points of order 2 , recorrents and same $\omega$-limite. We also prove induced hyperbolicity for all bimodal maps with decay of geometry, but without central returns. This result is related to the absence of strange attractors. 


\section{Agradecimentos}

Sou muito grato a Deus que tem abençoado a minha vida acadêmica e espiritual para que eu pudesse estar neste momento da realização desta tese. Que só a Ele sejam dados a honra e o louvor através deste trabalho.

Agradeço de coração ao Prof. Edson Vargas pela sua sugestão do assunto deste trabalho e inestimável orientação nos momentos importantes, a ele sou profundamente grato.

Aos colegas Márcio e Liane, agradeço pelo companheirismo no trabalho e pelos momentos de amizade.

Aos colegas da igreja UBF, sou profundamente grato pela oração e palavras de carinho e coragem em todos os momentos. 


\section{Sumário}

1 Introdução 1

2 Conceitos Básicos e Resultados Principais 5

2.1 Partição de Yoccoz real . . . . . . . . . . . . . . . . . 5

2.2 Polinômios Cúbicos Fibonacci . . . . . . . . . . . . . . 7

2.3 Resultados Principais . . . . . . . . . . . . . . 10

3 Ferramentas Básicas $\quad 17$

3.1 Princípios de Koebe e Outros Fatos . . . . . . . . . . . . . . . . . 17

3.2 Ferramentas de análise complexa . . . . . . . . . . . . . 21

3.2 .1 Anéis e módulos de anéis . . . . . . . . . . . . 21

3.2 .2 Partição de Yoccoz complexa . . . . . . . . . . . . 24

4 Polinômios cúbicos Fibonacci e eventualmente Fibonacci $\quad 27$

4.1 Polinômios cúbicos Fibonacci f . . . . . . . . . . . . . . 27

4.2 Polinômios cúbicos eventualmente Fibonacci . . . . . . . . . . . . . 33

4.2.1 Tempos de retorno dos pontos críticos . . . . . . . . . 35

4.3 As sequiências de kneading dos polinômios cúbicos Fibonacci . . . . . . 39 
5.1 Crescimento dos anéis principais . . . . . . . . . . . . . . . 42

5.2 Anéis principais no caso eventualmente Fibonacci . . . . . . . . . . . 43

5.2.1 Polinômios eventualmente Fibonacci do tipo $F_{1} F_{2} \ldots \ldots$. . . . 44

5.2.2 Polinômios cúbicos eventualmente Fibonacci do tipo $F_{3} F_{4} F_{5} \quad \ldots \quad 52$

6 Hiperbolicidade Induzida $\quad 63$

6.1 Existência da Aplicação Induzida . . . . . . . . . . . . . . 63

6.2 Hiperbolicidade no Caso Básico . . . . . . . . . . . . 73

6.3 Hiperbolicidade no Caso Não-Básico . . . . . . . . . . . 76 


\section{Capítulo 1}

\section{Introdução}

Dado $I$ um intervalo compacto, uma aplicação contínua $f: I \rightarrow I$ é dita multimodal se I possui uma partição em um número finito de subintervalos restrita aos quais $f$ é estritamente monótona. Os pontos de fronteira destes subintervalos são extremos locais. Quando $f$ possui um único extremo local no interior de $I$ chamamo-la unimodal e quando possui dois extremos locais no interior de I chamamo-la bimodal.

A classe das aplicações multimodais inclui todos os polinômios reais, isto é: todos os polinômios de uma variável real com coeficientes reais como por exemplo $Q_{a}(x)=$ $-1+a\left(1-x^{2 l}\right), \quad P_{a b}(x)=a x^{3}+b x^{2}+(1-a-b) x$ ou $\quad Q_{a b}(x)=1-a x^{3}-b x^{2}-(1-a-b) x$.

Estamos interessados em aspectos métricos dos sistemas dinâmicos gerados pelas iterações de polinômios bimodais cúbicos $f=P_{a b}$ ou $f=Q_{a b}$, onde $a$ e $b$ são parâmetros reais. A presença de pontos críticos enriquece tais sistemas, pois estes possuem uma regiào muito contrativa próxima aos pontos críticos e regiões relativamente expansivas longe dos mesmos. Sendo assim, a estrutura das órbitas dos pontos críticos têm grande influência para a determinação de qual destes comportamentos predomina quando o 
sistema evolui. Como é usual em dinâmica utilizamos transformações de primeiro retorno como uma das ferramentas. Dado um tal polinômio $f$ consideramos o conjunto $P_{1}$ constituído de todos os pontos fixos de $f$. Definimos os intervalos $I_{0}$ e $J_{0}$ como sendo as componentes conexas de $[0,1] \backslash f^{-1}\left(P_{1}\right)$ que contêm, respectivamente, $c$ e $d$. Definimos também a transformação de primeiro retorno $\phi_{1}$ da união $I_{0} \cup J_{0}$. Assumindo que $c$ e $d$ são recorrentes existem intervalos $I_{1} \ni c$ e $J_{1} \ni d$ os quais são componentes conexas do domínio de $\phi_{1}$. Consideramos então a transformação de primeiro retorno $\phi_{2}$ de $I_{1} \cup J_{1}$. Novamente existem intervalos $I_{2} \ni c$ e $J_{2} \ni d$ os quais são componentes conexas do domínio de $\phi_{2}$. Procedendo indutivamente construímos duas seqüências de intervalos $I_{0} \supset I_{1} \supset I_{2} \supset \ldots \supset\{c\}$ e $J_{0} \supset J_{1} \supset J_{2} \supset \ldots \supset\{d\}$, onde $I_{n} \cup J_{n}$ é componentes do dominio de primeiro retorno a $I_{n-1} \cup J_{n-1} \mathrm{com}$ as suas respectivas transformações de primeiro retorno $\phi_{n}: I_{n} \cup J_{n} \rightarrow I_{n-1} \cup J_{n-1}$. Chamaremos de ramo à restrição de $\phi_{n}$ a cada componente conexa do seu domínio. E se o domínio de um ramo contém pontos criticos, chamamo-lo ramo crítico. Entào, os ramos de $\phi_{n}$ definidos em $I_{n}$ e $J_{n}$ são ramos críticos e unimodais cujas imagens estão contidas em $I_{n-1}$ ou $J_{n-1}$. Todos os outros ramos de $\phi_{n}$ nào possuem pontos críticos e aplicam seus domínios difeomorficamente sobre $I_{n-1}$ ou $J_{n-1}$.

Definimos as seqüências de razôes de escala em $c$ e em $d$ pondo

$$
\mu_{n}(c):=\frac{\left|I_{n}\right|}{\left|I_{n-1}\right|} \text { e } \quad \mu_{n}(d):=\frac{\left|J_{n}\right|}{\left|J_{n-1}\right|} .
$$

O comportamento destas sequências é importante para determinarmos a estrutura dos conjuntos $\omega$-limite $\omega(c)$ e $\omega(d)$. Quando ambas convergem para zero dizemos que $f$ exibe decaimento de geometria e quando isto não ocorre dizemos que $f$ possui geometria limitada. É natural esperar que decaimento de geometria esteja relacionado com algum 
tipo de hiperbolicidade e que isto dependa do número de iteradas que é necessário para que os pontos críticos retornem próximos deles mesmos. Mais precisamente temos que se $x=c$ ou $x=d$ então $\phi_{n+1}(x)=\phi_{n}^{k_{n}}(x)$ para algum $k_{n} \geq 1$. Se $k_{n}=1$ então $\phi_{n}(x) \in I_{n} \cup J_{n}$ e dizemos que este retorno é um retorno central. Os retornos centrais são os retomos mais rápidos possíveis e estão relacionados a uma forte recorrência do ponto $x$. Em uma sequência de retornos centrais consecutivos $\phi_{n_{1}}(x), \phi_{n_{2}}(x), \ldots, \phi_{n_{j}}(x)$ ocorre que $k_{n_{1}}=k_{n_{2}}=\ldots k_{n_{j}}=1$. Nestas sequências $\mu_{n_{k}}(x)\left(k=1,2, \ldots, n_{j}\right)$ nào decresce e até mesmo pode se aproximar de 1 . Estas sequências são chamadas de cascatas de retornos centrais.

No caso unimodal quadrático se excluirmos os retornos centrais, a saber, considerando todos os $n_{j}$ tais que $\phi_{n_{j}}(c)$, onde $c$ é o único ponto crítico, é não central ocorre que $\mu_{n_{j}}(c)$ tende a zero exponencialmente quando $j \rightarrow \infty$, veja [GS1] e [L]. A propriedade do ponto crítico ser quadrático desempenha um importante papel para este fato que pode não ocorrer para determinados tipos de recorrências se o ponto crítico é de ordem mais alta.

No caso bimodal cúbico introduzimos os polinômios de Fibonacci que dentre os que não possuem retornos centrais são aqueles cujos pontos críticos retornam mais rapidamente caracterizando a recorrência mais forte possível, e portanto a situaçào mais delicada, dentre os que não possuem retornos centrais. Neste trabalho descrevemos vários aspectos topológicos da dinâmica dos polinômios cúbicos Fibonacci. Provamos que os polinômios cúbicos que apresentam a combinatória de Fibonacci ou mais geralmente, a combinatória eventualmente Fibonacci, exibem decaimento exponencial de geometria. A partir desta propriedade obtemos uma aplicação de Markov induzida por $f$ que é hiperbólica confirmando assim que decaimento de geometria está relacionado à existência 
de alguma hiperbolicidade. A não-existência de atratores selvagens (veja a definição mais adiante) segue-se. Outras propriedades importantes também decorrem do decaimento de geometria.

No Capítulo 2 apresentamos alguns conceitos básicos como partição de Yoccoz real, transformações de primeiro retorno e polinômios cúbicos Fibonacci e eventualmente Fibonacci que são o tema principal deste trabalho. No mesmo capítulo enunciamos os resultados principais que provaremos. No Capitulo 3 apresentamos ferramentas importantes como os Princípios de Koebe que nos permitem fazer estimativas de distorção para a parte real da dinâmica e as noçôes de anéis e módulos de anéis úteis para a parte da dinâmica no plano complexo. No Capítulo 4 estudamos a existência de polinômios cúbicos Fibonacci e eventualmente Fibonacci, suas aplicações de primeiro retorno e a questão do menor tempo de retorno. No Capítulo 5 provamos o decaimento exponencial de geometria para os polinômios cúbicos eventualmente Fibonacci. No Capítulo 6 mostramos que polinômios cúbicos bimodais sem retornos centrais e com decaimento de geometria (dentre eles os eventualmente Fibonacci) induzem hiperbolicidade. 


\section{Capítulo 2}

\section{Conceitos Básicos e Resultados Principais}

\subsection{Partição de Yoccoz real}

Dado um polinômio bimodal cúbico $f=P_{a b}$ ou $f=Q_{a b}$, onde $a$ e $b$ são parâmetros reais consideraremos aspectos da sua dinâmica no intervalo [0,1] e também no plano complexo $\mathbb{C}$. As muitas ferramentas de análise de aplicações conformes serào cruciais para abordarmos questões importantes da dinâmica em $[0,1]$. Então introduziremos o conceito de partição de Yoccoz real a qual está relacionada com a partição de Yoccoz complexa que introduziremos mais tarde.

Seja $P_{-1}$ o conjunto constituído de todos os pontos fixos de $f$. Supondo que $P_{n}$ está determinado definimos $P_{n+1}:=f^{-1}\left(P_{n}\right)$. Deste modo obtemos uma sequência crescente $P_{-1} \subset P_{0} \subset P_{1} \subset \ldots$ Cada $P_{n}$ determina uma partição finita do intervalo $[0,1]$, chamada partição de Yoccoz real de nivel $n$, cujos elementos são as componentes conexas 
de $[0,1] \backslash P_{n}$ e são chamados de peças de Yoccoz real de nivel $n$. Se $x \notin \cup_{i=-1}^{\infty} P_{i}$ existe uma única peça de Yoccoz real de nivel $n$ que contém $x$ a qual denotamos por $P_{n}(x)$. Pode-se verificar que $f\left(P_{n}(x)\right) \subseteq P_{n-1}(f(x))$ e $f\left(\partial P_{n}(x)\right) \subset \partial P_{n-1}(f(x))$. As peças de Yoccoz que contêm os pontos críticos, digamos $P_{n}(c)$ e $P_{n}(d)$, são de especial interesse. Em geral, para $x=c$, $d$ ocorre que $f_{\mid P_{n}(x)}$ é uma aplicação unimodal de $P_{n}(x) \mathrm{em}$ um subconjunto próprio de $P_{n-1}(f(x))$. Podemos ter portanto que $f\left(P_{n}(x)\right) \cap P_{n-1}(f(x)) \neq \emptyset$ sem que $f\left(P_{n}(x)\right)$ contenha $P_{n-1}(f(x))$. Sendo assim as partições de Yoccoz reais nào são em geral partições de Markov. No caso em que $P_{n}(x)$ não contém ponto crítico temos que $f_{\mid P_{n}(x)}$ é um difeomorfismo de $P_{n}(x)$ sobre $P_{n-1}(f(x))$.

Supondo que as órbitas positivas dos pontos críticos $c, d$ sào infinitas existem $P_{0}(c) \supset$ $P_{1}(c) \supset P_{2}(c) \supset \ldots$ e $P_{0}(d) \supset P_{1}(d) \supset P_{2}(d) \supset \ldots$ Quando $\cap_{i=0}^{\infty} P_{i}(c)$ é um intervalo não-degenerado dizemos que f́é renormalizável em c. Podemos definir de modo análogo o caso em que $f$ é renormalizável em $d$. Nós estaremos especialmente interessados no caso que chamamos de não-renormalizável, ou seja, o caso em que $f$ não é renormalizável em $c$ e $d$. No caso em que $c$ e $d$ são recorrentes temos interesse especial nas subsequêrıcias $P_{0}(c) \supset P_{m_{1}}(c) \supset P_{m_{2}}(x) \supset \ldots$ e $P_{0}(d) \supset P_{n_{1}}(d) \supset P_{n_{2}}(d) \supset \ldots$ tais que $m_{h+\mathrm{i}}-m_{h_{i}}$ e $n_{k+1}-n_{k}$ são, respectivamente, os tempos de primeiro retorno de $c$ e $d$ a $P_{m k_{k}}(c) \cup$ $P_{n_{k}}(d)$. Aqui consideramos $m_{0}=n_{0}=0 \mathrm{e} k \geq 0$. Dito de outra forma temos que $P_{m_{k+1}}(c) \cup P_{n_{k+1}}(d)$ é parte do domínio da aplicação de primeiro retorno a $P_{m_{k}}(c) \cup P_{n_{k}}(d)$ e $f^{m_{k+1}-m_{k}}\left(\partial P_{m_{k+1}}(c)\right) \cup f^{n_{k+1}-n_{k}}\left(\partial P_{n_{k+1}}(d)\right) \subset \partial\left(P_{m_{k}}(c) \cup P_{n_{k}}(d)\right)$. Portanto, se $I_{k}$ e $J_{k}$ sào as sequências de intervalos definidas na introdução temos que $I_{k}=P_{m_{k}}(c)$ e $J_{k}=P_{n_{k}}(d)$. Também temos que $\phi_{k}(c)=f^{m_{k+1}-m_{k}}(c)$ e $\phi_{k}(d)=f^{n_{k+1}-n_{k}}(c)$. Em geral as sequências de tempos $m_{k+1}-m_{k}$ e $n_{k+1}-n_{k}$ são completamente independentes, mas no caso em que estaremos mais interessados, o caso em que elas são as menores possíveis dentro de 
uma certa classe de polinômios, elas serão simultaneamente a sequência de Fibonacci $2,3,5,8,13, \ldots$. Observamos ainda que cada componente do domínio de $\phi_{k}$ é uma peça de Yoccoz real.

\subsection{Polinômios Cúbicos Fibonacci}

Consideramos polinômios cúbicos $f:[0,1] \rightarrow[0,1]$, definidos por $f(x)=P_{a b}(x)=$ $a x^{3}+b x^{2}+(1-a-b) x$ ou $f(x)=Q_{a b}(x)=1-a x^{3}-b x^{2}-(1-a-b) x$, onde os parâmetros $a, b \in \mathbb{R}$ são escolhidos de forma que o polinômio $f$ possui dois extremos locais em $(0,1)$ os quais denotamos por $c$ e $d$. Então, o intervalo $[0,1]$ possui uma partição com três intervalos $[0, c],[c, d]$ e $[d, 1]$, restrito a cada qual $f$ é estritamente monótona. Estes pontos $c$ e $d$ são pontos críticos de $f$.

Assumimos que $f$ é não-renormalizável e que $c$ e $d$ são recorrentes, não-periódicos e tais que $\omega(c)=\omega(d)$. Sejam então $I_{0} \supset I_{1} \supset I_{2} \supset \ldots \supset\{c\}$ e $J_{0} \supset J_{1} \supset J_{2} \supset \ldots \supset\{d\}$ tais que $I_{n}$ e $J_{n}$ são componentes do domínio da aplicação de primeiro retorno $\phi_{n}$ a $I_{n-1} \cup J_{n-1}$. Para efeitos de uniformidade da notação convencionaremos que $\phi_{0}=f$. As restriçôes de $\phi_{n \mid I_{n}}$ e $\phi_{n \mid J_{n}}$ são chamados ramos críticos. Estes ramos são apicações unimodais cujas imagens estão contidas em $I_{n-1}$ ou $J_{n-1}$. Todos os outros ramos de $\phi_{n}$ não possuem pontos críticos e aplicam seus domínios difeomorficamente sobre $I_{n-1}$ ou $J_{n-1}$. Se $\phi_{n}(c) \in I_{n} \cup J_{n}$ ou $\phi_{n}(d) \in I_{n} \cup J_{n}$ dizemos que $f$ possui um retorno central.

Se $f$ não possui retornos centrais então existem duas componentes conexas (possivelmente idênticas) do domínio de $\phi_{n}$ denotadas por $C_{n}$ e $D_{n}$ as quais satisfazem $\phi_{n}(c) \in C_{n}$, $\phi_{n}(d) \in D_{n}$ e os ramos $\phi_{n \mid C_{n}}, \quad \phi_{n \mid D_{n}}$ são difeomorfismos. Chamamos $C_{n}$ e $D_{n}$ de domínios pós-criticos. 
Definição 2.2.1 Se $f$ não possui retornos centrais e existe um inteiro positivo $n_{0} \geq 1$ tal que para todo $n \geq n_{0} \quad \phi_{n \mid C_{n}}=\phi_{n-1 \mid C_{n}}, \quad \phi_{n \mid D_{n}}=\phi_{n-1 \mid D_{n}}, \quad \phi_{n+1}(c)=\phi_{n \mid C_{n}} \circ \phi_{n \mid I_{n}}(c)$ $\epsilon \phi_{n+1}(d)=\phi_{n \mid D_{n}} \circ \phi_{n \mid J_{n}}(d)$ dizemos que $f$ é eventualmente Fibonacci. Quando $n_{0}=1$ dizemos que $f$ é Fibonacci.

Observe que um polinômio cúbico Fibonacci não possui retornos centrais mas os seus domínios pós-críticos $C_{n}$ e $D_{n}$ săo tais que $C_{n} \cup D_{n} \subset I_{n-1} \cup J_{n-1}$ e para $x=c, d$ verifica-se que $\phi_{n+1}(x)=\phi_{n-1} \circ \phi_{n}(x)$. Isto quer dizer que os tempos de retomo de polinômios cúbicos Fibonacci, como provaremos mais adiante, sào os menores possíveis se considerarmos apenas os polinômios sem retornos centrais. Isto garante uma forte recorrência e entào acreditamos que se os polinômios Fibonacci exibem decaimento de geometria, o que de fato é um dos nossos principais resultados, todos os polinômios com retornos näo-centrais também devem ter esta mesma propriedade. O conceito de polinômio cúbico eventualmente Fibonacci é bastante natural uma vez que propriedades como o decaimento de geometria e outras não dependem do comportamento de um número finito de aplicações $\phi_{n}$.

Os polinômios do tipo eventualmente Fibonacci exibem alguns tipos combinatórios específicos os quais procuraremos identificar. Estes tipos podem ser identificados em termos das posições dos domínios pós-críticos $C_{n}, D_{n}$ e de suas imagens pelo primeiro retorno $\phi_{n}$ a $I_{n-1} \cup J_{n-1}$. Consideramos as seguintes possibilidades (veja as Figuras 2.1 e 2.2):

- Tipo $F_{1}: C_{n} \cup D_{n} \subset I_{n-1}$ e $\phi_{n}\left(C_{n} \cup D_{n}\right)=J_{n-1}$.

- Tipo $F_{2}: C_{n} \cup D_{n} \subset J_{n-1}$ e $\phi_{n}\left(C_{n} \cup D_{n}\right)=I_{n-1}$.

- 'Tipo $F_{3}: C_{n} \subset I_{n-1}, \quad D_{n} \subset J_{n-1}$ e $\phi_{n}\left(C_{n}\right)=J_{n-1}, \phi_{n}\left(D_{n}\right)=I_{n-1}$. 
- Tipo $F_{4}: C_{n} \subset J_{n-1}, \quad D_{n} \subset I_{n-1}$ e $\phi_{n}\left(C_{n}\right)=J_{n-1}, \quad \phi_{n}\left(D_{n}\right)=I_{n-1}$.

- Tipo $F_{5}: C_{n} \subset J_{n-1}, \quad D_{n} \subset I_{n-1} \quad$ e $\quad \phi_{n}\left(C_{n}\right)=I_{n-1}, \quad \phi_{n}\left(D_{n}\right)=J_{n-1}$.
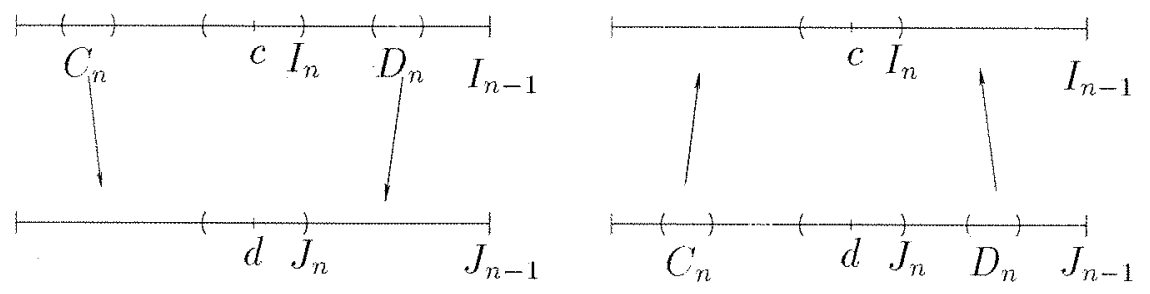

Figura 2.1: Fibonacci Tipo $F_{1}$ e $F_{2}$

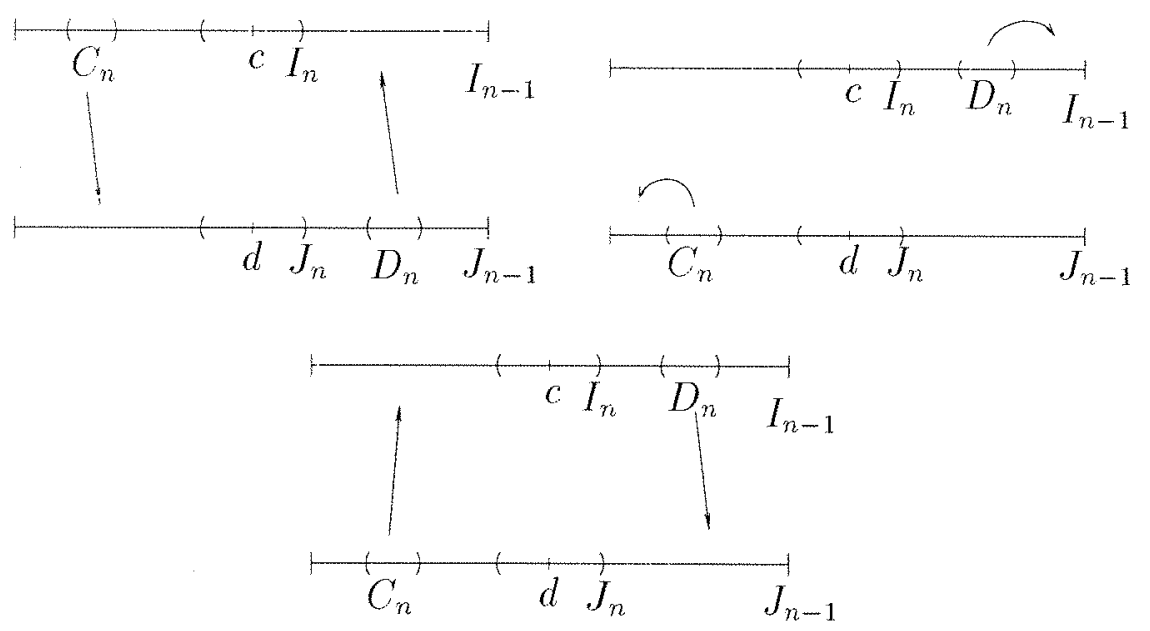

Figura 2.2: Fibonacci Tipo $F_{3}, F_{4} \in F_{5}$ 


\subsection{Resultados Principais}

Inicialmente provaremos que de fato existem polinômios cúbicos Fibonacci, este é o conteúdo do teorema seguinte:

Teorema 2.3.1 As famílias $P_{a b}$ e $Q_{a b}$ possuem polinômios cúbicos Fibonacci cujos pontos críticos são recorrentes e possuem o mesmo w-limite.

A partir do Teorema 2.3.1 podemos concluir que existem muitos polinômios cúbicos eventualmente Fibonacci cujos pontos críticos são recorrentes e possuem o mesmo $\omega$ limite. No entanto exibiremos mais adiante as sequências de kneading (etinerários dos valores críticos $f(c)$ e $f(d))$ dos polinômios cúbicos Fibonacci e disto decorrerá que todos eles estão em uma única classe de conjugação topológica.

As sequências kneading e a classe de conjugação topológica de um polinômio podem ser determinadas a partir de uma sequência especial de aplicações de primeiro retorno associada ao polinômio. O teorema a seguir descreve propriedades das aplicações desta sequência.

Teorema 2.3.2 Seja $f=P_{a b}$ ou $f=Q_{a b}$ um polinômio cúbico eventualmente Fibonacci cujos pontos críticos são recorrentes e possuem o mesmo w-limite. Entâo existe n tal que a seqüência $\phi_{n}, \phi_{n+1}, \phi_{n+2}, \ldots$ exibe a seqüência de tipos $F_{1} F_{2} F_{1} F_{2} \ldots$ ou $F_{3} F_{4} F_{5} F_{3} F_{4} F_{5} \ldots$

Dado um polinômio cúbico $f$ com pontos críticos recorrentes consideramos as sequências $I_{0} \supset I_{1} \supset I_{2} \supset \ldots$ e $J_{0} \supset J_{1} \supset J_{2} \supset \ldots$ de intervalos que definimos anteriormente. Para cada $x \in[0,1]$ e $n \geq 1$ consideramos o menor $\tau_{n}(x) \in \mathbb{N}^{*} \cup\{\infty\}$ tal que $f^{\tau_{n}(x)}(x) \in I_{n-1} \cup J_{n-1}$. Este $\tau_{n}(x)$ é chamado tempo de primeira entrada em $I_{n-1} \cup J_{n-1}$. 
Sejam então a sequência de tempos $\tau(x)=\left(\tau_{1}(x), \tau_{2}(x), \ldots\right)$. Quando for necessário usaremos a notação $\tau_{n}(x, f)$ e $\tau(x, f)$ para indicar a dependência em $f$ também. Estamos especialmente interessados nas seqüencias de tempos dos pontos críticos $c$ e $d$. Observe que $\phi_{n}(c)=f^{\tau_{n}(c)}(c)$ e $\phi_{n}(d)=f^{\tau_{n}(d)}(d)$. Em particular denotaremos por $s_{n}=\tau_{n}(c)$ e $r_{n}=\tau_{n}(d)$. Como $\phi_{0}:=f$, definimos $s_{0}=r_{0}=1$ No Teorema 2.3.4 provaremos que as sequências de tempos $s_{n}$ e $r_{n}$ associadas a um polinômio cúbico Fibonacci são ambas idênticas à sequência de Fibonacci $\alpha=\left(2,3,5, \ldots, \alpha_{n}, \ldots\right)$, onde $\alpha_{1}=2, \alpha_{2}=3$ e para $n \geq 2$ tem-se que $\alpha_{n+1}=\alpha_{n}+\alpha_{n-1}$.

Consideramos o espaço das sequências infinitas

$$
\Sigma^{\mathbb{N}}=\left\{t=\left(t_{1}, t_{2}, \ldots\right): t_{i} \in \mathbb{N} \text { para todo } i \geq 1\right\}
$$

munido da ordem lexicográfica que denotamos por $\prec$. Então se $s, t \in \Sigma^{\mathbb{N}}$ são tais que $s \prec t$ dizemos $s$ é menor do que $t$. Em $\Sigma^{\mathbb{N}}$ existem algumas sequências, as quais chamamos de sequências admissíveis, que podem ser realizadas como sequência de tempos de um polinômio cúbico. Gostaríamos de saber se existe um polinômio cúbico $f$ cujas sequências de tempos $\tau(c)$ e $\tau(d)$ sejam, simultaneamente, a sequència menor possivel dentre as admissíveis.

Teorema 2.3.3 Se fé um polinômio cúbico do tipo eventualmente Fibonacci cujos pontos críticos são recorrentes e possuem o mesmo $\omega$-limite. Entâo $s_{n+1}=s_{n}+s_{n-1}+s e$ $r_{n+1}=r_{n}+r_{n-1}+r$ com $|s|,|r| \leq\left|s_{1}-r_{1}\right|+\left|s_{0}-r_{0}\right|$. Em particular, se $s_{0}=r_{0}=1 \mathrm{e}$ $s_{1}=r_{1}=2$ entâo $s_{n}$ e $r_{n}$ são iguais a sequência de Fibonacci.

O próximo teorema nos garante que dentre as sequências realizadas por polinômios que não possuem retornos centrais a sequência de Fibonacci é a menor possivel e é 
realizada pelos polinômios Fibonacci justificando assim esta nomeclatura.

Teorema 2.3.4 1. Se $f=P_{a b}$ ou $f=Q_{a b}$ é um polinômio cúbico Fibonacci cujos pontos críticos são recorrentes e possuem o mesmo $\omega$-limite então verifica-se que ambas as sequências $\tau(c, f)$ e $\tau(d, f)$ coincidem com a sequência de Fibonacci.

2. Se $g=P_{a b}$ ou $g=Q_{a b}$ é um polinômio cúbico tal que $\tau(c, g)$ ou $\tau(d, g)$ é menor do que a sequência de Fibonacci entäo g possui retornos centrais.

Como vimos no Teorema 2.3.4, dentre os polinômios cúbicos que não apresentam retornos centrais, os polinômios Fibonacci são aqueles cujos pontos críticos possuem os menores tempos de retorno determinando assim uma forte recorrência dos mesmos. Isto mostra que os polinômios Fibonacci formam uma classe de especial importância na qual o decaimento de geometria é uma questão delicada. Esperamos que a partir do decaimento de geometria que obtivemos no teorema a seguir para os polinômios Fibonacci (e mais geralmente para os polinômios eventualmente Fibonacci) poderemos obter esta mesma propriedade para todos os polinômios que não apresentam retornos centrais ou mesmo para os que exibam cascatas limitadas de retornos centrais.

Teorema 2.3.5 Se $f=P_{a b}$ ou $f=Q_{a b}$ é um polinômio cúbico eventualmente Fibonacci cujos pontos críticos são recorrentes e possuem o mesmo w-limite entäo f exibe decaimento exponencial de geometria.

A presença de pontos críticos para um polinômio $f$ contribui para a complexidade da sua dinâmica. No intuito de obter algumas informações podemos tentar eliminar estes pontos críticos definindo uma outra aplicação que em certos intervalos coincide 
com iteradas de $f$. Isto nos leva ao conceito de aplicaçâo de Markov topológica induzida por $f$ que introduzimos abaixo.

Definição 2.3.1 Seja $P=\left\{N_{j}\right\}_{j=1}^{\kappa}(\kappa \leq \infty)$ uma coleção de intervalos $N_{j} \subset \mathbb{R}$ nãodegenerados e cujos interiores são dois a dois disjuntos. Uma aplicaçấo $F: \cup_{j=1}^{\kappa} N_{j} \rightarrow \mathbb{R}$ é chamada de aplicação de Markov se cada ramo $F_{\mid N}$ é um homeomorfismo sobre a sua imagem a qual contém todos os intervalos $N_{i}$ tais que o interior de $N_{i} \cap F\left(N_{j}\right)$ é nãovazio. Dizemos que $F \dot{e}$ de classe $C^{r}(r \geq 0)$ se cada ramo $F_{\mid N_{j}}$ possui uma txtensão para um difeomorfismo de classe $C^{r}$ definido em um intervalo aberto que contém o fecho de $N_{j}$. A coleção de intervalos $F(\mathcal{P})=\left\{F\left(N_{j}\right)\right\}_{j=1}^{\kappa}$ é chamada de partição imagem. Se cada ramo $F_{N_{j}}$ coincide com um iterado $f_{\mid N_{j}}^{n_{j}}$, para algum $n_{j}$, dizemos que $F$ é uma aplicação de Markov induzida por $f$.

Uma aplicação de Markov induzida por $f$ com propriedades apenas topológicas não é suficiente para garantir boas propriedades métricas e portanto introduzimos mais um conceito.

Definição 2.3.2 Uma aplicação de Markov $F: \cup_{j=1}^{\kappa} N_{j} \rightarrow \mathbb{R}$ de classe $C^{r}(r \geq 1)$ é dita hiperbólica em $[0,1]$ se:

1. O domínio de $F$ é uma união de intervalos abertos $\cup_{j=1}^{\kappa} N_{j}$ com medida de Lebesgue total em $[0,1]$.

2. A partiçâo imagem $F(\mathcal{P})=\left\{F\left(N_{j}\right)\right\}_{j=1}^{\kappa}$ é uma coleção finita de intervalos limitados.

3. Existe $a>1$ tal que $|D F(x)| \geq a$, para todo $x \in \cup_{j=1}^{n} N_{j}$. 
4. Existe $K<\infty$ tal que $\frac{D F(x)}{D F(y)} \leq K$, para todo $x, y \in N_{j}$.

Dizemos que uma aplicação multimodal $f:[0,1] \rightarrow[0,1]$ de classe $C^{1}$ induz expansão ou induz hiperbolicidade se a mesma induz uma aplicação de Markov hiperbólica em $[0,1]$. Examinamos então a hiperbolicidde induzida nos polinômios cúbicos que não possuem retornos centrais e em particular no caso dos polinômios eventualmente Fibonacci. No caso eventualmente Fibonacci será necessário a propriedade do decaimento exponencial de geometria, mas no caso básico, veja a difinição mais adiante, mostraremos a. hiperbolicidade induzida sem usar essa propriedade.

Teorema 2.3.6 Todo polinômio cúbico real da família $P_{a b}$ ou $Q_{a b}$ que não possui retornos centrais e exibe decaimento exponencial de geometria induz hiperbolicidade.

Um aspecto importante para a compreensão de um sistema dinâmicó é descriçâo dos seus atratores os quais podem ser topológicos ou métricos. Dado um conjunto compacto $\mathcal{A}$ positivamente invariante por $f($ isto é $f(\mathcal{A}) \subset \mathcal{A}$ ) definimos a sua bacia de atracáo por

$$
B(\mathcal{A})=\{x \in[0,1] \text { tal que } \omega(x) \subset \mathcal{A}\}
$$

O conjunto $\mathcal{A}$ é chamado de atrator topológico se

- $B(\mathcal{A})$ contém um conjunto de segunda categoria de Baire;

- Se existe um conjunto positivamente invariante $\mathcal{A}^{\prime}$ estritamente contido em $\mathcal{A}$, então $\overline{B(\mathcal{A})} \backslash \overline{B\left(\mathcal{A}^{\prime}\right)}$ é um conjunto de segunda categoria de Baire. 
Sabemos que se $f$ é não-renormalizável e não possui atrator periódico então o seu atrator topológico é um intervalo invariante no qual $f$ é transitiva, veja [L1] e [MS], página 236.

O conjunto $\mathcal{A}$ é chamado de atrator métrico se

- A bacia de atração $B(\mathcal{A})$ tem medida de Lebesgue positiva;

- Se $\mathcal{A}^{\prime}$ é um conjunto positivamente invariante e estritamente contido em $\mathcal{A}$, então $B(\mathcal{A}) \backslash \mathcal{B}\left(\mathcal{A}^{\prime}\right)$ tem medida positiva.

Em [L1] e [SV2]) encontramos uma descrição dos atratores métricos (que existem por $[M]$ ) de aplicações multimodais de classe $C^{2}$. Segundo esta descrição um polinômio cúbico eventualmente Fibonacci possui lim único atrator métrico que poderia ser um ciclo de intervalos transitivos ou um conjunto de Cantor minimal contendo os pontos críticos $c$ e $d$. Uma vez que o atrator topológico de tais polinòmios é deste primeiro tipo chamamos este último tipo, caso seja o atrator métrico, de airator selvagem.

Em geral, se $\mathcal{A}$ é um atrator métrico que não é um atrator topológico então será chamado de atrator selvagem. A questão da existência ou não de atratores selvagens foi colocada por Milnor [M] e tem atraído a atenção de muitos pesquisadores. No caso de polinômios quadráticos, o que possui a combinatória de Fibonacci foi considerado o principal candidato a exibir um atrator selvagem, veja $[\mathrm{HK}]$ (veja também $[\mathrm{LM}]$ ).

Como foi provado em [LM] isto de fato não ocorre. No entanto o conjunto w-limite $\omega(c)$ para um polinômio unimodal real com ponto crítico suficientemente degenerado (a saber $x \mapsto x^{2 l}+a \operatorname{com} l$ suficientemente grande) exibindo a combinatória de Fibonacci é um atrator selvagem, veja [BKNV]. 
O Teorema 2.3.6 garante a existência de uma aplicação de Markov hiperbólica $F$ induzida por $f$ e que pelo teorema do folclore possui uma p.i.a.c. ergódica que denotamos por $\mu$. Isto é suficiente para garantir que para $x$ q.t.p. (em relaçào à medida de Lebesgue) a órbita positiva $\operatorname{orb}_{f}^{+}(x)$ é densa em um intervalo. Com isto podemos concluir que um polinômio cúbico Fibonacci não possuem atratores selvagens. Na verdade qualquer polinômio cúbico com retornos não-centrais e decaimento de geometria não possuem atratores selvagens. 


\section{Capítulo 3}

\section{Ferramentas Básicas}

\subsection{Princípios de Koebe e Outros Fatos}

No estudo das propriedades métricas do sistema dinâmico gerado pelas iteraçòes de uma função multimodal $g$ é indispensável que se tenham estimativas da distorçào ou da não-linearidade das iteradas $g^{n} \mathrm{em}$ certos intervalos. Nesta seção introduzimos brevemente este conceito e importantes ferramentas que nos permitem ter estas estimativas.

Seja $g$ é uma aplicação de classe $C^{3}$. Definimos a sua derivada de Schwarz por

$$
S g(x)=\frac{g^{\prime \prime \prime}(x)}{g^{\prime}(x)}-\frac{3}{2}\left(\frac{g^{\prime \prime}(x)}{g^{\prime}(x)}\right)^{2},
$$

se $g^{\prime}(x) \neq 0$. Se $g^{\prime}(x)=0$ definimos $S g(x)=-\infty$. Dizemos que $g$ tem derivada de Schwarz negativa e denotamos por $S g<0$ se $S g(x)<0$ para todo $x$.

Se $g$ é um polinômio com coeficientes reais cujas raízes são todas reais e distintas então verifica-se que $S g<0$. A classe das aplicações multimodais de classe $C^{3}$ e com derivada de Schwarz negativa é um conjunto aberto na topologia $C^{3}$. Sabe-se também 
que a composição de duas aplicações com derivada de Schwarz negativa resulta em uma aplicaçào com derivada de Schwarz negativa. Este fato faz da classe das aplicações com derivada de Schwarz negativa uma classe de particular interesse em sistemas dinâmicos, visto que as iteradas da aplicações com derivada de Schwarz negativa também têm esta mesma propriedade.

Singer provou em [S] que se $g$ possui derivada de Schwarz negativa então todo ponto periódico atrator de $g$ atrai $\mathrm{um}$ ponto crítico ou um dos extremos do seu domínio. Os polinômios $f$ que estamos considerando possuem derivada de Schwarz negativa e como estamos assumindo que os pontos críticos sào recorrentes e não-periódicos e que os extremos do intervalo $[0,1]$ são pontos fixos repulsores, temos que $f$ não possui pontos periódicos atratores.

O conceito de distorçào que introduziremos a seguir desempenha um papel central na análise de propriedades métricas.

Definição 3.1.1 Seja g um difeomorfismo definido no intervalo J. Definimos a distorção de $g$ em $J$, denotada por dist $(g, J)$, do seguinte modo:

$$
\operatorname{dist}(g, J):=\sup \left\{\frac{|D g(x)|}{|D g(y)|}, \quad x, y \in J\right\}
$$

A quantidade $\operatorname{dist}(g, J)$ é uma medida de quanto a aplicação $g$ distorce a razão de dois intervalos adjacentes $J_{1}, J_{2}$ contidos em $J$.

A derivada de Schwarz também se tornou uma ferramenta útil para o controle de distorção de iteradas de aplicaçoes do intervalo. Os principais fatos nesta direção são os princípios de Koebe enunciados no lema abaixo cuja prova pode ser encontrada em [MS]. Para enunciá-los introduzimos alguns conceitos. Denotaremos por $|A|$ a medida de Lebesgue de um Boreleano $A$. Se $\sigma>0$ e $U \subset V$ são intervalos tais que para 
cada componente conexa $L$ de $V \backslash U$ verifica-se que $|L| \geq \sigma|U|$ dizemos que $V$ é uma $\sigma$-vizinhança de $U$ ou que $U$ está $\sigma$-centralizado em $V$.

Lema 3.1.1 Sejam $J \subset T$ intervalos e $g$ difeomorfismo definido $\mathrm{em} T$. Se g tem derivada de Schwarz negativa e $g(T)$ é uma $\delta$-vizinhança de $g(J)$, então verifica-se que:

1. Existe $\alpha>0$ tal que $T$ é uma $\alpha$-vizinhança de $J$ e a depende apenas de $\delta$

2. $\operatorname{dist}(g, J) \leq\left(\frac{1+\delta}{\delta}\right)^{2}$.

Prova: Veja [MS], páginas 267-302.

Devido ao Lema 3.1.1 os conceitos de extendibilidade a seguir surgem relacionados com o conceito de distorção.

Definição 3.1.2 Uma aplicaçâo real contínua h definida em um intervalo fechado $U$ é dita unimodal se $h(\partial U)$ é um único ponto e existe um ponto $x$ no interior de $U$ tal que $h$ é estritamente monótona em cada uma das componentes de $U \backslash\{x\}$.

Definição 3.1.3 - Sejam $V$ um intervalo e $F=f^{k}$ um difeomorfismo de $V$ sobre $F(V)$. Se existe um intervalo $\widehat{V} \supset V$ restrito ao qual $f^{k}$ é um difeomorfismo $e$ $f^{k}(\widehat{V})$ é uma $\alpha$-vizinhança de $f^{k}(V)$ dizemos que $F_{\mid V}$ é $\alpha$-extensivel. Neste caso denotamos $f_{\mid \hat{V}}^{k}$ por $\widehat{F}$.

- Suponhamos que $f$ e $f^{k+1}$ são aplicaçôes unimodais definidas em um intervalo $U$ e que $f^{k}$ é um difeomorfismo definido em um intervalo $V$ tal que $f(U) \subset V$. Entäo, se existem intervalos $\widehat{U} \supset U \in \widehat{V} \supset V$ tais que $f^{-1}(\partial \widehat{V}) \subset \widehat{U}, f_{\hat{U}} \dot{e}$ unimodal $e$ 
$f_{\mid \widehat{V}}^{k}$ é um difeomorfismo cuja imagem é uma a-vizinhança de $f^{k}(V)$ dizemos que a aplicação unimodal $h=f_{\mid U}^{k+1}$ é $\alpha$-extensível. Neste caso denotamos $f_{\mid \hat{U}}^{k+1}$ por $\widehat{h}$. 


\subsection{Ferramentas de análise complexa}

\subsubsection{Anéis e módulos de anéis}

Para provar decaimento exponencial de geometria, vamos utilizar extensões de $\phi_{n}$ para vizinhanças adequadas no plano complexo. Cada componente conexa do domínio de $\phi_{n}$ na reta real se estende a um disco topológico contido no plano complexo. Em particular os ramos críticos $\phi_{n \mid I_{n}}$ e $\phi_{n \mid J_{n}}$ se estendem, respectivamente, para ramos holomorfos definidos em discos topológicos $\mathcal{I}_{n}$ e $\mathcal{J}_{n}$ contidos no plano complexo. Entào teremos duas sequências encaixantes $\mathcal{I}_{0} \supset \mathcal{I}_{1} \supset \ldots$ e $\mathcal{J}_{0} \supset \mathcal{J}_{1} \supset \ldots$ de discos topológicos. Para provarmos decaimento de geometria devemos estudar as sequências dos tamanhos dos anéis topológicos $\mathcal{I}_{n} \backslash \overline{\mathcal{I}_{n+1}}$ e $\mathcal{J}_{n} \backslash \overline{\mathcal{J}_{n+1}}$. O conceito de módulo de um anel topológico que introduziremos a seguir nos permitirá medir estes tamanhos.

Seja $A(d)=\{z \in \mathbb{C}: 0<d<|z|<1\}$. Dizemos que um conjunto aberto $\mathbb{A} \subset \mathbb{C}$ é um anel topológico se ele for homeomorfo a $A(d)$, para algum $d>0$. De acordo com a clássica teoria de classificação dos abertos do plano complexo sabemos que para todo anel topológico $\mathbb{A} \subset \mathbb{C}$ existe uma aplicação conforme com inversa conforme (chamada aplicação de Riemann) que o aplica sobre um anel $A(d)$, onde $d>0$ é único. Então definimos o módulo de $\mathbb{A}$, que denotaremos por $\bmod (\mathbb{A})$, como sendo o número - $\log d$. O módulo de um anel é um invariante conforme. Sejam $\mathbb{A}$ e $\mathbb{A}^{\prime}$ anéis e $f: \mathbb{A} \rightarrow \mathbb{A}^{\prime}$ um recobrimento holomorfo de grau $k$. Então $\bmod \left(\mathbb{A}^{\prime}\right)=k \cdot \bmod (\mathbb{A})$.

Sejam $\mathbb{A}$ e $\mathbb{A}^{\prime}$ anéis disjuntos tais que um deles está contido na componente conexa limitada do complementar do outro. Então definimos uma operação comutativa $\oplus$ que a cada par de anéis $\mathbb{A}$ e $\mathbb{A}^{\prime}$ com a propriedade acima associa o menor anel $\mathbb{A} \oplus \mathbb{A}^{\prime}$ que 
contem $\mathbb{A}$ e $\mathbb{A}^{\prime}$. Esta operação possui uma propriedade importante na estimativa de módulos de anéis, chamada super-aditividade, isto é:

$$
\bmod \left(\mathbb{A} \oplus \mathbb{A}^{\prime}\right) \geq \bmod (\mathbb{A})+\bmod \left(\mathbb{A}^{\prime}\right)
$$

Veja a prova desta propriedade em [LV].

Para provarmos decaimento de geometria é necessário provarmos o crescimento linear de $\bmod \left(\mathcal{I}_{n} \backslash \overline{\mathcal{I}_{n+1}}\right)$ e $\bmod \left(\mathcal{J}_{n} \backslash \overline{\mathcal{J}_{n+1}}\right)$. A nossa estratégia é a de provar inicialmente que estas sequências de módulos não decrescem. Então para provarmos o crescimento linear que necessitamos lançaremos mão do lema seguinte que é uma importante ferramenta.

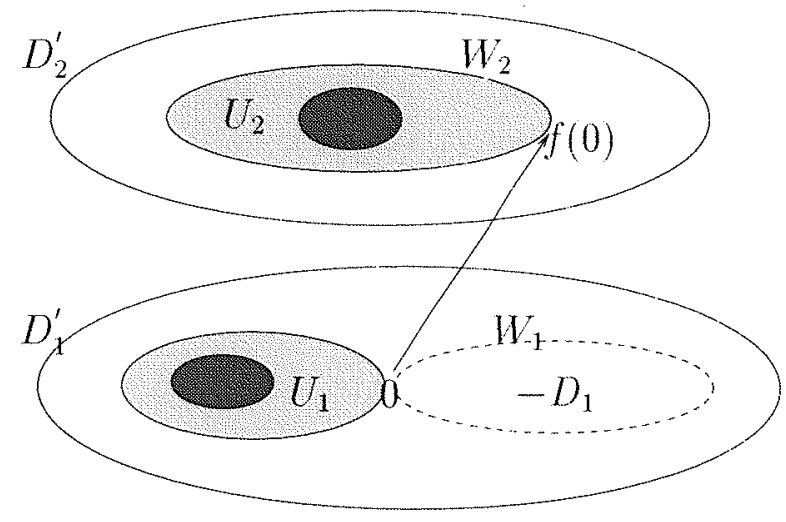

Lema 3.2.1 Considere um disco topológico $D_{1}^{\prime}$, um anel $U_{1} \subset D_{1}^{\prime}$ e um disco topológico $D_{1}$ determinado por uniäo de $U_{1}$ junto com a componente conexa limitada do seu complementar. Denote $W_{1}=D_{1}^{\prime} \backslash \overline{D_{1}}$. Considere uma aplicação holomorfa f na forma de $h\left(z^{2}\right)$ com h univalente, assuma que $f$ é univalente em $D_{1}$. Defina $D_{2}:=f\left(D_{1}\right), W_{2}:=D_{2}^{\prime} \backslash \overline{D_{2}}$ e $U_{2}:=f\left(U_{1}\right)$. Escolha números não negativos $\sigma_{1}$ e $\sigma_{2}$ tais que

$$
\sigma_{2} \leq \bmod U_{2} \text { e } \sigma_{1} \leq \bmod U_{2}+\bmod W_{2}
$$




\section{Então}

1. $\bmod \left(U_{1}\right)+\bmod \left(W_{1}\right) \geq \frac{1}{2}\left(\sigma_{1}+\sigma_{2}\right)$

2. Para todo $\delta>0$ existe um $\epsilon>0$ tal que, se $\sigma_{1}-\sigma_{2} \geq \delta$ e $\bmod \left(U_{2}\right) \geq \delta$ entâo

$$
\bmod \left(U_{1} \oplus W_{1}\right) \geq \frac{1}{2}\left(\sigma_{1}+\sigma_{2}\right)+\epsilon
$$

Prova: Veja a prova em [GS3]. 


\subsubsection{Partição de Yoccoz complexa}

Supomos como antes que $f=P_{a b}$ ou $f=Q_{a b}$, onde $a$ e $b$ são parâmetros reais tais que $f$ possui dois pontos críticos recorrentes $c$ e $d$ com o mesmo $\omega$-limite. Assumimos também que $f$ não possui retornos centrais, em particular $f$ é não-renormalizável.

As aplicações de primeiro retorno $\phi_{n}$ podem ser extendidas para aplicações conformes que agem no plano complexo. Os elementos do que chamaremos de partiçâo de Yoccoz complexa surgem naturalmente como domínios de ramos destas extensões.

Consideremos certos aspectos da dinâmica de $f$ na esfera de Riemann $\mathbb{C} \cup\{\infty\}$. Sabemos por $[B]$ que existem uma vizinhança $U$ de $\infty$ e um difeomorfismo conforme $h$ que conjuga $f_{\mid U} \operatorname{com} z \mapsto z^{3}$ em $V:=\{z \in \mathbb{C}:|z|<1 / 2\}$. Em particular $f(\bar{U}) \subset U$ e $\cup_{i=0}^{\infty} f^{-i}(U)$ coincide com a bacia de atração de $\infty$ que denotamos por $W^{s}(\infty)$ e está definida por

$$
W^{s}(\infty)=\{z \in \mathbb{C}: \omega(z)=\infty\}
$$

De fato podemos estender $h$ para uma conjugação conforme (que ainda denotamos por h) de $f_{\mid W^{s}(\infty)} \operatorname{com} z \mapsto z^{3}$ em $D:=\{z \in \mathbb{C}:|z|<1\}$.

Para $z \in D \backslash\{0\}$ existem únicos $\rho \in(0,1)$ e $\theta \in[0,2 \pi)$ tais que $z=\rho e^{i \theta}$. Como $z \mapsto z^{3}$ preserva a família dos círculos centrados na orígem e $\rho=$ constante e a família das semiretas a partir da origem e $\theta=$ constante temos que a imagem por $h^{-1}$ destes círculos (chamadas curvas equipotênciais) e semiretas (chamadas raios) são preservadas por $f$. Para cada ponto fixo $p$ de $f$ em $[0,1]$ existem pelo menos 2 raios $\alpha(p)$ e $\beta(p)$ tais que um é a imagem espelhada no eixo real do outro e $\overline{\alpha(p)} \backslash \alpha(p)=\overline{\beta(p)} \backslash \beta(p)=\{p, \infty\}$. Neste caso dizemos que $\alpha(p)$ e $\beta(p)$ aterrizam em $p$.

Para definirmos as partições de Yoccoz complexas consideramos $D_{0}$, um disco to- 
pológico em $\mathbb{C}$ limitado por uma curva equipotencial. Para cada $p \in P_{-1}$ (lembramos que $P_{-1}$ é o conjunto de pontos fixos de $f$ em $\left.[0,1]\right)$ existem 2 raios $\alpha(p)$ e $\beta(p)$ que aterrizam em $p$. Para cada $p^{*}$ tal que $f\left(p^{*}\right)=f(p)$ existem 2 raios que sào pré-imagens por $f$ dos raios $\alpha(p)$ e $\beta(p)$. Denotamos por $R_{0}$ o conjunto de todos os raios obtidos desta forma a partir de pontos fixos $p \in P_{-1}$. Então os raios de $R_{0}$ definem uma partição de $D_{0}$ que chamamos de partição de Yoccoz complexa de nível zero que denotamos por $\mathcal{P}_{0}$. Para cada $x \in D_{0}$ que não pertença a algum raio de $R_{0}$ temos o elemento $\mathcal{P}_{0}(x)$ desta partição, o qual chamamos de peşa de Yoccoz complexa de nível zero, que contem x. Agora podemos definir por indução as partiçōes de Yoccoz complexas de um nível qualquer. Assumimos definidos o disco topológico $D_{n}$, o conjunto de raios $R_{n}$ e a partição de Yoccoz complexa de nível $n$, denotada por $\mathcal{P}_{n}$. Então definimos $D_{n+1}:=f^{-1}\left(D_{n}\right)$ e $R_{n+1}:=f^{-1}\left(R_{n}\right)$. Os raios de $R_{n+1}$ determinam uma partição de $D_{n+1}$ que é por definição a partição de Yoccoz complexa de nível $n+1$ e que denotamos por $\mathcal{P}_{n+1}$.

O conjunto $K_{f}:=\cap_{i=0}^{\infty} D_{i}$ chama-se conjunto de Julia cheio e a sua fronteira $J_{f}:=$ $\partial K_{f}$ chama-se conjunto de Julia. Para cada $n \geq 0$ e $x \in K_{f} \backslash \cup_{i=0}^{\infty} R_{i}$ o elemento $\mathcal{P}_{n}(x)$ de $\mathcal{P}_{n}$ que contém $x$ está bem definido e chama-se peça de Yoccoz complexa de nível n.

Pode-se verificar que se $\mathcal{P}_{n}(x)$ não contém pontos críticos então $f$ é um difeomorfismo conforme de $\mathcal{P}_{n}(x)$ sobre $\mathcal{P}_{n-1}(f(x))$. Porém, no caso em que $\mathcal{P}_{n}(x)$ contém um ponto crítico, $f$ é um recobrimento conforme ramificado de grau 2 de $\mathcal{P}_{n}(x)$ sobre $\mathcal{P}_{n-1}(f(x))$ cujo ponto de ramificação é o ponto crítico.

Observe então que uma peça de Yoccoz real $P_{n}(x)$ é exatamente a interseção da peça de Yoccoz complexa $\mathcal{P}_{n}(x)$ com $\mathbb{R}$, em símbolos $P_{n}(x)=\mathbb{R} \cap \mathcal{P}_{n}(x)$. Isto nos permite associar a cada ramo de uma aplicação de primeiro retorno real $\phi_{k}$ a sua extensão para uma vizinhança no plano complexo. De fato, relembramos que $\phi_{k}$ é a aplicação de 
primeiro retorno a $I_{k-1} \cup J_{k-1}$ e temos que $I_{k-1}=P_{m_{k-1}}(c)$ e $J_{k-1}=P_{n_{k-1}}(d)$. Então cada componente do domínio de $\phi_{k}$ é uma peça de Yoccoz real $P_{j k}(x)$ que possui a sua correspondente peça de Yoccoz complexa $\mathcal{P}_{j_{k}}(x)$. Sendo assim, se $\phi_{k}=f^{l}$ em $P_{j_{k}}(x)$, definimos $\Phi_{k}:=f^{l} \mathrm{em} \mathcal{P}_{j_{k}}(x)$. Resulta que $\Phi_{k}$ é uma extensão de $\phi_{k}$ para o plano complexo.

Supondo que $c, d \in K_{f} \backslash \cup_{i=0}^{\infty} R_{i}$ temos que $\mathcal{P}_{0}(c) \supset \mathcal{P}_{1}(c) \supset \mathcal{P}_{2}(c) \supset \ldots$ e $\mathcal{P}_{0}(d) \supset \mathcal{P}_{1}(d) \supset \mathcal{P}_{2}(d) \supset \ldots$ Quando $f$ é não-renormalizável e $c, d$ são recorrentes, as subsequências $\mathcal{P}_{0}(c) \supset \mathcal{P}_{m_{1}}(c) \supset \mathcal{P}_{m_{2}}(x) \supset \ldots$ e $\mathcal{P}_{0}(d) \supset \mathcal{P}_{n_{1}}(d) \supset \mathcal{P}_{n_{2}}(d) \supset \ldots$ tais que $m_{k+1}-m_{k}$ e $n_{k+1}-n_{k}$ são, respectivamente, os tempos de primeiro retorno de $c$ e $d$ a $\mathcal{P}_{m_{k}}(c) \cup \mathcal{P}_{n_{k}}(d)$. Aqui consideramos $m_{0}=n_{0}=0$ e $k \geq 0$. Dito de outra forma temos que $\mathcal{P}_{m_{k+1}}(c) \cup \mathcal{P}_{n_{k+1}}(d)$ é parte do domínio da aplicação $\Phi_{k}$ que extende a aplicação de primeiro retorno $\phi_{k}$. Utilizemos a notaçào $\mathcal{I}_{k}=\mathcal{P}_{m_{k}}(c)$ e $\mathcal{J}_{k}=\mathcal{P}_{n_{k}}(d)$.

A aplicaçào $\Phi_{k}$ é uma continuação analítica do primeiro retorno $\phi_{k}$ e satisfaz às condições 2.-4. da Definição 3,2.1.

Definição 3.2.1 Uma aplicação holomorfa acoplada $\Phi$ é uma função analítica definida em um conjunto aberto $\mathcal{U} \subset \mathbb{C}$ que satisfaz as seguintes condiçôes:

1. $\mathcal{U}$ é uma uniâo finita ou infinita de discos topológicos abertos $\epsilon$ dois a dois disjuntos $\mathcal{U}_{i}$.

2. Existem discos topológicos disjuntos $\mathcal{I}$ e $\mathcal{J}$ tais que $\Phi$ aplica cada $\mathcal{U}_{i}$ sobre $\mathcal{I}$ ou $\mathcal{J}$.

3. $\Phi_{\mid \mathcal{U}_{i}}$ é univalente para $i \neq 0,1$ e para $i=0,1$ é um recobrimento ramificado de grau 2.

4. $\mathcal{U} \cap(\partial \mathcal{I} \cup \partial \mathcal{J})=\emptyset$. 


\section{Capítulo 4}

\section{Polinômios cúbicos Fibonacci e}

\section{eventualmente Fibonacci}

\subsection{Polinômios cúbicos Fibonacci}

Consideramos polinômios cúbicos $f:[0,1] \rightarrow[0,1]$, definidos por $f(x)=P_{a b}(x)=$ $a x^{3}+b x^{2}+(1-a-b) x \quad$ ou $\quad f(x)=Q_{a b}(x)=1-a x^{3}-b x^{2}-(1-a-b) x$, onde os parâmetros $a, b \in \mathbb{R}$. Nesta seção provaremos que existem parâmetros $a, b$ tais que $P_{a b}$ é Fibonacci. O mesmo pode ser provado para a família $Q_{a b}$.

Prova do Teorema 2.3.1. Para construir um polinômio $P_{a b}$ Fibonacci nós adotamos um procedimento análogo ao adotado na prova do Lema $1 \mathrm{em}$ [SV]. Nós começamos definindo por indução uma sequência $g_{n}$ de aplicações bimodais contínuas que converge para uma aplicação bimodal contínua $g$ que é Fibonacci.

Indução (primeiro passo): começamos observando que existe uma aplicação bimodal 
$g_{1}:[0,1] \rightarrow[0,1]$ com pontos extremos $c, d \in(0,1)$ tal que $g_{1}$ é crescente em $[0, c]$ e o primeiro retorno $\phi_{1}$ é do tipo $F_{5}$.

Indução (hipótese de indução): assumimos que existe uma aplicação bimodal $g_{3 k+1}$ : $[0,1] \rightarrow[0,1]$ cujos pontos extremos são $c, d \in(0,1)$ (os mesmos de $g_{1}$ ) e tal que $g_{3 k+1}$ é crescente em $[0, c]$ e para $1 \leq i \leq k$ os primeiros retornos $\phi_{3 i-1}$, $\phi_{3 i}$ e $\phi_{3 i+1}$ são dos tipos $F_{3}, \quad F_{4}, \quad F_{5}$, respectivamente (veja a Figura 4.1 ).

Indução (passo indutivo): este passo da indução consiste de 3 partes nas quais construiremos $g_{3 k+2}, g_{3 k+3}$ e $g_{3 k+4}$. Para construirmos $g_{3 k+2}$ consideramos $I_{3 k+1}, J_{3 k+1}$ os domínios críticos e $C_{3 k+1}, \quad D_{3 k+1}$ as domínios pós-críticos de $\phi_{3 k+1}$. Lembre-se que para todo $n \geq 0, \phi_{n}$ aplica $C_{n+1}$ sobre $I_{n}\left(\right.$ ou $\left.J_{n}\right)$ difeomorficamente. Definimos então $\tilde{C}_{n+1} \subset C_{n+1}$, o qual é a pré-imagem de $I_{n+1}\left(\right.$ ou $\left.J_{n+1}\right)$ por $\phi_{n}$. Da mesma forma definimos também $\tilde{D}_{n+1} \subset D_{n+1}$. Como $\phi_{3 k+1}$ é uma aplicacão de primeiro retorno podemos modificar $g_{3 k+1}$ em $I_{3 k+1} \cup J_{3 k+1}$ de modo que cada ramo não-crítico de $\phi_{3 k+1}$ nào se altere e os valores críticos $\phi_{3 k+1}(c)$ and $\phi_{3 k+1}(d)$ se movem independentemente para posições adequadas em $C_{3 k+1}$ e $D_{3 k+1}$, respectivamente. Então escolhemos $g_{3 k+2}$ de modo que $\phi_{3 k+1}$ é do tipo $F_{5}$ e $\phi_{3 k+2}(c) \in \phi_{3 k+1 \mid I_{3 k+1}}^{-1}\left(J_{3 k+1}\right)$ e $\phi_{3 k+2}(d) \in \phi_{3 k+1 \mid J_{3 k+1}}^{-1}\left(I_{3 k+1}\right)$. Em particular temos que a imagem dos ramos críticos de $\phi_{3 k+1}$ contêm os domínios destes ramos, mais precisamente $\phi_{3 k+1}\left(I_{3 k+1}\right) \supset J_{3 k+1}$ e $\phi_{3 k+1}\left(J_{3 k+1}\right) \supset I_{3 k+1}$. Definimos

$$
I_{3 k+2}:=\phi_{3 k+1 \mid I_{3 k+1}}^{-1}\left(\tilde{C}_{3 k+1}\right) \text { e } J_{3 k+2}:=\phi_{3 k+1 \mid J_{3 k+1}}^{-1}\left(\tilde{D}_{3 k+1}\right)
$$

Nestes domínios colocamos $\phi_{3 k+2}:=\phi_{3 k+1}^{2}$. Também definimos

$$
C_{3 k+2}:=\phi_{3 k+1 \mid I_{3 k+1}}^{-1}\left(J_{3 k+1}\right) \text { e } D_{3 k+2}:=\phi_{3 k+1 \mid J_{3 k+1}}^{-1}\left(I_{3 k+1}\right) .
$$

Nestes domínios colocamos $\phi_{3 k+2}:=\phi_{3 k+1}$. Fazendo mais uma modificação em $g_{3 k+2}$ de 
modo a ajustar as posições de $\phi_{3 k+2}(c)$ e $\phi_{3 k+2}(d)$ teremos que $\phi_{3 k+2}$ é do tipo $F_{3}$.

Agora escolhemos $g_{3 k+3}$ de modo que $\phi_{3 k+2}$ é do tipo $F_{3}$ e $\phi_{3 k+3}(c) \in \phi_{3 k+2 \mid J_{3 k+2}}^{-1}\left(J_{3 k+2}\right)$ e $\phi_{3 k+3}(d) \in \phi_{3 k+2 \mid I_{3 k+2}}^{-1}\left(I_{3 k+2}\right)$. Como antes temos que $\phi_{3 k+2}\left(I_{3 k+2}\right) \supset I_{3 k+2}$ e $\phi_{3 k+2}\left(J_{3 k+2}\right) \supset$ $J_{3 k+2}$. Então definimos

$$
I_{3 k+3}:=\phi_{3 k+2 \mid I_{3 k+2}}^{-1}\left(\dot{C}_{3 k+2}\right) \text { e } \quad J_{3 k+3}:=\phi_{3 k+2 \mid J_{3 k+2}}^{-1}\left(\tilde{D}_{3 k+2}\right) .
$$

Nestes domínios colocamos $\phi_{3 k+3}:=\phi_{3 k+2}^{2}$. Também definimos

$$
C_{3 k+3}:=\phi_{3 k+2 \mid I_{3 k+2}}^{-1}\left(I_{3 k+2}\right) \text { e } D_{3 k+3}:=\phi_{3 k+2 \mid J_{3 k+2}}^{-1}\left(J_{3 k+2}\right) .
$$

Nestes domínios colocamos $\phi_{3 k+3}:=\phi_{3 k+2}$. Modificando $g_{3 k+3}$ de modo a ajustar as posições de $\phi_{3 k+3}(c)$ e $\phi_{3 k+3}(d)$ teremos que $\phi_{3 k+3}$ é do tipo $F_{4}$.

Encerrando a indução escolhemos $g_{3 k+4}$ de modo que $\phi_{3 k+3}$ é do tipo $F_{4}$ e $\phi_{3 k+4}(c) \in$ $\phi_{3 k-+3 \mid J_{3 k+3}}^{-1}\left(I_{3 k+3}\right)$ e $\phi_{3 k+4}(d) \in \phi_{3 k+3 \mid I_{3 k+3}}^{-1}\left(J_{3 k+3}\right)$. De modo análogo ao do passo anterior temos que $\phi_{3 k+3}\left(I_{3 k+3}\right) \supset J_{3 k+3}$ e $\phi_{3 k+3}\left(J_{3 k+3}\right) \supset I_{3 k+3}$. Definimos

$$
I_{3 k+4}:=\phi_{3 k+3 \mid I_{3 k+3}}^{-1}\left(\tilde{C}_{3 k+3}\right) \text { e } J_{3 k+4}:=\phi_{3 k+3 \mid J_{3 k+3}}^{-1}\left(\tilde{D}_{3 k+3}\right) .
$$

Nestes domínios colocamos $\phi_{3 k+4}:=\phi_{3 k+3}^{2}$. Também definimos

$$
C_{3 k+4}:=\phi_{3 k+3 \mid J_{3 k+3}}^{-1}\left(I_{3 k+3}\right) \text { e } \quad D_{3 k+4}:=\phi_{3 k+3 \mid I_{3 k+3}}^{-1}\left(J_{3 k+3}\right) \text {. }
$$

Nestes domínios colocamos $\phi_{3 k+4}:=\phi_{3 k+3}$. Modificando $g_{3 k+4}$ de modo a ajustar as posições de $\phi_{3 k+4}(c)$ e $\phi_{3 k+4}(d)$ teremos que $\phi_{3 k+4}$ é do tipo $F_{5}$.

Podemos construir a sequência $g_{n}$ de modo que 
$\bigcap_{n=0}^{\infty} I_{n}=\{c\}$ e $\bigcap_{n=0}^{\infty} J_{n}=\{d\}$. Então $g_{n}$ converge, com $n$, para uma aplicação bimodal Fibonacci $g$. Como a família $P_{a b}$ é uma família completa (veja [MS], páginas 117-119) nós concluimmos que existem os parâmetros $a, b$ tais que $P_{a b}$ satisfaz o Teorema 2.3.1.

Finalmente gostariamos de enfatizar que nas definições dos tipos $F_{3}, F_{4}$ e $F_{5}$ nada exigimos sobre as orientações dos ramos da aplicação. Estas orientações não são relevantes para a construção de polinômios cúbicos Fibonacci ou eventualmente Fibonacci. No entanto se refinarmos a definição dos tipos de modo a levar em conta estas orientações poderemos dar mais detalhes sobre o comportamento da sequência $\phi_{n}$ associada a um polinômio cúbico Fibonacci.

Consideramos então os subtipos $F_{i}^{j k}$, onde $i=3,4,5$ e $j, k \in\{+,-\}$, sendo que $j=+$ e $j=-$ significam, respectivamente, que os ramos monótonos preservam ou invertem orientação enquanto que $k=+\mathrm{e} k=-$ significam, respectivamente, que o ramo crítico em $c$ tem concavidade para cima ou para baixo.

Com este refinamento teremos que tanto na família $P_{a b}$ como na $Q_{a b}$ os primeiros retornos $\phi_{n}$ exibem a seguinte sequência de tipos

$$
F_{5}^{+-} F_{3}^{--} F_{4}^{-+} F_{5}^{--} F_{3}^{++} F_{4}^{++} F_{5}^{++} F_{3}^{-+} F_{4}^{--} F_{5}^{-+} F_{3}^{+-} F_{4}^{+-} F_{5}^{+-} .
$$



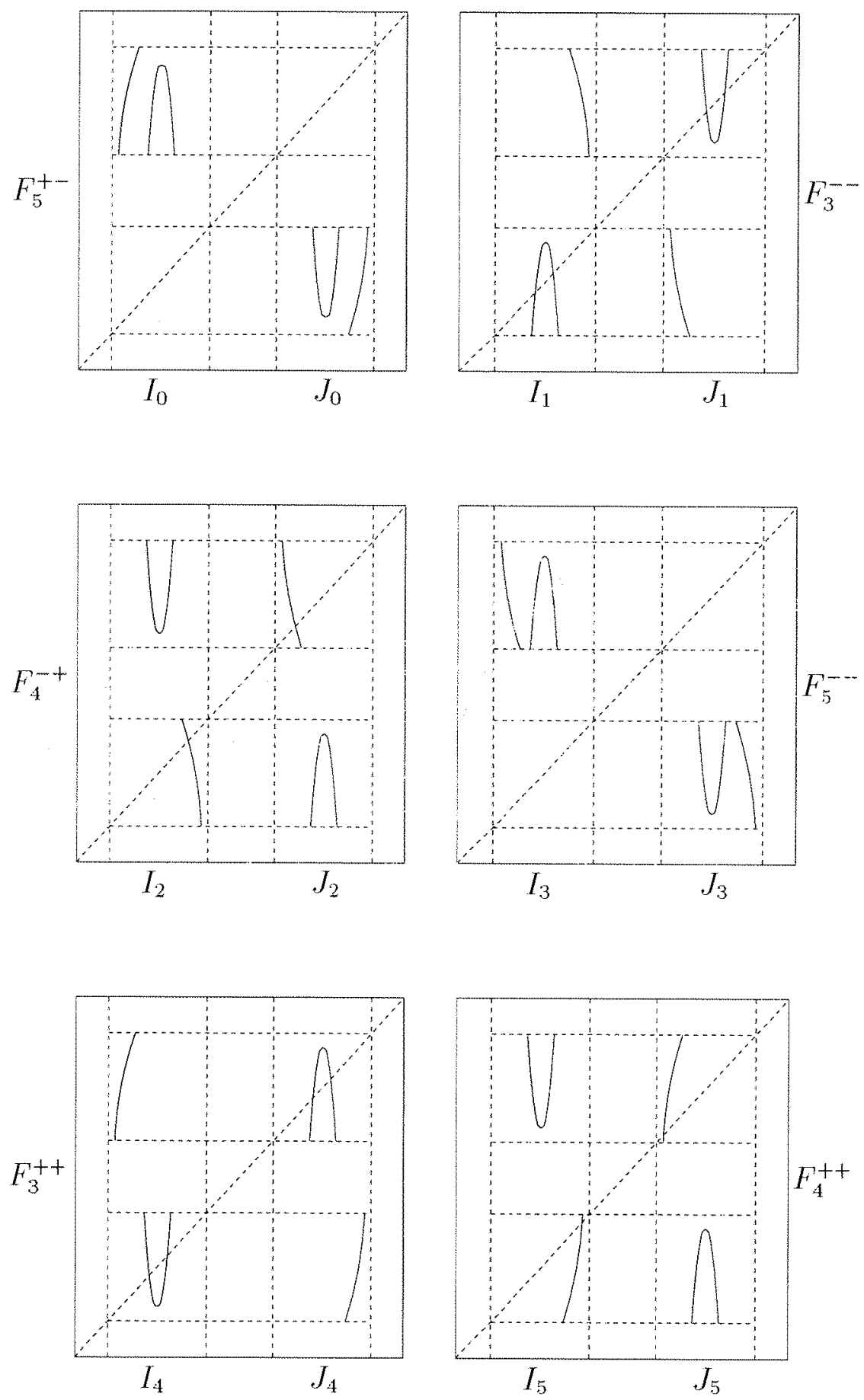

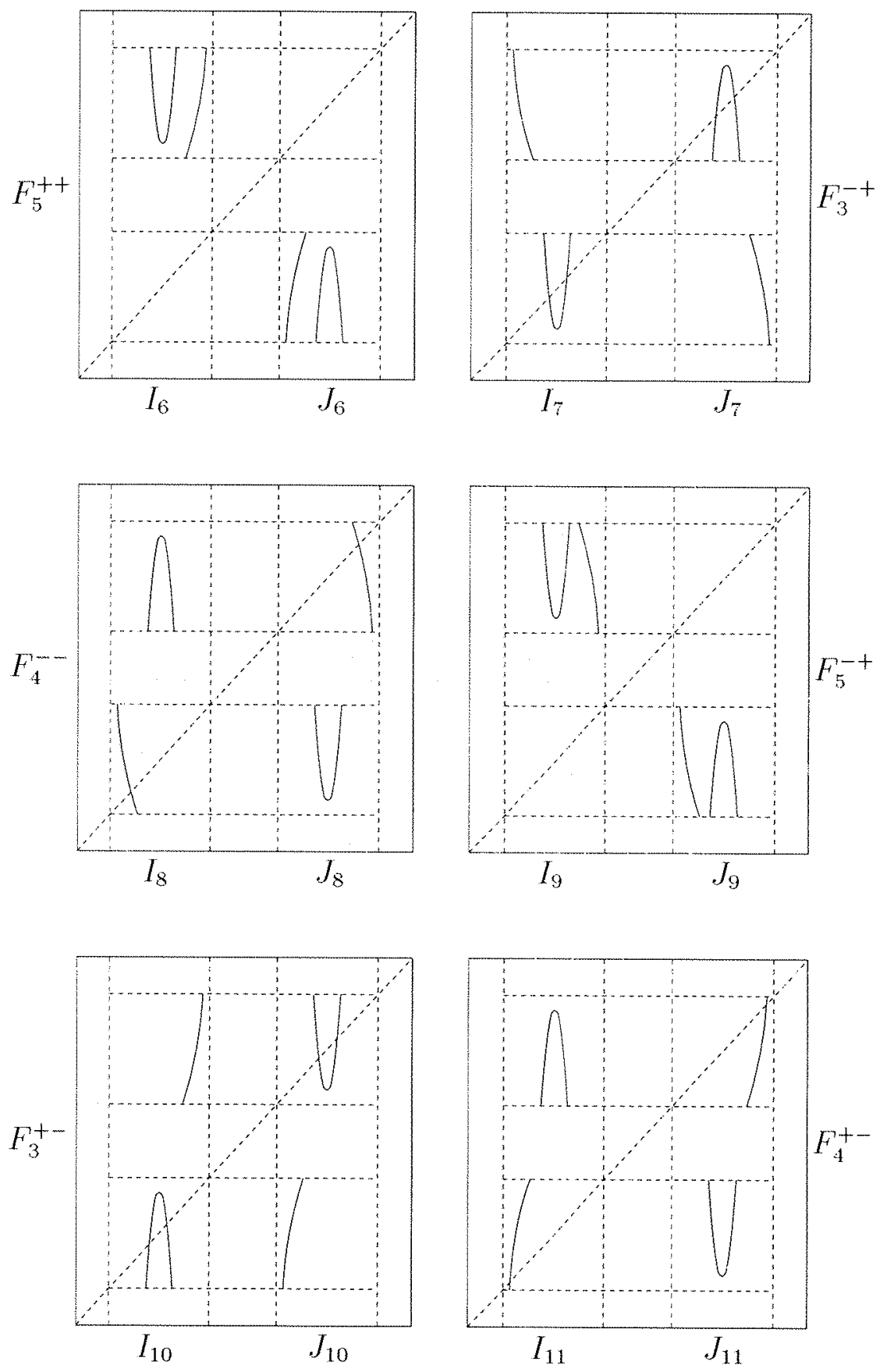

Figura 4.1: Seqüência de tipos de Fibonacci 


\subsection{Polinômios cúbicos eventualmente Fibonacci}

Dada $\phi_{n}$ a aplicação de primeiro retorno definida em $I_{n} \cup J_{n}$, denotamos por $C_{n}, D_{n}$ os domínios pós-críticos que contêm os valores críticos $\phi_{n}(c), \phi_{n}(d)$ respectivamente. Relembramos a definição de polinômios cúbicos eventualmente Fibonacci.

Definição 4.2.1 Se $f$ não possui retornos centrais e existe um inteiro positivo $n_{0}$ tal que para todo $n \geq n_{0} \phi_{n \mid C_{n}}=\phi_{n-1 \mid C_{n}}, \phi_{n \mid D_{n}}=\phi_{n-1 \mid D_{n}}, \phi_{n+1}(c)=\phi_{n \mid C_{n}} \circ \phi_{n \mid I_{n}}(c) \epsilon$ $\phi_{n+1}(d)=\phi_{n \mid D_{n}} \circ \phi_{n \mid J_{n}}(d)$ dizemos que $f$ é Eventualmente Fibonacci.

Provaremos a seguir que se $f$ é eventualmente Fibonacci, entào para $n=n_{0}$ a seqüência de aplicações de primeiro retorno $\phi_{n}, \phi_{n+1}, \ldots$ apresenta a sequência de tipos $F_{1} F_{2} F_{1} F_{2} \ldots$ ou $F_{3} F_{4} F_{5} F_{3} F_{4} F_{5} \ldots$ Este é o conteúdo do teorema 2.3 .2

\section{Prova do Teorema 2.3.2}

Primeiramente, vamos supor que $C_{n}, D_{n}$ estejam contidos em $I_{n-1}$. Como estes intervalos são restrições de $I_{n-1}$ pela aplicação $\phi_{n-1 \mid I_{n-1}}$ ambos intervalos são aplicados ou sobre $I_{n-1}$ ou sobre $J_{n-1}$. No primeiro caso acontece que $C_{n+1} \cup D_{n+1} \subset I_{n}$, e como $C_{n} \subset I_{n-1}$, temos que $\phi_{n \mid I_{n}}$ aplica $C_{n+1}, D_{n+1}$ sobre $I_{n}$. Assim temos novamente que $C_{n+2} \cup D_{n+2} \subset I_{n+1}$. Isto implica que os $\omega$-limites $\omega(c), \omega(d)$ não contêm o ponto crítico d. Este não é nosso caso. No segundo caso acontece o Tipo $F_{1}$. Quando os intervalos $C_{n}, D_{n}$ estiverem contidos em $J_{n-1}$, a situação será análoga.

Vamos supor agora que $C_{n}, D_{n}$ estejam contidos em intervalos diferentes. Temos então dois casos possíveis:

(Caso 1) $C_{n} \subset I_{n-1}, D_{n} \subset J_{n-1}$. Neste caso, existem quatro possibilidades: 
1. $\phi_{n-1}\left(C_{n}\right)=I_{n-1}, \phi_{n-1}\left(D_{n}\right)=J_{n-1}$. Neste caso acontece que para todo $n, C_{n} \subset$ $I_{n-1}$ e $D_{n} \subset J_{n-1}$, causando assim que $\omega(c)$ e $\omega(d)$ sejam diferentes. Este não é nosso caso.

2. $\phi_{n-1}\left(C_{n}\right)=J_{n-1}, \phi_{n-1}\left(D_{n}\right)=I_{n-1}$. Este é o Tipo $F_{3}$.

3. $\phi_{n-1}\left(C_{n}\right)=\phi_{n-1}\left(D_{n}\right)=I_{n-1}$. Neste caso, conforme o segundo item da definição de Fibonacci $C_{n+1}$ e $D_{n+1}$ estão em $I_{n}$. Como $C_{n}$ está em $I_{n-1}$, a aplicação $\phi_{n}$ definida em $I_{n}$ vai aplicar $C_{n+1}, D_{n+1}$ sobre $I_{n}$. Este não é nosso caso como vimos acima.

4. $\phi_{n-1}\left(C_{n}\right)=\phi_{n-1}\left(D_{n}\right)=J_{n-1}$. Neste caso, $C_{n+1}$ e $D_{n+1}$ estão em $J_{n}$. Como $D_{n}$ está em $J_{n-1}$, a aplicação $\phi_{n \mid J_{n}}$ vai aplicar $C_{n+1}, D_{n+1}$ sobre $J_{n}$. Este também não é nosso caso.

(Caso 2) $C_{n} \subset J_{n-1}, D_{n} \subset I_{n-1}$. Neste caso também existem quatro possibilidades:

1. $\phi_{n-1}\left(C_{n}\right)=\phi_{n-1}\left(D_{n}\right)=I_{n-1}$. Neste caso, $C_{n+1}, D_{n+1}$ estão em $I_{n}$ e, como $C_{n}$ está em $J_{n-1}$, a aplicação $\phi_{n \mid I_{n}}$ vai aplicar $C_{n+1}, D_{n+1}$ sobre $J_{n}$. Esté ó o Tipo $F_{1}$.

2. $\phi_{n-1}\left(C_{n}\right)=\phi_{n-1}\left(D_{n}\right)=J_{n-1}$. Este é análogo ao item anterior.

3. $\phi_{n-1}\left(C_{n}\right)=I_{n-1}, \phi_{n-1}\left(D_{n}\right)=J_{n-1}$. Este é o tipo $F_{5}$. Neste caso temos que $C_{n+1} \subset I_{n}$ e $C_{n+1}$ é aplicado sobre $J_{n}$ pois $\phi_{n}$ aplica $I_{n}$ em $J_{n-1}$. Temos também que $D_{n+1} \subset J_{n}$ e $D_{n+1}$ é aplicado sobre $I_{n}$, pois $\phi_{n}$ aplica $J_{n}$ em $I_{n-1}$. Este será então o Tipo $F_{3}$.

4. $\phi_{n-1}\left(C_{n}\right)=J_{n-1}, \phi_{n-1}\left(D_{n}\right)=I_{n-1}$. Neste caso, temos que $C_{n+1} \subset J_{n}, D_{n+1} \subset I_{n}$. Como $\phi_{n}: I_{n} \rightarrow J_{n-1}, \phi_{n}: J_{n} \rightarrow I_{n-1}$, resulta que $C_{n+1}$ é aplicado sobre $I_{n}$ e $D_{n+1}$ 
é aplicado sobre $J_{n}$. Este é o tipo $F_{5}$.

Mostraremos agora que os tipos $F_{1}, F_{2}$ e os tipos $F_{3}, F_{4}, F_{5}$ acontecem alternadamente. Vamos supor que $\phi_{n-1}$ é do tipo $F_{1}$. Neste caso, sabemos que $C_{n+1} \cup D_{n+1} \subset J_{n}$. Como $D_{n} \subset I_{n-1}$, temos que $\phi_{n}$ definida em $J_{n}$ aplica $C_{n+1}$ e $D_{n+1}$ sobre $I_{n}$, mostrando assim o tipo $F_{2}$. Novamente $C_{n+2} \cup D_{n+2} \subset I_{n+1}$ e como $C_{n+1} \subset J_{n}$, $\phi_{n+1}$ aplica $C_{n+2}$ e $D_{n+2}$ sobre $J_{n+1}$, voltando assim a ser o tipo $F_{1}$.

Vamos supor agora que $\phi_{n-1}$ é do tipo $F_{3}$. Como $\phi_{n-1}\left(C_{n}\right)=J_{n-1}$ e $\phi_{n-1}\left(D_{n}\right)=I_{n-1}$, temos que $C_{n+1} \subset J_{n}$ e $D_{n+1} \subset J_{n}$. Também pela posição de $C_{n}$ e $D_{n}$ sabemos que $\phi_{n}: I_{n} \rightarrow I_{n-1}$ e $\phi_{n}: J_{n} \rightarrow J_{n-1}$. Isto implica que $\phi_{n}: C_{n+1} \rightarrow J_{n}$ e $\phi_{n}: D_{n+1} \rightarrow I_{n}$. Este é o tipo $F_{4}$. Novamente, $C_{n+2} \subset J_{n+1}$ e $D_{n+2} \subset I_{n+1}$. Como $\phi_{n+1}: I_{n+1} \rightarrow J_{n}$ e $\phi_{n+1}: J_{n+1} \rightarrow I_{n}$, sabemos que $C_{n+2}$ e $D_{n+2}$ sào aplicados pela $\phi_{n+1}$ sobre $I_{n+1} \mathrm{e}$ $J_{n+1}$ respectivamente, resultando assim o tipo $F_{5}$. Nesta vez temos que $C_{n+3} \subset I_{n+2}$ e $D_{n+3} \subset J_{n+2}$. Como $\phi_{n+2}: I_{n+2} \rightarrow J_{n+1}$ e $\phi_{n+2}: J_{n+2} \rightarrow I_{n+1}$, temos que $C_{n+3}$ e $D_{n+3}$ sào aplicados pela $\phi_{n+3}$ sobre $J_{n+2}$ e $I_{n+2}$ respectivamente, resultando assim o tipo $F_{3}$. Isso conclui a prova.

\subsubsection{Tempos de retorno dos pontos críticos}

Nesta seção provaremos que os polinômios cúbicos Fibonacci têm os tempos mais rápidos possíveis de retorno apresentando assim a recorrência mais forte dos pontos críticos entre os polinômios cúbicos que não apresentam retornos centrais.

Note que para todo $n \geq 1$, existem números naturais $s_{n}=\tau_{n}(c), r_{n}=\tau_{n}(d)$ tal que $\phi_{n}(c)=f^{s_{n}}(c)$ e $\phi_{n}(d)=f^{r_{n}}(d)$. Assim podemos definir duas seqüências que são tempos de primeiros retornos dos seus pontos críticos $\tau(c)=\left(s_{1}, s_{2}, s_{3}, \ldots\right)$ e $\tau(d)=$ 
$\left(r_{1}, r_{2}, r_{3}, \ldots\right)$. Como temos visto no Teorema 2.3 .2 a combinatória de tipo Fibonacci pode começar a ocorrer a partir de um certo n-ésimo processo de indução na construção de aplicaşões de primeiro retorno. Por esta razão é provável que as seqüências $\tau(c), \tau(d)$ não coincidem (mesmo parcialmente a partir da ocorrência de tipo Fibonacci) com a seqüencia de Fibonacci $\alpha_{n}$, que é $\alpha_{1}=2, \alpha_{2}=3$ e $\alpha_{n+1}=\alpha_{n-1}+\alpha_{n}$ para todo $n \geq 2$. Entretanto, sob algumas hipóteses, mostraremos que as seqüências $\tau(c)$ e $\tau(d)$ satisfazem a propriedade da seqüência de Fibonacci, isto é: $s_{n+1}=s_{n-1}+s_{n}$ e $r_{n+1}=r_{n-1}+r_{n}$.

\section{Prova do Teorema 2.3 .3}

Vamos assumir que a combinatória de tipo Fibonacci ocorre a partir da primeira. etapa da induçào. Consideramos primeiro os polinômios cúbicos Fibonacci do tipo $F_{1} F_{2}$. Como $C_{1} \cup D_{1} \subset I_{0}$, obtemos que $s_{2}=s_{0}+s_{1}$ e $r_{2}=s_{0}+r_{1}$. E como $\phi_{1}$ aplica $C_{1} \cup D_{1}$ sobre $J_{0}$ os domínios $C_{2}$ e $D_{2}$ estão em $J_{1}$ e isto aplica que $s_{3}=r_{1}+s_{2}$ e $r_{3}=r_{1}+r_{2}$. Uma vez que $D_{1}$ está em $I_{0}$, sabemos que $\phi_{1}$ aplica. $J_{1}$ sobre $I_{0}$ e $C_{2} \cup D_{2}$ sobre $I_{1}$. Isto implica que $C_{3} \cup D_{3} \subset I_{2}$ e temos que $s_{4}=s_{2}+s_{3}$ e $r_{4}=s_{2}+r_{3}$. Sabemos que $C_{2} \subset J_{1}$. Isto implica que $\phi_{2}$ aplica $I_{2} \mathrm{em} J_{1}$ e, por isso, $C_{3} \cup D_{3}$ sobre $J_{2}$. Esta situação é igual à situação de $\phi_{1}$. Então, temos em geral

$$
\begin{array}{ll}
s_{2 n}=s_{2 n-2}+s_{2 n-1} & s_{2 n+1}=r_{2 n-1}+s_{2 n} \\
r_{2 n}=s_{2 n-2}+r_{2 n-1} & r_{2 n+1}=r_{2 n-1}+r_{2 n}
\end{array}
$$

Como $r_{2 n+1}-s_{2 n+1}=r_{2 n}-s_{2 n}$ e $r_{2 n}-s_{2 n}=r_{2 n-1}-s_{2 n-1}$, temos por induçâo que

$$
\begin{aligned}
s_{2 n+1} & =s_{2 n-1}+s_{2 n}+\left(r_{1}-s_{1}\right) \\
r_{2 n} & =r_{2 n-2}+r_{2 n-1}+\left(s_{1}-r_{1}\right)
\end{aligned}
$$

Disto temos que

$$
s_{2 n}=s_{2 n-2}+s_{2 n-1}
$$




$$
\begin{aligned}
& r_{2 n}=r_{2 n-2}+r_{2 n-1}+\left(s_{1}-r_{1}\right) \\
& s_{2 n+1}=s_{2 n-1}+s_{2 n}+\left(r_{1}-s_{1}\right) \\
& r_{2 n+1}=r_{2 n-1}+r_{2 n}
\end{aligned}
$$

Assumindo então que $s_{1}=r_{1}$, temos que $s_{n}=r_{n}$ para todo $n \geq 1$ e ainda as seqüências satisfazem a propriedade de Fibonacci.

A seguir, consideramos os polinômios cúbicos Fibonacci tipo $F_{3} F_{4} F_{5}$. Inicialmente assumiremos que $\phi_{1}$ é do tipo $F_{3}$. Então temos que $C_{1} \subset I_{0}$ e $D_{1} \subset J_{0}$ e isto implica que $s_{2}=s_{0}+s_{1}$ e $r_{2}=r_{0}+r_{1}$. Como $\phi_{1}$ aplica $C_{1}$ sobre $J_{0}$ e $D_{1}$ sobre $I_{0}$, temos que $C_{2} \subset J_{1}$ e $D_{2} \subset I_{1}$ e isto implica que $s_{3}=r_{1}+r_{2}$ e $r_{3}=s_{1}+r_{2}$. Como $C_{1} \subset I_{0}, D_{1} \subset J_{0}$, sabemos que $\phi_{1}$ aplica $I_{1}$ em $I_{0}$ e $J_{1}$ em $J_{0}$ e, por isso, $C_{2}$ sobre $J_{1}$ e $D_{2}$ sobre $I_{1}$. Isto implica que $C_{3} \subset J_{2}$ e $D_{3} \subset I_{2}$ e temos que $s_{4}=r_{2}+s_{3}$ e $r_{4}=s_{2}+r_{3}$. Aplicando o mesmo argumento, podemos verificar com facilidade que a situação dos domínios $C_{4} \mathrm{e}$ $D_{4}$ é igual à dos $C_{1}$ e $D_{1}$. Temos entào em geral

$$
\begin{array}{lll}
s_{3 n-1}=s_{3 n-3}+s_{3 n-2} & s_{3 n}=r_{3 n-2}+s_{3 n-1} & s_{3 n+1}=r_{3 n-1}+s_{3 n} \\
r_{3 n-1}=r_{3 n-3}+r_{3 n-2} & r_{3 n}=s_{3 n-2}+r_{3 n-1} & r_{3 n+1}=s_{3 n-1}+r_{3 n}
\end{array}
$$

A partir deste fato, observamos que

$$
\begin{aligned}
s_{3 n}-r_{3 n} & =\left(r_{3 n-2}-s_{3 n-2}\right)+\left(s_{3 n-1}-r_{3 n-1}\right) \\
& =\left(r_{3 n-2}-s_{3 n-2}\right)+\left(s_{3 n-3}-r_{3 n-3}\right)+\left(s_{3 n-2}-r_{3 n-2}\right) \\
& =s_{3(n-1)}-r_{3(n-1)} .
\end{aligned}
$$

E por indução temos que $s_{3 n}-r_{3 n}=s_{0}-r_{0}$. Temos também que

$$
s_{3 n+1}-r_{3 n+1}=\left(r_{3 n-1}-s_{3 n-1}\right)+\left(s_{3 n}-r_{3 n}\right)
$$




$$
\begin{aligned}
& =\left(r_{3 n-1}-s_{3 n-1}\right)+\left(r_{3 n-2}-s_{3 n-2}\right)+\left(s_{3 n-1}-r_{3 n-1}\right) \\
& =s_{3(n-1)+1}-r_{3(n-1)+1} .
\end{aligned}
$$

E por indução temos que $s_{3 n+1}-r_{3 n+1}=(-1)^{n}\left(s_{1}-r_{1}\right)$. Usando esses últimos dois resultados e o anterior, temos que

$$
\begin{gathered}
s_{3 n-1}=s_{3 n-3}+s_{3 n-2} \\
r_{3 n-1}=r_{3 n-3}+r_{3 n-2} \\
s_{3 n}=s_{3 n-2}+s_{3 n-1}+(-1)^{n}\left(r_{1}-s_{1}\right) \\
r_{3 n}=r_{3 n-2}+r_{3 n-1}+(-1)^{n}\left(s_{1}-r_{1}\right) \\
s_{3 n+1}=s_{3 n-1}+s_{3 n}+(-1)^{n}\left(r_{1}-s_{1}\right)+\left(r_{0}-s_{0}\right) \\
r_{3 n+1}=r_{3 n-1}+r_{3 n}+(-1)^{n}\left(s_{1}-r_{1}\right)+\left(s_{0}-r_{0}\right)
\end{gathered}
$$

Assumindo $s_{0}=r_{0}$ e $s_{1}=r_{1}$ temos que $s_{n}=r_{n}$ para todo $n \geq 1$ e as seqüencias $\tau(c)$ e $\tau(d)$ satisfazem a propriedade de Fibonacci.

Assim mostramos que se a aplicação de primeiro retorno começa a apresentar tipo Fibonacci a partir da $n$-ésima etapa e nessa etapa se satisfazem $s_{n}=r_{n}$ e $s_{n-1}=r_{n-1}$, então a partir desse momento os tempos de primeiro retorno de cada tipo Fibonacci satisfaz a propriedade de Fibonacci. Em particular, se a combinatória de tipo Fibonacci ocorre desde o início da indução e satisfaz a condiçào inicial, ou seja, $s_{0}=r_{0}=1 \mathrm{e}$ $s_{1}=r_{1}=2$, então as seqüências $s_{n}$ e $r_{n}$ são iguais à seqüência de Fibonacci $\alpha_{n}$.

Agora provaremos que os polinômios cúbicos Fibonacci tem menor tempo de retorno entre os polinômios que não apresentam retornos centrais. 


\section{Prova do Teorema 2.3.4}

Note que, se $f$ não possui retornos centrais, $C_{n}$ e $D_{n}$ estão contidos em $\left(I_{n-1} \backslash I_{n}\right) U$ $\left(J_{n-1} \backslash J_{n}\right)$ para todo $n \geq 1$ e que os intervalos $I_{n+1}, J_{n+1}$ são definidos como sendo préimagens de $C_{n}, D_{n}$ por $\phi_{n}$ respectivamente. Temos assim que $\phi_{n+1 \mid I_{n+1}}=\phi_{n \mid C_{n}} \circ \phi_{n \mid I_{n}} \mathrm{e}$ $\phi_{n+1 \mid J_{n+1}}=\phi_{n \mid D_{n}} \circ \phi_{n \mid J_{n}}$. No caso de Fibonacci temos que $\phi_{n \mid C_{n} \cup D_{n}}=\phi_{n-1 \mid I_{n-1} \cup J_{n-1}}$. Isso implica que $s_{n+1}=s_{n-1}+s_{n}$. No caso de não Fibonacci $\phi_{n-1 \mid l_{n-1} \cup J_{n-1}}$ aplica $C_{n}$ em $I_{n-2} \backslash I_{n-1}$ ou em $J_{n-2} \backslash J_{n-1}$. Sendo assim, o tempo do primeiro retorno do valor critico $f^{s_{n}}(c) \in C_{n}$ ao intervalo $I_{n}$ fica maior que $s_{n-1}$. Isto é $s_{n+1}>s_{n}+s_{n-1}$. Isto mostra que, excluídos retornos-centrais, a aplicação do tipo Fibonacci tem o menor tempo de primeiro retorno, isto é, se uma aplicação $g$ tem tempo de primeiro retorno $s_{n+1}(g)<\alpha_{n+1}$, verifica-se que $g$ possui retornos centrais.

\subsection{As seqüências de kneading dos polinômios cúbicos Fibonacci}

Na teoria qualitativa dos sistemas dinâmicos a relação de equivalência naturaî para expressar a noção de "mesma dinâmica" é o que chamamos conjugação, a saber: duas aplicações $f$ e $g$ são topologicamente conjugadas se existe um homeomorfismo $h: I \rightarrow I$ tal que $h \circ f=g \circ h$. Um tal homeomorfismo, chamado uma conjugação entre $f$ e $g$, aplica órbitas de $f$ em órbitas de $g$ e preserva propriedades topológicas do sistema. A relação de conjugação topológica entre duas aplicações está relacionada com a questão de mesma seqüência de kneading dos valores críticos. Nesta seção vamos construir as seqüências de kneading no caso de polinômios cúbicos Fibonacci. 
Dado $x \in I$ definimos o seu itinerário $I(x)=\left(i_{1}(x), i_{2}(x), \cdots\right)$, pondo

$$
i_{j}(x)=\left\{\begin{array}{cc}
1, & \text { se } f^{j}(x)<c \\
2, & \text { se } c<f^{j}(x)<d \\
3, & \text { se } f^{j}(x)>d
\end{array}\right.
$$

Definimos então as seqüências de kneading $K_{f}(c), K_{f}(d)$ como sendo os itinerários de $c$ e $d$, respectivamente. Lembramos que para todo $n \geq 1$ existem $s_{n}, r_{n}$ tais que $\phi_{n}(c)=f^{s_{n}}(c)$ e $\phi_{n}(d)=f^{r_{n}}(d)$. No caso Polinômio cúbico Fibonacci verifica-se que $s_{n}=r_{n}$.

Proposição 4.3.1 Dado um polinômio cúbico Fibonacci cujos pontos críticos são recorrentes e possuem o mesmo $\omega$-limite temos que as seqüências de kneading $K_{f}(c), K_{f}(d)$ de modo que $i_{1}(c)=i_{s_{1}}(c)=3, i_{1}(d)=i_{s_{1}}(d)=1, i_{s_{2}}(c)=i_{s_{2}}(d)=2$ e para todo $n \geq 2$

1. Se $C_{n} \subset I_{n-1}$

$$
\left(i_{s_{n}+1}(c), \ldots i_{s_{n+1}-1}(c), i_{s_{n+1}}(c)\right)=\left(i_{1}(c), \ldots, i_{s_{n-1}-1}(c), i_{s_{n+1}}(c)\right)
$$

2. Se $C_{n} \subset J_{n-1}$

$$
\left(i_{s_{n}+1}(c), \ldots i_{s_{n+1}-1}(c), i_{s_{n+1}}(c)\right)=\left(i_{1}(d), \ldots, i_{s_{n-1}-1}(d), i_{s_{n+1}}(c)\right)
$$

Prova: Primeiro passo: Pela definição de $f$ temos que $i_{1}(c)=i_{2}(c)=3 \mathrm{e}$ $i_{1}(d)=i_{2}(d)=1$. Observamos que $\phi_{2}(c)=f^{3}(c) \in C_{2}$ e $\phi_{2}(d)=f^{3}(c) \in D_{2}$. Segundo os subtipos mostrados anteriormente, temos que $C_{2} \subset(c, d), D_{2} \subset(c, d)$. Isso resulta que $i_{s_{2}}(c)=2, i_{s_{2}}(d)=2$. Como $C_{2} \subset I_{1}$ temos que os itinerários de $\phi_{2}(c)$ e $c$ coincidem ao longo da iterada pela $\phi_{1}$ até o nível $s_{1}-1$. O mesmo corre com $\phi_{2}(d)$ e $d$. Como 
$s_{1}=2$, obtemos que $i_{4}(c)=i_{s_{2}-1}(c)=i_{1}(c)$ e $i_{4}(d)=i_{s_{2}-1}(d)=i_{1}(d)$. Também como $C_{3} \subset(c, d)$ e $D_{3} \subset(c, d)$, para $s_{3}=5$, verifica-se que $i_{s_{3}}(c)=i_{s_{3}}(d)=2$.

Passo indutivo: Suponha que temos a sequência de kneading até o nível de $s_{n}$ para $c$ e $d$. Consideramos o primeiro caso, isto é: $C_{n} \subset I_{n-1}$. Nesta situação temos que os itinerários de $\phi_{n}(c)$ e $c$ coincidem ao longo da iterada pela $\phi_{n-1}$, ou seja, durante o tempo $s_{n-1}-1$. Assim temos que

$$
\left(i_{s_{n}+1}(c), \ldots i_{s_{n}+s_{n-1}-1}(c)\right)=\left(i_{1}(c), \ldots, i_{s_{n-1}-1}(c)\right) .
$$

Lembramos que a aplicação $f$ é Fibonacci, então temos que $s_{n}+s_{n-1}-1=s_{n+1}-1$. Então basta definirmos $i_{s_{n+1}}(c)$ dependendo de onde $f^{s_{n+1}}(c)$ está contido. Caso $C_{n} \subset J_{n-1}$, temos que os itinerários de $\phi_{n}(c)$ e $d$ coincidem ao longo da iterada pela $\phi_{n-1}$ durante o tempo $s_{n-1}-1$. Assim temos a seqüência de kneading até o nível $s_{n+1}$. A construçào de kneading para d será análoga.

Por fim apresentamos as seqüências de kneading dos polinômios cúbicos Fibonacci:

$$
\begin{aligned}
& K_{f}(c)=(3, \quad 3, \quad 2,3,2, \quad 1,1,3,1,1,2,1,1,3,3,2,3,2,1,1,2, \ldots) \\
& K_{f}(d)=(1, \quad 1, \quad 2, \quad 1,2, \quad 3,3,1, \quad 3,3,2,3,3,1,1,2,1,2,3,3,2, \ldots)
\end{aligned}
$$

Os espaços maiores entre os números da seqüência ocorrem exatamente nos números da seqüencia de Fibonacci, isto é: $1,2,3,5,8,13, \ldots$ 


\section{Capítulo 5}

\section{Decaimento de Geometria}

\subsection{Crescimento dos anéis principais}

A estrátegia que utilizamos para provar o decaimento de geometria enunciado no Teorema 2.3.5 é a de olhar para as continuaçóes analíticas $\Phi_{n}$ das aplicaçôes de primeiro retorno $\phi_{n}$. Dadas então as sequências de discos topológicos $\mathcal{I}_{0} \supset \mathcal{I}_{1} \supset \mathcal{I}_{2} \supset \ldots \supset\{c\} \mathrm{e}$ $\mathcal{J}_{0} \supset \mathcal{J}_{1} \supset \mathcal{J}_{2} \supset \ldots\{d\}$ construídas na Seção 3.2 .2 analisamos as sequências de módulos dos anéis $\mathcal{I}_{n} \backslash \overline{\mathcal{I}_{n+1}}$ e $\mathcal{J}_{n} \backslash \overline{\mathcal{J}_{n+1}}$ que chamamos de anéis principais. O Teorema 5.1 .1 a seguir garante o crescimento linear destes módulos e o decaimento exponencial de geometria é uma consequência deste fato, veja [LV].

Teorema 5.1.1 Seja $f=P_{a b}$ ou $f=Q_{a b}$ um polinômio cúbico Fibonacci cujos pontos críticos são recorrentes e possuem o mesmo w-limite. Então existe uma costante $C>0$ tal que para todo $n>0$,

$$
\bmod \left(\mathcal{I}_{n} \backslash \overline{\mathcal{I}_{n+1}}\right) \geq C \cdot n \quad e \bmod \left(\mathcal{J}_{n} \backslash \overline{\mathcal{J}_{n+1}}\right) \geq C \cdot n
$$


Uma vez provado o Teorema 5.1.1, temos o Corolário 5.1.1 abaixo que garante o decaimento exponencial de geometria do Teorema 2.3.5 como queremos.

Corolário 5.1.1 Seja $f=P_{a b}$ ou $f=Q_{a b}$ um polinômio cúbico eventualmente Fibonacci cujos pontos críticos são recorrentes e possuem o mesmo w-limite. Entäo existe uma constante $C_{1}>0$ tal que para todo $n>0$,

$$
\log \frac{\left|I_{n}\right|}{\left|I_{n+1}\right|} \geq C_{1} \cdot n \quad \text { e } \log \frac{\left|J_{n}\right|}{\left|J_{n+1}\right|} \geq C_{1} \cdot n
$$

Prova: Veja a prova deste Corolário em [LV].

\subsection{Anéis principais no caso eventualmente Fibo- nacci}

Seja $\phi_{n}$ a sequência de primeiros retornos a $I_{n-1} \cup J_{n-1}$ associados a um polinômio cúbico eventualmente Fibonacci $f=P_{a b}$ ou $f=Q_{a b}$. Estamos interessados apenas nas sequèncias de ramos críticos $\phi_{n \mid I_{n}}, \phi_{n \mid J_{n}}$ e pós-críticos $\phi_{n \mid C_{n}}, \phi_{n \mid D_{n}}$. Consideramos as suas respectivas continuações analíticas $\Phi_{n \mid \mathcal{I}_{n}}, \quad \Phi_{n \mid \mathcal{J}_{n}}, \quad \Phi_{n \mid \mathcal{C}_{n}}$ e $\Phi_{n \mid \mathcal{D}_{n}}$ como definidas na Seção 3.2.2.

Como $f$ é eventualmente Fibonacci pelo Teorema 2.3.2 podemos assumir que para um dado $n_{0}$ suficientemente grande $\phi_{n_{0}}$ é do tipo $F_{1} F_{2}$ ou $F_{3} F_{4} F_{5}$. Teremos então dois casos para analisar. 


\subsubsection{Polinômios eventualmente Fibonacci do tipo $F_{1} F_{2}$}

Assumimos que $\phi_{n_{0}}$ é do tipo $F_{1}$ e pelo Teorema 2.3 .2 temos que $\phi_{n_{0}+2 i}$ e $\phi_{n_{0}+2 i+1}$, para $i \geq 0$, são do tipo $F_{1}$ e $F_{2}$, respectivamente. Dado então $\phi_{n}$ do tipo $F_{1}$ consideramos a sua continuação analítica $\Phi_{n}$ e vamos relembrar a sua dinâmica. Temos que $\mathcal{C}_{n}, \mathcal{D}_{n} \subset \mathcal{I}_{n-1}$ e são aplicados sobre $\mathcal{J}_{n-1}$. Isto também já determina que $\mathcal{I}_{n}$ e $\mathcal{J}_{n}$ são aplicados sobre $\mathcal{I}_{n-1}$. No entanto, para estimar os módulos dos anéis principais vamos considerar os domínios $\tilde{\mathcal{C}}_{n} \subset \mathcal{C}_{n}$ e $\tilde{\mathcal{D}}_{n} \subset \mathcal{D}_{n}$, os quais são aplicados sobre $\mathcal{J}_{n}$.

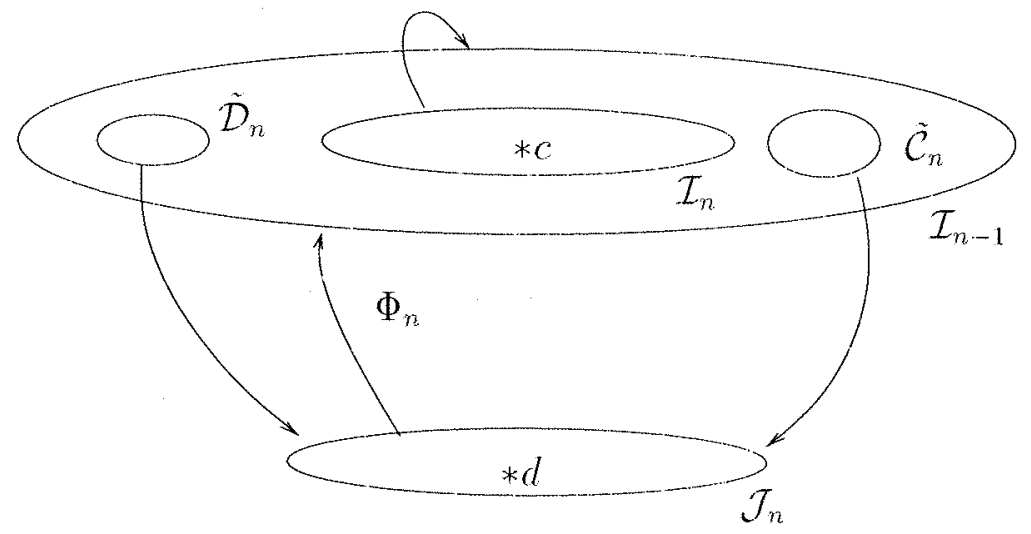

Figura 5.1: Fibonacci Tipo $F_{1}$

Agora definimos os domínios $\mathcal{I}_{n+1}, \mathcal{J}_{n+1}$ pela pré-imagem dos domínios $\tilde{\mathcal{C}}_{n}, \tilde{\mathcal{D}}_{n}$ respectivamente e também definimos $\mathcal{C}_{n+1}, \mathcal{D}_{n+1} \subset \mathcal{J}_{n}$ como pré-imagens de $\mathcal{I}_{n}$. Temos assim que $\Phi_{n+1}$ é do tipo $F_{2}$, ou seja, $\mathcal{C}_{n+1}, \mathcal{D}_{n+1} \subset \mathcal{J}_{n}$ e são aplicados sobre $\mathcal{I}_{n}$. Temos $\Phi_{n+1}=\Phi_{n}$ nestes domínios. Fica determinado que $\mathcal{I}_{n+1}$ e $\mathcal{J}_{n+1}$ são aplicados sobre $\mathcal{J}_{n}$ e $\Phi_{n+1}=\Phi_{n}^{2}$ nestes domínios.

Em seguida definimos $\mathcal{I}_{n+2}, \mathcal{J}_{n+2}$ como pré-imagens de $\mathcal{C}_{n}, \mathcal{D}_{n}$ respectivamente e $\tilde{\mathcal{C}}_{n+2}, \tilde{\mathcal{D}}_{n+2}$ como pré-imagens de $\mathcal{J}_{n+2}$. Assim temos que $\Phi_{n+2}$ é do tipo $F_{1}$ novamente. 
Temos então que $\tilde{\mathcal{C}}_{n+2}, \tilde{\mathcal{D}}_{n+2} \subset \mathcal{I}_{n+1}$ e são aplicados sobre $\mathcal{J}_{n+2}$. Ocorre que $\Phi_{n+2}=\Phi_{n+1}$ nestes domínios. Também temos que $\mathcal{I}_{n+2}$ e $\mathcal{J}_{n+2}$ são aplicados sobre $\mathcal{I}_{n}$ e $\Phi_{n+2}=\Phi_{n+1}^{2}$ nestes domínios. Continuando a indução, observamos que para $k=0,1,2, \ldots$, se satisfaz que $\mathcal{I}_{n+2 k}$ é o domínio do primeiro-retorno a $\mathcal{I}_{n+2 k-2}$.

Provaremos o crescimento linear do módulo do anel $\left(\mathcal{I}_{n+2 k-2} \backslash \overline{\mathcal{I}_{n+2 k}}\right)$. Para isto escolhemos um sistema de 6 anéis $A_{n}^{1}, \ldots, A_{n}^{6}$ associados a $\tilde{\mathcal{C}}_{n}$ e a $\tilde{\mathcal{D}}_{n}$ dentro de $\mathcal{I}_{n-2}$. No caso em que $\Phi_{n}$ é do tipo $F_{1} F_{2}$ estes anéis satisfazem as seguintes propriedades:

- Todos os anéis estão contidos em $\mathcal{I}_{n-2}$.

- O disco topológico $\mathcal{I}_{n}$ está contido na componente limitada do complementar de $A_{n}^{2}$ e os discos topológicos $\tilde{\mathcal{C}}_{n}$ e $\tilde{\mathcal{D}}_{n}$ estão contidos na componente ilimitada.

- O anel $A_{n}^{1}$ está contido na interseçào de $\mathcal{I}_{n-2}$ com a componente ilimitada do complementar de $A_{n}^{2}$.

- O disco topológico $\mathcal{I}_{n}$ está contido na componente ilimitada do complementar de $A_{n}^{3} \cup A_{n}^{5}$.

- O anel $A_{n}^{4}$ está contido na interseção de $\mathcal{I}_{n-2}$ com a componente ilimitada do complementar de $A_{n}^{3}$, e o anel $A_{n}^{6}$ está contido na interseção de $\mathcal{I}_{n-2}$ com a componente ilimitada do complementar de $A_{n}^{5}$.

A partir destes 6 anéis construiremos indutivamente os 6 anéis $A_{n+1}^{1}, \ldots, A_{n+1}^{6}$ da etapa $n+1$. Isto será feito tomando pré-imagens por $\Phi_{n \mid \tau_{n}}$.

$$
A_{n+1}^{2}:=\Phi_{n \mid J_{n}}^{-1}\left(A_{n}^{5}\right)
$$




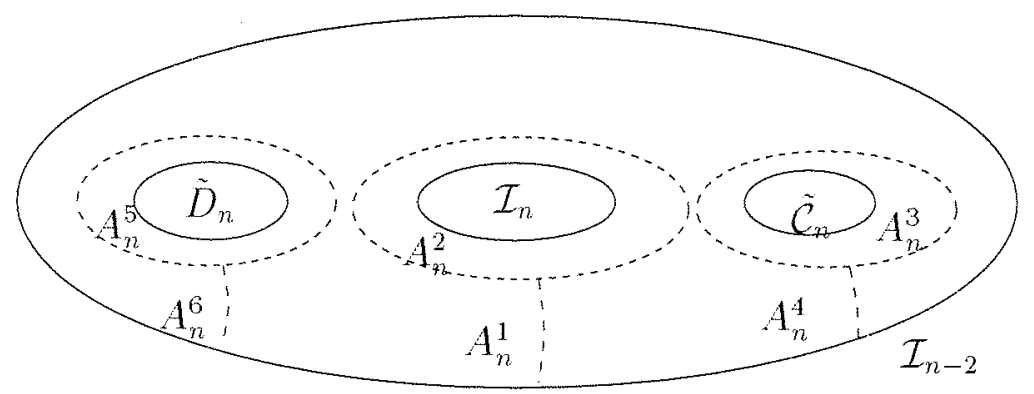

Figura 5.2: 6 anéis do Fibonacci Tipo $F_{1}$

$$
\begin{aligned}
& A_{n+1}^{1}:=\Phi_{n \mid \mathcal{J}_{n}}^{-1}\left(A_{n}^{6}\right) \\
& A_{n+1}^{3}:=\Phi_{n \mid \mathcal{J}_{n}}^{-1}\left(A_{n}^{2}\right) \\
& A_{n+1}^{5}:=\Phi_{n \mid \mathcal{J}_{n}}^{-1}\left(A_{n}^{2}\right) .
\end{aligned}
$$

Lembre-se que $A_{n}^{2}$ tem duas pré-imagens, então definimos $A_{n+1}^{3}$ como sendo uma pré-imagem de $A_{n}^{2}$ que contém $\tilde{\mathcal{C}}_{n+1}$ na componente limitada do seu complementar, e definimos $A_{n+1}^{5}$ como outra pré-imagem de $A_{n}^{2}$ que contém $\tilde{\mathcal{D}}_{n+1}$ na componente limitada do seu complementar. Definimos também $A_{n+1}^{4}$ como sendo a interseção de $\mathcal{J}_{n}$ com a componente ilimitada de complementar de $A_{n+1}^{3}$ e $A_{n+1}^{6}$ como sendo a interseção de $\mathcal{J}_{n}$ com a componente ilimitada de complementar de $A_{n+1}^{5}$. Os 6 anéis assim construídos satisfazem as propriedades referidas acima. Assim podemos continuar a indução para construir os 6 anéis $A_{n+2}^{1}, \ldots, A_{n+2}^{6}$ da etapa $n+2$ tomando pré-imagens por $\Phi_{n+1 \mid I_{n+1}}$.

Dados os 6 anéis de toda etapa da indução definiremos um sistema de 6 números reiais de modo que:

$$
\begin{aligned}
& a_{n}^{2} \leq \bmod A_{n}^{2}, \quad a_{n}^{1} \leq \bmod A_{n}^{1}+\bmod A_{n}^{2} \\
& a_{n}^{3} \leq \bmod A_{n}^{3}, \quad a_{n}^{4} \leq \bmod A_{n}^{3}+\bmod A_{n}^{4}
\end{aligned}
$$




$$
a_{n}^{5} \leq \bmod A_{n}^{5}, \quad a_{n}^{6} \leq \bmod A_{n}^{5}+\bmod A_{n}^{6}
$$

Lema 5.2.1 Para todo $n \geq n_{0}$, podemos escolher $a_{n}^{3}, \ldots a_{n}^{6}$ da seguinte maneira:

$$
a_{n}^{3}=a_{n}^{5}, \quad \text { e } \quad a_{n}^{4}=a_{n}^{6} .
$$

Prova: Da construção dos anéis temos que os anéis $A_{n+1}^{3}$ e $A_{n+1}^{5}$ são definidos como sendo pré-imagens do anel $A_{n}^{2}$ por uma aplicação univalente. Então temos que

$$
\bmod A_{n+1}^{3}=\bmod A_{n+1}^{5}=\bmod A_{n}^{2}
$$

Assim podemos escolher $a_{n+1}^{3}, a_{n+1}^{5}$ de tal modo que $a_{n+1}^{3}=a_{n+1}^{5}$.

Agora consideramos o módulo de $A_{n+1}^{4}$. Considere a aplicação de Riemann $h$ que aplica o anel $\{z: d<|z|<1\}$ sobre o anel $A_{n}^{1}$. Seja $t=\left|h^{-1}\left(\Phi_{n}(d)\right)\right|$. Então podemos dividir o anel $A_{n}^{1}$ em dois anéis, $V_{i}:=h(\{z: d<|z|<t\})$ e $V_{0}:=h(\{z: t<|z|<1\})$. Temos que $\bmod A_{n}^{1}=\bmod V_{i}+\bmod V_{o}$. Dividimos também o anel $A_{n}^{4}$ em dois anéis,

$$
U_{i}:=\Phi_{n \mid J_{n}}^{-1}\left(V_{i}\right) \text { e } U_{o}:=\Phi_{n_{i} \mid J_{n}}^{-1}\left(V_{o}\right)
$$

Note que a aplicação $\Phi_{n}$ aplica $U_{i}$ difeomorficamente sobre $V_{i}$ e aplica $U_{o}$ sobre $V_{0}$ como um recobrimento de grau 2. Como $A_{n+1}^{4}=U_{i} \oplus U_{o}$ temos que

$$
\bmod A_{n+1}^{4}=\bmod V_{i}+\frac{1}{2} \bmod V_{o}>\frac{1}{2} \bmod A_{n}^{1}
$$

O mesmo se verifica para o módulo do anel $A_{n+1}^{6}$. Portanto, podemos escolher $a_{n}^{4}, a_{n}^{6}$ de modo que $a_{n}^{4}=a_{n}^{6}$.

\section{Corolário 5.2.1}

$$
\bmod \left(\mathcal{I}_{n-1} \backslash \overline{\mathcal{I}_{n}}\right)=\bmod \left(\mathcal{J}_{n-1} \backslash \overline{\mathcal{J}_{n}}\right)
$$


Prova: Do Lema 5.2.1 temos que $\bmod A_{n-1}^{3}=\bmod A_{n-1}^{5}$ e $\bmod A_{n-1}^{4}=\bmod A_{n-1}^{6}$. De $[\mathrm{LV}]$ temos que $\bmod \left(A_{n-1}^{3} \oplus A_{n-1}^{4}\right)=\bmod \left(A_{n-1}^{5} \oplus A_{n-1}^{6}\right)$. Como $\mathcal{I}_{n-1} \backslash \overline{\mathcal{I}_{n}}$ é préimagem de $A_{n-1}^{3} \oplus A_{n-1}^{4}$ e $\mathcal{J}_{n-1} \backslash \overline{\mathcal{J}_{n}}$ é pré-imagem de $A_{n-1}^{5} \oplus A_{n-1}^{6}$ ambos por uma aplicação de recobrimento de grau 2 , isto implica que os anéis $\mathcal{I}_{n-1} \backslash \overline{\mathcal{I}_{n}}$ e $\mathcal{J}_{n-1} \backslash \overline{\mathcal{J}_{n}}$ têm o mesmo módulo.

O Lema 5.2.1 nos permite trabalhar apenas com o sistema de quatro números reais $a_{n}^{i}, i=1, \ldots, 4$ para estimar os módulos dos anéis. Provaremos a seguir que os módulos dos anéis principais não decrescem.

Proposição 5.2.1 Existe uma matriz A tal que para todo $n \geq n_{0} \operatorname{com} \Phi_{n}$ do tipo $F_{1}$, que satisfaz

$$
\left(a_{n+2}^{1}, a_{n+2}^{2}, a_{n+2}^{3}, a_{n+2}^{4}\right)=\left(a_{n}^{1}, a_{n}^{2}, a_{n}^{3}, a_{n}^{4}\right) \mathbf{A} .
$$

Além disso, a matriz A tem um auto valor igual a 1 e seu correspondente autovetor possui todas as componentes não negativas.

Prova: Vamos estimar os módulos dos anéis da etapa $n+1$ por

$$
\begin{aligned}
& A_{n+1}^{2}=\Phi_{n}^{-1}\left(A_{n}^{5}\right) \Rightarrow a_{n+1}^{2}=\frac{1}{2} a_{n}^{3} \\
& A_{n+1}^{1}=\Phi_{n}^{-1}\left(A_{n}^{6}\right) \Rightarrow a_{n+1}^{1}=\frac{1}{2} a_{n}^{4} \\
& A_{n+1}^{3}=\Phi_{n}^{-1}\left(A_{n}^{2}\right) \Rightarrow a_{n+1}^{3}=a_{n}^{2} \\
& A_{n+1}^{4}=\Phi_{n}^{-1}\left(A_{n}^{1}\right) \Rightarrow a_{n+1}^{4}=\frac{1}{2}\left(a_{n}^{1}+a_{n}^{2}\right)
\end{aligned}
$$

Vamos estimar também os módulos dos anéis da etapa $n+2$ por

$$
A_{n+2}^{2}=\Phi_{n+1 \mid I_{n+1}}^{-1}\left(A_{n+1}^{3}\right) \Rightarrow a_{n+2}^{2}=\frac{1}{2} a_{n+1}^{3}
$$




$$
\begin{aligned}
A_{n+2}^{1}=\Phi_{n+1 \mid \mathcal{I}_{n+1}}^{-1}\left(A_{n+1}^{4}\right) \oplus\left(\mathcal{I}_{n} \backslash \overline{\mathcal{I}_{n+1}}\right) & \Rightarrow a_{n+2}^{1}=\frac{1}{2} a_{n+1}^{4}+a_{n+1}^{1} \\
A_{n+2}^{3}=\Phi_{n+1 \mid \mathcal{I}_{n+1}}^{-1}\left(A_{n+1}^{2} \oplus\left(\mathcal{J}_{n+1} \backslash \overline{\mathcal{J}_{n+2}}\right)\right) & \Rightarrow a_{n+2}^{3}=a_{n+1}^{2}+\frac{1}{2} a_{n+1}^{4} \\
A_{n+2}^{4}=\Phi_{n+1 \mid \mathcal{I}_{n+1}}^{-1}\left(A_{n+1}^{1}\right) \oplus\left(\mathcal{I}_{n} \backslash \overline{\mathcal{I}_{n+1}}\right) & \Rightarrow a_{n+2}^{4}=\frac{3}{2} a_{n+1}^{1}+\frac{1}{2} a_{n+1}^{2}+\frac{1}{2} a_{n+1}^{4}
\end{aligned}
$$

Com isso, temos a seguinte relação entre $a_{n+2}^{i}$ e $a_{n}^{i}$ :

$$
\begin{aligned}
& a_{n+2}^{1}=\frac{1}{4}\left(a_{n}^{1}+a_{n}^{2}+2 a_{n}^{4}\right) \\
& a_{n+2}^{2}=\frac{1}{2} a_{n}^{2} \\
& a_{n+2}^{3}=\frac{1}{4}\left(a_{n}^{1}+a_{n}^{2}+2 a_{n}^{3}\right) \\
& a_{n+2}^{4}=\frac{1}{4}\left(a_{n}^{1}+a_{n}^{2}+a_{n}^{3}+3 a_{n}^{4}\right)
\end{aligned}
$$

Temos, então, a seguinte fórmula de recursão:

$$
\left(a_{n+2}^{1}, a_{n+2}^{2}, a_{n+2}^{3}, a_{n+2}^{4}\right)=\left(a_{n}^{1}, a_{n}^{2}, a_{n}^{3}, a_{n}^{4}\right) \mathbf{A}, \quad \text { onde } \mathbf{A}=\frac{1}{4}\left(\begin{array}{cccc}
1 & 0 & 1 & 1 \\
1 & 2 & 1 & 1 \\
0 & 0 & 2 & 1 \\
2 & 0 & 0 & 3
\end{array}\right)
$$

A matriz A tem um auto valor igual a 1 e seu correspondente autovetor $(2,0,1,3)$.

A Proposição 5.2.1 prova que se escolhemos os módulos dos anéis da primeira etapa como sendo um múltiplo do auto vetor $(2,0,1,3)$, então os módulos não decrescem. $\mathrm{O}$ seguinte Lema mostra que de fato, os módulos crescem linearmente em cada etapa da induçào.

Lema 5.2.2 Existe $\epsilon>0$ tal que para todo $n \geq 1$ com $\Phi_{n}$ do tipo $F_{1}$ que satisfaz

$$
a_{2 n+2}^{1} \geq a_{2 n}^{1}+\epsilon, \quad a_{2 n+2}^{4} \geq a_{2 n}^{4}+\epsilon .
$$


Prova: Vamos considerar a aplicação $\Phi_{n+1}$ que aplica $\mathcal{I}_{n+1}$ em $\mathcal{J}_{n}$. Lembre-se que pela mesma aplicação $\tilde{\mathcal{C}}_{n+2}$ é aplicado sobre $\mathcal{J}_{n+2}$. Sejam $U_{2}:=A_{n+1}^{2} \oplus \mathcal{J}_{n+1} \backslash \overline{\mathcal{J}_{n+2}}$ e $W_{2}:=A_{n+1}^{1}$. Sejam também $U_{1}:=A_{n+2}^{3}$ e $W_{1}:=\mathcal{I}_{n+1} \sqrt{\left(A_{n+2}^{3} \oplus \tilde{\mathcal{C}}_{n+2}\right)}$. Verifica-se que $\Phi_{n+1}\left(U_{1}\right)=U_{2}$ e $\Phi_{n+1}\left(W_{1}\right)=W_{2}$, sendo $\Phi_{n+1}$ restrita a $U_{1}$ é univalente, e $\Phi_{n+1}$ restrita. a $W_{1}$ é um recobrimento de grau 2 .

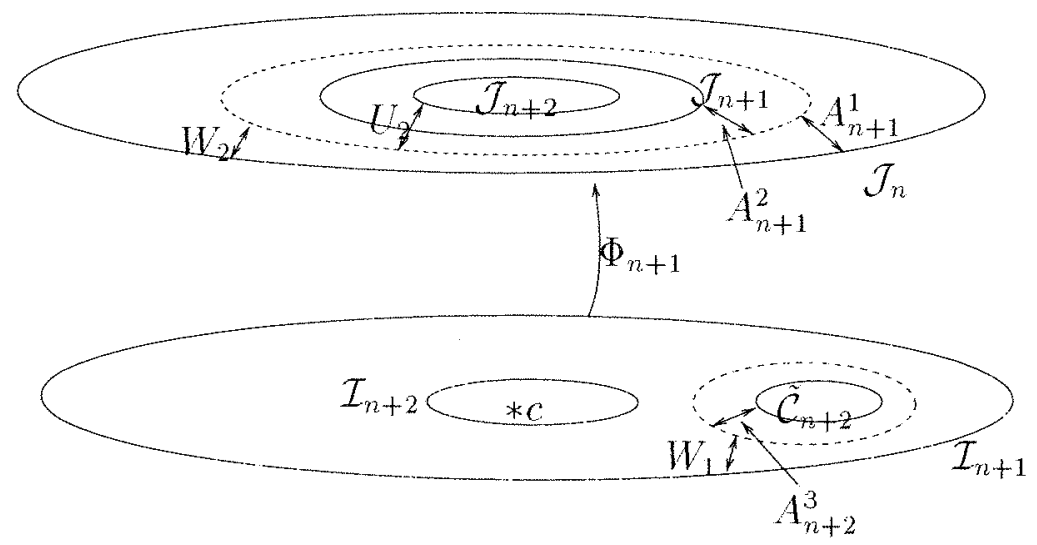

Figura 5.3: Crescimento do módulo no Tipo $F_{1} F_{2}$

Sabemos que $\bmod \left(\mathcal{J}_{n+1} \backslash \overline{\mathcal{J}_{n+2}}\right) \geq \frac{1}{2}\left(\bmod A_{n+1}^{3}+\bmod A_{n+1}^{4}\right)$. Então temos que

$$
\begin{gathered}
\bmod U_{2} \geq \frac{1}{4}\left(a_{n}^{1}+a_{n}^{2}+2 a_{n}^{3}\right), \\
\bmod W_{2}+\bmod U_{2} \geq \frac{1}{4}\left(a_{n}^{1}+a_{n}^{2}+2 a_{n}^{4}\right) .
\end{gathered}
$$

Então pelo Lema 3.2.1 existe uma constante $\epsilon_{n}>0$ tal que

$$
\bmod \left(U_{1} \oplus W_{1}\right) \geq \frac{1}{4}\left(a_{n}^{1}+a_{n}^{2}+a_{n}^{3}+a_{n}^{4}\right)+\epsilon_{n}
$$

Como $A_{n+2}^{4}$ é definido como a união de $W_{1}$ e $\mathcal{I}_{n} \backslash \overline{\mathcal{I}_{n+1}}$ e que $\bmod \left(\mathcal{I}_{n} \backslash \overline{\mathcal{I}_{n+1}}\right) \geq a_{n}^{4} / 2$ resulta-se que

$$
\bmod \left(A_{n+2}^{3} \oplus A_{n+2}^{4}\right) \geq \frac{1}{4}\left(a_{n}^{1}+a_{n}^{2}+a_{n}^{3}+3 a_{n}^{4}\right)+\epsilon_{n}
$$


onde $\epsilon_{n}$ depende de $\delta_{n}$ e tal que $\bmod W_{2} \geq \delta_{n}$. Vamos denotar por $a_{n}$ o vetor $\left(a_{n}^{1}, a_{n}^{2}, a_{n}^{3}, a_{n}^{4}\right)$ e vamos definir $a_{n_{0}}=(\alpha, 0,0,0) \operatorname{com} \alpha>0$. Então aplicando a matriz $\mathbf{A}$ da Proposição 5.2 .1 obtemos

$$
a_{n_{0}+2}=(\alpha / 4,0, \alpha / 4, \alpha / 4)
$$

A presença do auto valor 1 da matriz $\mathbf{A}$ garante que $a_{n}^{1}, a_{n}^{3}$ e $a_{n}^{4}$ estejam afastados de zero sendo maiores que uma constante positiva, digamos, $\delta$. Lembre-se que $W_{2}=\Phi_{n}^{-1}\left(A_{n}^{4}\right)$ e $A_{n}^{4}$ contém $\mathcal{I}_{n-2} \backslash \overline{\mathcal{I}_{n-1}}$. Como

$$
\bmod \left(\mathcal{I}_{n-2} \backslash \overline{\mathcal{I}_{n-1}}\right) \geq \frac{1}{2}\left(\bmod A_{n-2}^{3}+\bmod A_{n-2}^{4}\right)
$$

temos que

$$
\bmod W_{2} \geq \frac{1}{4} a_{n-2}^{4} \geq \frac{1}{4} \delta
$$

Isto implica que existe uma constante $\epsilon>0$ que independe de $n$ tal que $\epsilon \geq \epsilon$ e assim obtemos o aumento dos números $a_{n}^{1}$ e $a_{n}^{4}$ no processo da indução. Isto conclui a prova. 


\subsubsection{Polinômios cúbicos eventualmente Fibonacci do tipo $F_{3} F_{4} F_{5}$}

Assumimos que $\phi_{n_{0}}$ é do tipo $F_{3}$ e pelo Teorema 2.3 .2 teremos que $\phi_{n_{0}+3 i}, \phi_{n_{0}+3 i+1} \mathrm{e}$ $\phi_{n_{0}+3 i+2}$, para $i \geq 0$, são do tipo $F_{3}, F_{4}$ e $F_{5}$, respectivamente. Dado então $\phi_{n}$ do tipo $F_{3}$ temos que $C_{n} \subset I_{n-1}, D_{n} \subset J_{n-1}$ e $\phi_{n}\left(C_{n}\right)=J_{n-1}, \phi_{n}\left(D_{n}\right)=I_{n-1}$. Isto determina que $I_{n}$ é aplicado sobre $I_{n-1}$ e $J_{n}$ sobre $\mathcal{J}_{n-1}$ Agora temos que $\phi_{n+1}$ é do tipo $F_{4}$, ou seja $C_{n+1} \subset J_{n}, D_{n+1} \subset I_{n}$ e $\phi_{n+1}\left(C_{n+1}\right)=J_{n}, \phi_{n}\left(D_{n+1}\right)=I_{n}$. Temos também que $I_{n+1}$ é aplicado sobre $J_{n}$, e $J_{n+1}$ sobre $I_{n}$. Assim temos que $\phi_{n+2}$ é do tipo $F_{5}$, ou seja $C_{n+2} \subset J_{n+1}, D_{n+2} \subset I_{n+1}$ e $\phi_{n+2}\left(C_{n+2}\right)=I_{n+1}, \phi_{n+2}\left(D_{n+2}\right)=J_{n+1}$. Temos assim que $\phi_{n+3}$ é novamente do tipo $F_{3}$.

Proposição 5.2.2 Dada uma aplicação bimodal $f$ do tipo $F_{3} F_{4} F_{5} \ldots$ existe uma seqüência de intervalos $U_{1} \supset U_{2} \supset U_{3} \supset U_{4} \supset \cdots \supset\{c\}$, tal que $U_{n+1}$ é componente do domínio do primeiro retorno a $U_{n}$. Verifica-se também que a aplicaçäo de primeiro retorno de $U_{2 n}$ a $U_{2 n-1}$ é unimodal e a de $U_{2 n+1}$ a $U_{2 n}$ é trimodal, ou seja, com três pontos críticos.

Prova: Vamos considerar a aplicação de primeiro retorno $\phi_{1}$ a $I_{0} \cup J_{0}$ que é do tipo $F_{5}$. Então temos dois domínios críticos $I_{1} \subset I_{0}, J_{1} \subset J_{0}$ e dois domínios pós-críticos $C_{1} \subset J_{0}, D_{1} \subset I_{0}$, sendo que

$$
\begin{aligned}
& \phi_{1}: I_{1} \rightarrow J_{0}, \phi_{1}: J_{1} \rightarrow I_{0} \\
& \phi_{1}: C_{1} \rightarrow I_{0}, \quad \phi_{1}: D_{1} \rightarrow J_{0}
\end{aligned}
$$

Primeiro passo: Seja $U_{1}:=I_{1}$ e definimos em $U_{1}$ uma aplicação de primeiro retorno a $I_{-1}=(0, p)$, à qual chamamo-la $\psi_{1}$. Desta forma temos que $\psi_{1 \mid U_{1}}=f_{\mid J_{0}} \circ \phi_{1 \mid U_{1}}$. Temos então que essa aplicação é trimodal, ou seja, possui três pontos críticos, sendo que dois valores críticos são idênticos e estão contidos em $I_{-1} \backslash \bar{I}_{0}$ e um valor crítico está contido 
em $U_{1}$. Seja $U_{2} \ni c$ o intervalo maximal contido em $U_{1}$ tal que $\psi_{1}\left(U_{2}\right) \subset U_{1}$. Definimos neste domínio $\phi_{2}=\psi_{1}$. A seguir definimos $C_{2}:=\phi_{1}^{-1}\left(J_{1}\right), D_{2}:=\phi_{1}^{-1}\left(U_{1}\right)$ e nestes intervalos definimos $\phi_{2}:=\phi_{1}$. Como $\phi_{1}(d) \in D_{1}$ definimos $J_{2}:=\phi_{1}^{-1}\left(D_{1}\right)$ e $\phi_{2}:=\phi_{1}^{2}$ neste domínio. Sendo assim temos que $C_{2} \subset U_{1}, D_{2} \subset J_{1} \mathrm{e}$

$$
\begin{aligned}
& \phi_{2}: U_{2} \rightarrow U_{1}, \quad \phi_{2}: J_{2} \rightarrow J_{0} \\
& \phi_{2}: C_{2} \rightarrow J_{1}, \quad \phi_{2}: D_{2} \rightarrow U_{1}
\end{aligned}
$$

Verifica-se que $\phi_{2}$ é do tipo $F_{3}$.

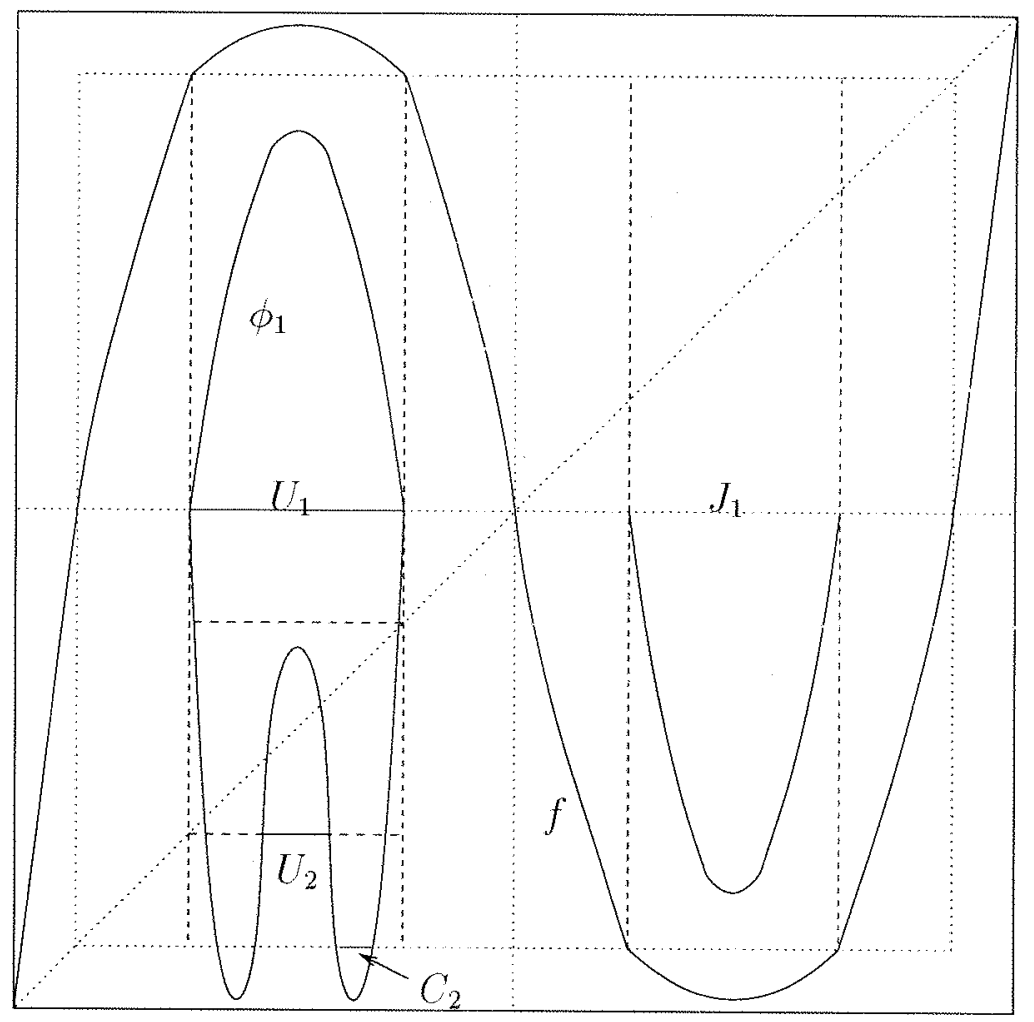

Figura 5.4: Fibonacci Tipo $F_{5}$ e trimodal $\psi_{1}$ definida em $U_{1}$ 
Passo $n$ : Vamos supor que para $3 n-1$ se satisfaz

$$
\begin{gathered}
\phi_{3 n-1}: U_{2 n} \rightarrow U_{2 n-1}, \quad \phi_{3 n-1}: J_{3 n-1} \rightarrow J_{3 n-3} \\
\phi_{3 n-1}: C_{3 n-1} \rightarrow J_{3 n-2}, \quad \phi_{3 n-1}: D_{3 n-1} \rightarrow U_{2 n-1}
\end{gathered}
$$

A seguir construiremos os domínios e a aplicação para o nivel $3 n$. Sabe-se que $\phi_{3 n}(c) \in$ $J_{3 n-1}$ e $\phi_{3 n}(d) \in U_{2 n}$. Então construímos os domínios $C_{3 n}, D_{3 n}$ de modo que $C_{3 n}:=$ $\phi_{3 n-1}^{-1}\left(J_{3 n-1}\right), \quad D_{3 n}:=\phi_{3 n-1}^{-1}\left(U_{2 n}\right)$ e definimos nestes domínios $\phi_{3 n}:=\phi_{3 n-1}$. Sejam também $I_{3 n}:=\phi_{3 n-1}^{-1}\left(C_{3 n-1}\right), J_{3 n}:=\phi_{3 n-1}^{-1}\left(D_{3 n-1}\right)$ e definimos nestes domínios $\phi_{3 n}:=$ $\phi_{3 n-1}^{2}$. Então temos que $C_{3 n} \subset J_{3 n-1}, D_{3 n} \subset U_{2 n}$ e

$$
\begin{aligned}
& \phi_{3 n}: I_{3 n} \rightarrow J_{3 n-2}, \quad \phi_{3 n}: J_{3 n} \rightarrow U_{2 n-1} \\
& \phi_{3 n}: C_{3 n} \rightarrow J_{3 n-1}, \quad \phi_{3 n}: D_{3 n} \rightarrow U_{2 n}
\end{aligned}
$$

Verifica-se que $\phi_{3 n}$ é do tipo $F_{4}$.

Vamos construir agora os domínios e a aplicação para o nível $3 n+1$. Sabemos que $\phi_{3 n+1}(c) \in J_{3 n}$ e $\phi_{3 n+1}(d) \in I_{3 n}$. Então construímos os domínios $C_{3 n+1}, D_{3 n+1}$ de modo que $C_{3 n+1}:=\phi_{3 n}^{-1}\left(I_{3 n}\right), D_{3 n+1}:=\phi_{3 n}^{-1}\left(J_{3 n}\right)$ e definimos nestes dominios $\phi_{3 n+1}:=\phi_{3 n}$. Construímos também $I_{3 n+1}:=\phi_{3 n}^{-1}\left(C_{3 n}\right), J_{3 n+1}:=\phi_{3 n}^{-1}\left(D_{3 n}\right)$ e definimos nestes domínios $\phi_{3 n+1}:=\phi_{3 n}^{2}$. Assim temos que $C_{3 n+1} \subset J_{3 n}, D_{3 n+1} \subset I_{3 n} \mathrm{e}$

$$
\begin{gathered}
\phi_{3 n+1}: I_{3 n+1} \rightarrow J_{3 n-1}, \quad \phi_{3 n+1}: J_{3 n+1} \rightarrow I_{3 n-1} \\
\phi_{3 n+1}: C_{3 n+1} \rightarrow I_{3 n}, \quad \phi_{3 n+1}: D_{3 n+1} \rightarrow J_{3 n}
\end{gathered}
$$

Verifica-se que $\phi_{3 n+1}$ é do tipo $F_{5}$.

Vamos considerar a aplicação $\phi_{3 n}: J_{3 n} \rightarrow U_{2 n-1}$. Temos então um intervalo $\tilde{J}_{3 n}$ tal que $J_{3 n+1} \subset \tilde{J}_{3 n} \subset J_{3 n}$ e $\phi_{3 n}\left(\partial \tilde{J}_{3 n}\right) \subset \partial U_{2 n}$. Daí definimos o intervalo $U_{2 n+1}:=$ $\phi_{3 n+1}^{-1}\left(\tilde{J}_{3 n}\right)$. Definimos neste intervalo $\psi_{2 n+1}:=\phi_{3 n} \circ \phi_{3 n+1}$. Assim temos que $U_{2 n+1}$ é 
o domínio do primeiro retorno a. $U_{2 n}$ e $\psi_{2 n+1}: U_{2 n+1} \rightarrow U_{2 n}$ é uma aplicação trimodal, sendo que dois valores são idênticos e estão contidos em $D_{3 n} \subset U_{2 n}$ e um valor crítico em $U_{2 n+1}$. Seja então $U_{2 n+2} \subset U_{2 n+1}$ o intervalo maximal tal que $\psi_{2 n+1}\left(U_{2 n+2}\right) \subset$ $U_{2 n+1}$. Então definimos neste intervalo $\phi_{3 n+2}:=\psi_{2 n+1}$. A seguir definimos $C_{3 n+2}:=$ $\phi_{3 n+1}^{-1}\left(J_{3 n+1}\right), D_{3 n+2}:=\phi_{3 n+1}^{-1}\left(U_{2 n+1}\right)$ e nestes intervalos definimos $\phi_{3 n+2}:=\phi_{3 n+1}$. Definimos também $J_{3 n+2}:=\phi_{3 n+1}^{-1}\left(D_{3 n+1}\right)$ e $\phi_{3 n+2}:=\phi_{3 n+1}^{2}$ neste intervalo. Sendo assim temos que $C_{3 n+2} \subset U_{2 n+1}, D_{3 n+2} \subset J_{3 n+1} \mathrm{e}$

$$
\begin{aligned}
\phi_{3 n+2}: U_{2(n+1)} & \rightarrow U_{2 n+1}, \quad \phi_{3 n+2}: J_{3 n+2} \rightarrow J_{3 n} \\
\phi_{3 n+2}: C_{3 n+2} & \rightarrow J_{3 n+1}, \quad \phi_{3 n+2}: D_{3 n+2} \rightarrow U_{2 n+1}
\end{aligned}
$$

Verifica-se que $\phi_{3 n+2}$ é do tipo $F_{3}$. Isto satisfaz a hipótese da indução, e podemos continuar a construção. Isto concluí a prova da Proposição.

A seguir vamos mostrar que a seqüência obtida na Proposição 5.2 .2 implica o decaimento exponencial de geometria. Dados então $\phi_{n}, U_{n}, I_{n}, J_{n}, C_{n}, D_{n}$ consideramos a continuação analítica $\Phi_{n}, \mathcal{U}_{n}, \mathcal{I}_{n}, \mathcal{J}_{n}, \mathcal{C}_{n}, \mathcal{D}_{n}$.

Lema 5.2.3 Dada a seqüencia de domínios de primeiro retorno da Proposiçâo 5.2.2, existem constantes $C>0, \lambda>1$ tal que para todo $n>0$,

$$
\bmod \left(\mathcal{U}_{2 n-1} \backslash \overline{\mathcal{U}_{2 n}}\right) \geq C \lambda^{n}
$$

Prova: Como a construção se repete de modo análogo no decorrer da induçào, estimaremos o crescimento dos módulos do nível $n=1$ para $n=2$. Lembre-se que $\mathcal{U}_{2}$ é o domínio do primeiro retorno a $\mathcal{U}_{1}$ e $\mathcal{J}_{2}$ é o domínio do primeiro retorno a $\mathcal{J}_{0}$. Então vamos construir quatro anéis $A_{2}^{i}, i=1,2,3,4$ para $\mathcal{U}_{2}, \mathcal{C}_{2}$ dentro de $\mathcal{U}_{1}$. Vamos construir também quatro anéis $B_{2}^{i}, i=1,2,3,4$ para $\mathcal{J}_{2}, \mathcal{D}_{2}$ dentro de $\mathcal{J}_{0}$. 

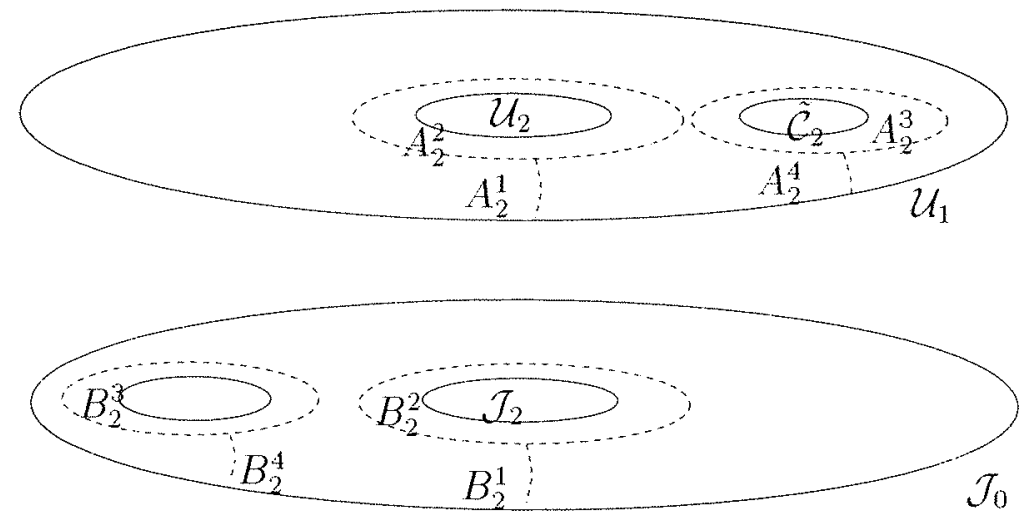

Figura 5.5: 8 anéis do Fibonacci Tipo $F_{3}$

A seguir construimos os anéis para o nível 3 tomando pré-imagens dos anéis do nível 2 pela aplicação $\Phi_{2}$. Como $\mathcal{C}_{3} \subset \mathcal{J}_{2}$ temos anéis $B_{3}^{i}$ para $\mathcal{C}_{3}$

$$
\begin{aligned}
& B_{3}^{2}=\Phi_{2}^{-1}\left(B_{2}^{3}\right) \quad \Rightarrow \quad b_{3}^{2}=\frac{1}{2} b_{2}^{3} \\
& B_{3}^{1}=\Phi_{2}^{-1}\left(B_{2}^{4}\right) \quad \Rightarrow \quad b_{3}^{1}=\frac{1}{2} b_{2}^{4} \\
& B_{3}^{3}=\Phi_{2}^{-1}\left(B_{2}^{2}\right) \quad \Rightarrow \quad b_{3}^{3}=b_{2}^{2} \\
& B_{3}^{4}=\Phi_{2}^{-1}\left(B_{2}^{1}\right) \quad \Rightarrow \quad b_{3}^{4}=\frac{1}{2} b_{2}^{1}
\end{aligned}
$$

Também como $\mathcal{D}_{3} \subset \mathcal{U}_{2}$, definimos os anéis $A_{3}^{i}$ para $\mathcal{D}_{3}$

$$
\begin{aligned}
& A_{3}^{2}=\Phi_{2}^{-1}\left(A_{2}^{3}\right) \quad \Rightarrow \quad a_{3}^{2}=\frac{1}{2} a_{2}^{3} \\
& A_{3}^{1}=\Phi_{2}^{-1}\left(A_{2}^{4}\right) \quad \Rightarrow \quad a_{3}^{1}=\frac{1}{2} a_{2}^{4} \\
& A_{3}^{3}=\Phi_{2}^{-1}\left(A_{2}^{2}\right) \quad \Rightarrow \quad a_{3}^{3}=a_{2}^{2} \\
& A_{3}^{4}=\Phi_{2}^{-1}\left(A_{2}^{1}\right) \Rightarrow a_{3}^{4}=\frac{1}{2} a_{2}^{1} .
\end{aligned}
$$

A aplicação $\Phi_{3}$ aplica $\mathcal{I}_{3}$ em $\mathcal{J}_{1}$ e $\mathcal{J}_{3}$ em $\mathcal{U}_{1}$. Então, definimos os anéis $B_{4}^{i}$ para 
$\mathcal{C}_{4} \subset \mathcal{J}_{3}$ tomando pré-imagens dos anéis $A_{3}^{i}$ e do anel $\mathcal{U}_{1} \backslash \overline{\mathcal{U}_{2}}$. Temos então

$$
\begin{aligned}
B_{4}^{2}=\Phi_{3}^{-1}\left(A_{3}^{3}\right) & \Rightarrow b_{4}^{2}=\frac{1}{2} a_{3}^{3} \\
B_{4}^{1}=\Phi_{3}^{-1}\left(A_{3}^{4} \oplus\left(\mathcal{U}_{1} \backslash \overline{\mathcal{U}_{2}}\right)\right) & \Rightarrow b_{4}^{1}=\frac{1}{2} a_{3}^{4}+\frac{1}{2} a_{2}^{1}+\frac{1}{2} a_{2}^{2} \\
B_{4}^{3}=\Phi_{3}^{-1}\left(A_{3}^{2}\right) & \Rightarrow b_{4}^{3}=a_{3}^{2} \\
B_{4}^{4}=\Phi_{3}^{-1}\left(B_{3}^{1} \oplus\left(\mathcal{U}_{1} \backslash \overline{\mathcal{U}_{2}}\right)\right) & \Rightarrow b_{4}^{4}=\frac{1}{2} a_{3}^{1}+\frac{1}{2} a_{2}^{1}+\frac{1}{2} a_{2}^{2} .
\end{aligned}
$$

Vamos definir também os anéis $A_{4}^{i}$ para $\mathcal{D}_{4}$. Lembre-se que $\mathcal{J}_{1} \backslash \overline{\mathcal{J}_{2}}$ contém pelo menos o anel $B_{2}^{2}$.

$$
\begin{aligned}
A_{4}^{2}=\Phi_{3}^{-1}\left(B_{3}^{3}\right) & \Rightarrow a_{4}^{2}=\frac{1}{2} b_{3}^{3} \\
A_{4}^{1}=\Phi_{3}^{-1}\left(B_{3}^{4} \oplus\left(\mathcal{J}_{1} \backslash \overline{\mathcal{J}_{2}}\right)\right) & \Rightarrow a_{4}^{1}=\frac{1}{2} b_{3}^{4}+\frac{1}{2} b_{2}^{2} \\
A_{4}^{3}=\Phi_{3}^{-1}\left(B_{3}^{2}\right) & \Rightarrow a_{4}^{3}=b_{3}^{2} \\
A_{4}^{4}=\Phi_{3}^{-1}\left(B_{3}^{1} \oplus\left(\mathcal{J}_{1} \backslash \overline{\mathcal{J}_{2}}\right)\right) & \Rightarrow b_{4}^{4}=\frac{1}{2} b_{3}^{1}+\frac{1}{2} b_{2}^{2} .
\end{aligned}
$$

Agora vamos estimar o módulo do anel $\mathcal{I}_{4} \backslash \overline{\mathcal{U}}_{3}$. A aplicação $\Phi_{4}$ aplica este anel sobre $\left(\mathcal{J}_{2} \backslash \overline{\mathcal{J}_{3}}\right) \oplus\left(\mathcal{J}_{3} \backslash \overline{\tilde{J}_{3}}\right)$ e o anel $\mathcal{J}_{3} \backslash \overline{\tilde{\mathcal{J}}_{3}}$ é aplicado por $\Phi_{3}$ sobre $\mathcal{U}_{1} \backslash \overline{\mathcal{U}_{2}}$. Então temos que

$$
\bmod \left(\mathcal{I}_{4} \backslash \overline{\mathcal{U}_{3}}\right) \geq \frac{1}{2} b_{3}^{1}+\frac{1}{2} b_{3}^{2}+\frac{1}{4} a_{2}^{1}+\frac{1}{4} a_{2}^{2} .
$$

A seguir, vamos estimar o módulo dos anéis para o nível 5. Lembre-se que $\Phi_{5}$ aplica $\mathcal{U}_{4}$ em $\mathcal{U}_{3}$ e $\mathcal{J}_{5}$ em $\mathcal{J}_{3}$. Então, consideramos os anéis para $\mathcal{C}_{5}$ dentro de $\mathcal{U}_{3}$ e para $\mathcal{D}_{5}$ dentro de $\mathcal{J}_{3}$. Assim temos anéis para $\mathcal{C}_{5}$ que

$$
\begin{aligned}
A_{5}^{2}=\Psi_{4}^{-1}\left(\mathcal{I}_{3} \backslash \overline{\mathcal{U}_{3}}\right) & \Rightarrow a_{5}^{2}=\frac{1}{4} b_{3}^{1}+\frac{1}{4} b_{3}^{2}+\frac{1}{8} a_{2}^{1}+\frac{1}{8} a_{2}^{2}+\frac{1}{2} a_{4}^{1}+\frac{1}{2} a_{4}^{2} \\
A_{5}^{1}=\Psi_{4}^{-1}\left(\mathcal{U}_{2} \backslash \overline{\mathcal{I}_{3}}\right) & \Rightarrow a_{5}^{1}=\frac{1}{2} a_{3}^{2}+\frac{1}{4} a_{3}^{1} \\
A_{5}^{3}=\Psi_{4}^{-1}\left(A_{3}^{3}\right) & \Rightarrow a_{5}^{3}=\frac{1}{2} a_{3}^{3} \\
A_{5}^{4}=\Psi_{4}^{-1}\left(A_{3}^{4}\right) & \Rightarrow a_{5}^{4}=\frac{1}{4} a_{3}^{4} .
\end{aligned}
$$


Temos também anéis para $\mathcal{D}_{5} \subset \mathcal{J}_{3}$

$$
\begin{aligned}
B_{5}^{2}=\Phi_{4}^{-1}\left(A_{4}^{3} \oplus A_{4}^{4}\right) & \Rightarrow b_{5}^{2}=\frac{1}{2} a_{4}^{3}+\frac{1}{2} a_{4}^{4} \\
B_{5}^{1}=\Phi_{4}^{-1}\left(\mathcal{U}_{2} \backslash \overline{\mathcal{I}_{3}}\right) \oplus\left(\mathcal{J}_{3} \backslash \overline{\mathcal{J}_{4}}\right) & \Rightarrow b_{5}^{1}=\frac{1}{2} a_{3}^{1}+\frac{1}{2} a_{3}^{2}+b_{4}^{1}+b_{4}^{2} \\
B_{5}^{3}=\Phi_{4}^{-1}\left(A_{4}^{2} \oplus\left(\mathcal{I}_{4} \backslash \overline{\mathcal{U}_{3}}\right)\right) & \Rightarrow b_{5}^{3}=a_{4}^{2}+\frac{1}{2} b_{3}^{1}+\frac{1}{2} b_{3}^{2}+\frac{1}{4} a_{2}^{1}+\frac{1}{4} a_{2}^{2} \\
B_{5}^{4}=\Phi_{4}^{-1}\left(A_{4}^{1} \oplus\left(\mathcal{U}_{2} \backslash \overline{\mathcal{I}_{3}}\right)\right) \oplus\left(\mathcal{J}_{3} \backslash \overline{\mathcal{J}_{4}}\right) & \Rightarrow b_{5}^{4}=\frac{1}{2} a_{4}^{1}+\frac{1}{2} a_{3}^{1}+\frac{1}{2} a_{3}^{2}+b_{4}^{1}+b_{4}^{2} .
\end{aligned}
$$

Isto com os resultados anteriores temos que

$$
\begin{aligned}
& a_{5}^{1}=\frac{1}{4} a_{2}^{3}+\frac{1}{8} a_{2}^{4} \\
& a_{5}^{2}=\frac{1}{8} a_{2}^{1}+\frac{1}{8} a_{2}^{2}+\frac{1}{8} b_{2}^{1}+\frac{1}{2} b_{2}^{2}+\frac{1}{8} b_{2}^{3}+\frac{1}{8} b_{2}^{4} \\
& a_{5}^{3}=\frac{1}{2} a_{2}^{2} \\
& a_{5}^{4}=\frac{1}{8} a_{2}^{1} \\
& b_{5}^{1}=\frac{3}{4} a_{2}^{1}+a_{2}^{2}+\frac{1}{4} a_{2}^{3}+\frac{1}{4} a_{2}^{4} \\
& b_{5}^{2}=\frac{1}{4} b_{2}^{2}+\frac{1}{4} b_{2}^{3}+\frac{1}{8} b_{2}^{4} \\
& b_{5}^{3}=\frac{1}{4} a_{2}^{1}+\frac{1}{4} a_{2}^{2}+\frac{1}{2} b_{2}^{2}+\frac{1}{4} b_{2}^{3}+\frac{1}{4} b_{2}^{4} \\
& b_{5}^{4}=\frac{3}{4} a_{2}^{1}+a_{2}^{2}+\frac{1}{4} a_{2}^{3}+\frac{1}{4} a_{2}^{4}+\frac{1}{8} b_{2}^{1}+\frac{1}{4} b_{2}^{2} .
\end{aligned}
$$

Então, podemos escrever esta relação do seguinte modo:

$$
\left(a_{5}^{1}, a_{5}^{2}, a_{5}^{3}, a_{5}^{4}, b_{5}^{1}, b_{5}^{2}, b_{5}^{3}, b_{5}^{4}\right)=\left(a_{2}^{1}, a_{2}^{2}, a_{2}^{3}, a_{2}^{4}, b_{2}^{1}, b_{2}^{2}, b_{2}^{3}, b_{2}^{4}\right) \mathbf{B}
$$




$$
\text { onde } \mathbf{B}=\frac{1}{8}\left(\begin{array}{cccccccc}
0 & 1 & 0 & 1 & 6 & 0 & 2 & 6 \\
0 & 1 & 4 & 0 & 8 & 0 & 2 & 8 \\
2 & 0 & 0 & 0 & 2 & 0 & 0 & 2 \\
1 & 0 & 0 & 0 & 2 & 0 & 0 & 2 \\
0 & 1 & 0 & 0 & 0 & 0 & 0 & 1 \\
0 & 4 & 0 & 0 & 0 & 2 & 4 & 2 \\
0 & 1 & 0 & 0 & 0 & 2 & 2 & 0 \\
0 & 1 & 0 & 0 & 0 & 1 & 2 & 0
\end{array}\right)
$$

O polinômio característica desta matriz é:

$$
p_{B}(\lambda)=\lambda^{8}-5 \lambda^{7}-21 \lambda^{6}-21 \lambda^{5}-52 \lambda^{4}-14 \lambda^{3}+8 \lambda^{2} .
$$

Verifica-se, então, que a matriz obtida possui um autovalor maior do que 1 e um autovetor com componentes todas positivas.

Lmebre-se que $C_{2}=\phi_{1}^{-1}\left(J_{1}\right)$ e $f\left(J_{1}\right)$ está contido no complementar de $I_{0}$, ou seja $\psi_{1}\left(C_{2}\right)$ está contido no complementar de $I_{0}$, enquanto $\phi_{2}\left(U_{2}\right) \subset U_{1}$. Isto implica que $U_{2}$ e $C_{2}$ estão afastados entre si por um intervalo com comprimento positivo e que $C_{2}$ está afastado do bordo de $U_{1}$ por uma distância positiva. Este fato nos permite tomar os módulos dos anéis iniciais $A_{2}^{i}$ de modo que:

$$
\left(a_{2}^{1}, a_{2}^{2}, a_{2}^{3}, a_{2}^{4}\right)=(\alpha, \alpha, \alpha, \alpha)
$$

onde $\alpha>0$. Lembre-se também que os anéis $B_{2}^{i}$ são definidos no anel $\mathcal{J}_{0} \backslash \overline{\mathcal{J}_{2}}$ tendo $\mathcal{D}_{2} \subset \mathcal{J}_{1}$. Como o anel $\mathcal{J}_{0} \backslash \overline{\mathcal{J}_{1}}$ tem módulo positivo, podemos escolher $\left(b_{2}^{1}, b_{2}^{2}, b_{2}^{3}, b_{2}^{4}\right)=$ 
$(\alpha, 0,0, \alpha)$. Aplicando a matriz adquirida na proposição obtemos que

$$
\left(a_{5}^{1}, a_{5}^{2}, a_{5}^{3}, a_{5}^{4}, b_{5}^{1}, b_{5}^{2}, b_{5}^{3}, b_{5}^{4}\right)=\frac{1}{8}(3 \alpha, 4 \alpha, 4 \alpha, \alpha, 18 \alpha, \alpha, 6 \alpha, 19 \alpha)
$$

sendo assim todas as componentes positivas. Podemos então escolher os módulos dos anéis do nível 5 como sendo um múltiplo de autovetor da matriz $\mathbf{B}$. Aplicando a matriz a este vetor, por indução, resulta-se que $\bmod \left(\mathcal{U}_{2 n-1} \backslash \overline{\mathcal{U}_{2 n}}\right)$ cresce exponencialmente, concluindo assim a prova da Proposição.

Lembre-se que existe uma seqüência de domínios do primeiro retorno definido como sendo a seqüência das peças de Yoccoz $P_{0}(c) \supset P_{m_{1}}(c) \supset P_{m_{2}}(c) \supset \ldots$ e $P_{0}(d) \supset$ $P_{n_{1}}(d) \supset P_{n_{2}}(d) \supset \ldots$ Mostraremos na Proposição seguinte que a seqüência $U_{n}$ obtida na Proposiçào 5.2 .2 está bem encaixada na partição de Yoccoz.

Proposição 5.2.3 Para todo $k \geq 1$ verifica-se que

$$
U_{2 k-1}=P_{m_{3 k-2}}(c) e U_{2 k}=P_{m \imath_{3 k-1}}(c) \text {. }
$$

Prova: Dado o ponto fixo $p$ da aplicação $f$, inicialmente temos por definição $P_{0}(c)=\left(p_{c}, p\right)=I_{0}$ e $P_{0}(d)=\left(p, p_{d}\right)=J_{0}$, onde $p_{c}, p_{d}$ são pré-imagens de $p$ por $f$. A seguir $P_{m_{1}}(c), U_{1}$ e $P_{n_{1}}(d), J_{1}$ foram definidos da mesma maneira como sendo domínios críticos de primeiro retorno a $I_{0} \cup J_{0}$. Verifica-se então que

$$
P_{m_{1}}(c)=U_{1} \text { e } P_{n_{1}}(d)=J_{1}
$$

Temos também domínios pós-críticos $C_{1}=f^{-1}\left(I_{0}\right)=C_{m_{1}}$ e $D_{1}=f^{-1}\left(J_{0}\right)=D_{n_{1}}$. 
Primeiro Passo: Observamos os domínios do nível 2. $P_{m_{2}}(c)$ é o domínio maximal de primeiro retorno a $P_{m_{1}}$ por uma aplicação unimodal e é definido por $P_{m_{2}}(c)=$ $\phi_{n_{1}}^{-1}\left(\tilde{C}_{m_{1}}\right)$. O mesmo verifica-se para $U_{2}$. Temos assim que

$$
P_{m_{2}}=U_{2}
$$

Temos também os domínios $P_{n_{2}}$ e $J_{2}$ definidos por $P_{n_{2}}(d)=\phi_{m_{1}}^{-1}\left(\tilde{D}_{n_{1}}\right), J_{2}=\phi_{1}^{-1}\left(D_{1}\right)$. Como $\tilde{D}_{n_{1}} \subset D_{1}$ verifica-se então que

$$
P_{n_{2}}(d) \subset J_{2}
$$

Temos também $C_{m_{2}}=\phi_{m_{1}}^{-1}\left(P_{n_{1}}(d)\right)=\phi_{1}^{-1}\left(J_{1}\right)=C_{2}$ e $D_{n_{2}}=\phi_{n_{1}}^{-1}\left(P_{m_{1}}(c)\right)=\phi_{1}^{-1}\left(U_{1}\right)=$ $D_{2}$.

Passo indutivo: Vamos supor que para $k \geq 1$ verifica-se o seguinte fato:

$$
\begin{gathered}
P_{m_{3 k-1}}(c)=U_{2 k}, \quad P_{n_{3 k-1}}(d) \subset J_{3 k-1}, \\
C_{m_{3 k-1}} \subset C_{3 k-1}, \quad D_{n_{3 k-1}} \subset D_{3 k-1} .
\end{gathered}
$$

A seguir observamos os domínios do nível $3 k$ definidos por $P_{m_{3 k}}(c)=\phi_{m_{3 k-1}}^{-1}\left(\tilde{C}_{m_{z k-1}}\right)$, $I_{3 k}=\phi_{3 k-1}^{-1}\left(C_{3 k-1}\right)$, e $P_{n_{3 k}}(d)=\phi_{3 k-1}^{-1}\left(\tilde{D}_{n_{3 k-1}}\right), J_{3 k}=\phi_{3 k-1}^{-1}\left(D_{3 k-1}\right)$. Verifica-se então que:

$$
P_{m_{3 k}}(c) \subset I_{3 k} \text { e } P_{n_{3 k}}(d) \subset J_{3 k}
$$

Temos também os dominios pós-críticos definidos por $C_{3 k}=\phi_{3 k-1}^{-1}\left(J_{3 k-1}\right), C_{m_{3 k}}=$ $\phi_{n_{3 k-1}}^{-1}\left(P_{n_{3 k-1}}(d)\right)$ e $D_{3 k}=\phi_{3 k-1}^{-1}\left(I_{3 k-1}\right), D_{n_{2 k}}=\phi_{m_{3 k-1}}^{-1}\left(P_{m_{3 k-1}}(c)\right)$. Verifica-se entào que $C_{3 k} \supset C_{m_{3 k}}$ e $D_{3 k}=D_{n_{3 k}}$.

Vamos observar agora os domínios do nível $3 k+1$ definidos por $P_{m_{3 k+1}}(c)=\phi_{m_{3 k}}^{-1}\left(\tilde{C}_{m_{3 k}}\right)$, $I_{3 k+1}=\phi_{3 k}^{-1}\left(C_{3 k}\right)$ e $P_{n_{3 k+1}}(d)=\phi_{n_{3 k}}^{-1}\left(\tilde{D}_{n_{3 k}}\right), J_{3 k+1}=\phi_{3 k}^{-1}\left(D_{3 k}\right)$. Verifica-se então que

$$
P_{m_{3 k+1}}(c) \subset I_{3 k+1}, P_{n_{3 k+1}}(d) \subset J_{3 k+1}
$$


Temos também os domínios pós-críticos $C_{3 k+1}=\phi_{3 k}^{-1}\left(I_{3 k}\right), C_{m_{3 k+1}}=\phi_{n_{3 k}}^{-1}\left(P_{m_{3 k}}(c)\right) \mathrm{e}$ $D_{3 k+1}=\phi_{3 k}^{-1}\left(J_{3 k}\right), D_{n_{3 k+1}}=\phi_{m_{3 k}}^{-1}\left(P_{n_{3 k}}(d)\right)$. Na Proposição 5.2.2, observamos que $U_{2 k+1}$ foi definido de modo que

$$
\phi_{3 k \mid J_{3 k}} \circ \phi_{3 k+1 \mid I_{3 k+1}}\left(\partial U_{2 k+1}\right) \subset \partial U_{2 k}
$$

e que essa aplicação é uma trimodal, sendo aplicação de primeiro retorno de $U_{2 k+1}$ a $U_{2 k}$. Entretanto, para $P_{m_{3 k+1}}(c)$ temos que

$$
\phi_{n_{3 k} \mid P_{n_{3 k}}(d)} \circ \phi_{m_{3 k+1} \mid P_{m_{3 k+1}}(c)}\left(\partial P_{m_{3 k+1}}(c)\right) \subset \partial P_{m_{3 k-1}}(c) .
$$

Observamos que esta é a aplicação de primeiro retorno de $P_{m_{3 k+1}}(c)$ a $P_{m_{3 k-1}}(c)$ como uma aplicação trimodal. Como $U_{2 k}=P_{m_{3 k-1}}, \phi_{n_{3 k} \mid P_{n_{3 k}}(d)=\phi_{3 k \mid J} J_{3 k}}$ e $\phi_{m_{3 k+1} \mid P_{m_{3 k+1}}(c)}=$ $\phi_{3 k+1 \mid I_{3 k+1}}$, temos que

$$
P_{m_{3 k+1}}(c)=U_{2 k+1}
$$

Sabemos que $P_{m_{3 k+2}}(c)$ é o domínio de primeiro retorno a $P_{m_{3 k+1}}(c)$, ou seja, $P_{m_{2 k+2}}(c)=$ $\phi_{m_{3 k+1}}^{-1}\left(P_{m_{3 k+1}}(c)\right)$, e $U_{2 k+2}$ foi definido como sendo o domínio maximal contido em $U_{2 k+1}$ tal que $\psi_{2 k+1}\left(U_{2 k+2}\right) \subset U_{2 k+1}$, ou seja, domínio crítico do primeiro retorno a $U_{2 k+1}$. Estas construşôes implicam que

$$
P_{m_{3 k+2}}=U_{2 k+2} \text { e } P_{n_{3 k+2}}(d) \subset J_{3 k+2} .
$$

Temos também os domínios pós-críticos correspondentes satisfazendo $C_{m_{3 k+2}} \subset C_{3 k+2}$, $D_{n_{3 k+2}} \subset D_{3 k+2}$, satisfazendo assim a condição da indução. Isto conclui a prova da Proposição.

O resultado da Proposição 5.2.3 com o Lema 5.2.3 prova que $\frac{\left|P_{m_{3 k+2}}\right|}{\left|P_{m_{3 k+1}}\right|}$ decai exponencialmente. 


\section{Capítulo 6}

\section{Hiperbolicidade Induzida}

\subsection{Existência da Aplicação Induzida}

Nesta seção mostraremos que uma aplicação $f$ bimodal sem retornos centrais induz uma aplicaçào $\mathcal{F}$ de tal forma que $\mathcal{F}$ restrita a cada componente conexa do seu domínio é monótona e a imagem de $\mathcal{F}$ é união finita de intervalos. Exigiremos ainda que todos os ramos de $\mathcal{F}^{n}, n=1,2, \cdots$, tenham distorção uniformemente limitada. A partir desta aplicação induzida mostraremos mais tarde a hiperbolicidade da aplicação.

Proposição 6.1.1 Dada $f$ uma aplicaçâo bimodal sem retornos centrais existem intervalos $I_{0}, J_{0}$ e uma família $\left\{\Delta_{i}\right\}$ enumerável (possivelmente finita) de intervalos abertos dois a dois disjuntos. Existem também uma aplicação $\mathcal{F}$ e uma constante a tal que:

1. $\left(I_{0} \cup J_{0}\right) \backslash \cup_{i} \Delta_{i}$ é um conjunto de Cantor.

2. Para cada $i$, existe $n_{i}$ tal que $\mathcal{F}_{\mid \Delta_{i}}=f_{\mid \Delta_{i}}^{n_{i}}$. 
3. Para cada $\Delta_{i}$, aplicaçấo $\mathcal{F}$ restrita a $\Delta_{i}$ é monótona sobre $I_{0}$ ou $J_{0}$.

4. $\mathcal{F}_{\mid \Delta_{i}}=f_{\mid \Delta_{i}}^{n_{1}}$ é $\alpha$-extensível (isto é: cada $\Delta_{i}$ possui uma vizinhança $\hat{\Delta}_{i}$ tal que $f^{n_{i}}\left(\hat{\Delta}_{i}\right)$ é uma a-vizinhança de $f^{n_{i}}\left(\Delta_{i}\right)$ ).

A propriedade do intervalo $\hat{\Delta}$ no item 4 da Proposição implica o seguinte resultado:

Corolário 6.1.1 Dada $f$ uma aplicação bimodal sem retornos centrais existem intervalos $I_{0}, J_{0}$ e uma familia $\left\{\Delta_{i}\right\}$ enumerável (possivelmente finita) de intervalos abertos dois a dois disjuntos. Existem também uma aplicaçâo $\mathcal{F}$ e uma constante a tal que, para todo $k \geq 1, \mathcal{F}^{k}$ restrita a cada componente conexa do seu domínio é $\alpha$-extensível.

Prova: Sejam $i, j$ tais que $f^{n j}\left(\Delta_{j}\right) \supset \Delta_{i}$. Vamos assumir que $\Delta_{i} \subset I_{0}$. Então, pela Proposiçào 6.1.1, temos que $f^{n_{j}}\left(\hat{\Delta}_{j}\right)$ é uma $\alpha$-vizinhança de $I_{0}$. Seja $\Delta_{j i}=\Delta_{j} \cap f^{-n_{3}}\left(\Delta_{i}\right)$. Definimos $\hat{\Delta}_{j i}:=\hat{\Delta}_{j} \cap f^{-n_{i}}\left(\hat{\Delta}_{i}\right)$. Como $\hat{\Delta}_{i}$ está contido em uma $\tau_{0}$-vizinhança de $I_{0}$, temos que $\hat{\Delta}_{j i} \subset \hat{\Delta}_{j}$ e $f^{n_{j}+n_{i}}$ aplica $\hat{\Delta}_{j i}$ sobre uma $\alpha$-vizinhança de $I_{0}$ ou $J_{0}$. Como esta situação se satisfaz para quaiquer $i, j$ tal que $f^{n_{j}}\left(\Delta_{j}\right) \supset \Delta_{i}$, implica-se que $\mathcal{F}^{2}$ é $\alpha$-extensível. Semelhantemente, para todo $k \geq 1$, os ramos de $\mathcal{F}^{k}$ são $\alpha$-extensiveis.

\section{Prova da Propisição 6.1.1}

\section{Construção inicial}

Dada a aplicação bimodal $f$ com pontos críticos $c, d(c<d)$ e ponto fixo $q$, definimos dois intervalos $I_{0}=\left(q_{c}, q\right)$ e $J_{0}=\left(q, q_{d}\right)$, onde $q_{c}, q_{d}\left(q_{c}<q_{d}\right)$ săo pré-imagens de $q$ por f. Consideramos a aplicação do Primeiro Retorno $\mathcal{F}_{1}$ induzida por $f$ em $I_{0} \cup J_{0}$. Pela hipótese $f(c), f(d)$ não estão contidos em $I_{0} \cup J_{0}$, então sejam $m_{0}$ e $n_{0}$ números mínimos de inteiros positivos tais que $f^{m_{0}}(c) \in I_{0} \cup J_{0}$ e $f^{n_{0}}(d) \in I_{0} \cup J_{0}$. Então podemos ver 
facilmente que $\mathcal{F}_{1}$ possui $2\left(m_{0}-1\right)+2\left(n_{0}-1\right)$ ramos monótonos e dois ramos críticos. Usaremos a notação $I_{1}$ para o domínio do ramo crítico que contém $c, J_{1}$ para o domínio do ramo crítico contendo $d$ e $\Delta_{i}$ para os domínios dos ramos monótonos. Chamaremos de $\phi_{1}$ aos ramos críticos definidos em $I_{1} \cup J_{1}$. Desta forma podemos definir uma partição $\xi_{1}$ de $I_{0} \cup J_{0}$ por

$$
\xi_{1}: I_{0} \cup J_{0}=(\cup \Delta) \cup I_{1} \cup J_{1}
$$

e temos

$$
\mathcal{F}_{1}=\left\{\begin{array}{c}
f^{n_{i}}: \Delta_{i} \rightarrow I_{0} \text { ou } J_{0} \\
\phi_{1}: I_{1} \cup J_{1} \rightarrow I_{0} \cup J_{0}
\end{array}\right.
$$

onde $n_{i}$ é um número inteiro tal que $\mathcal{F}_{1 \mid \Delta_{i}}=f^{n_{i}}$.

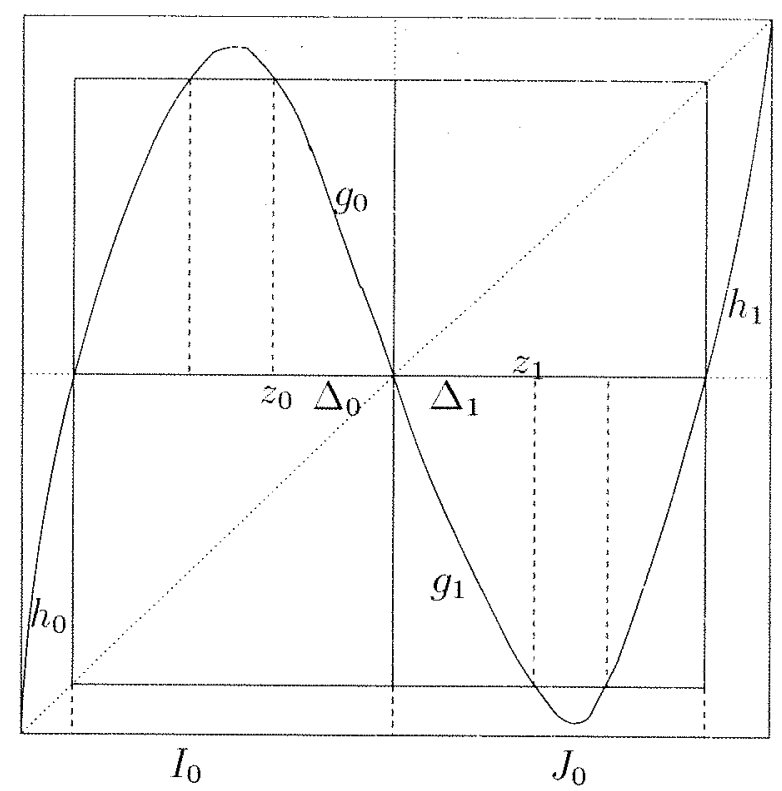

Figura 6.1:

Agora, vamos considerar a extendibilidade de cada ramo de $\mathcal{F}_{1}$. 


\section{Extendibilidade:}

Sejam $\Delta_{0}, \Delta_{0}^{\prime}$ dois intervalos externos da partição $\xi_{1}$ contidos em $I_{0}$ e $\Delta_{1}, \Delta_{1}^{\prime}$, contidos em $J_{0}$. Sejam $h_{0}:=f_{\left[0, q_{c}\right]}$ e $h_{1}:=f_{i\left[q_{d}, 1\right]}$. Assumiremos que $\Delta_{0}$ e $\Delta_{1}$ têm $q$ (ponto fixo) no seu bordo, e sejam $g_{0}:=f_{\mid \Delta_{0}}$ e $g_{1}:=f_{\mid \Delta_{1}}$. Seja $z_{0}(\neq q)$ o bordo de $\Delta_{0}$ e $z_{1}(\neq q)$ o bordo de $\Delta_{1}$. Consideramos uma seqüencia de pré-imagens de $\left(z_{0}, z_{1}\right)$ definida para $n=0,1,2 \ldots$

$$
\left(z_{0}^{n}, z_{1}^{n}\right)=\left(\left(g_{0}^{-1} \circ g_{1}^{-1}\right)^{n}\left(z_{0}\right), \quad\left(g_{1}^{-1} \circ g_{0}^{-1}\right)^{n}\left(z_{1}\right)\right)
$$

Note que $\left(z_{0}^{n}, z_{1}^{n}\right)$ converge a $q$, pois $q$ é um ponto fixo repulsor. Temos então o número mínimo $\tau$ tal que, $h_{0}^{-1}\left(z_{0}^{\tau}\right) \in\left(f^{n_{0}-1}(d), q_{c}\right)$ e $h_{1}^{-1}\left(z_{1}^{\tau}\right) \in\left(q_{d}, f^{m_{0}-1}(c)\right)$. Definimos então

$$
\hat{I}=\left(h_{0}^{-1}\left(z_{0}^{\tau}\right), g_{1}^{-1}\left(z_{0}^{\tau}\right)\right) \quad \text { e } \quad \hat{J}=\left(g_{0}^{-1}\left(z_{1}^{\tau}\right), h_{1}^{-1}\left(z_{1}^{\tau}\right)\right)
$$

Vamos escolher $\alpha$ de tal modo que $\hat{I}$ é uma $\alpha$-vizinhança de $I_{0}$ e $\hat{J}$ é uma $\alpha$-vizinhança de $J_{0}$.

Podemos verificar facilmente que todos os ramos de $\mathcal{F}_{1}$ são $\alpha$-extensíveis. Ainda mais, como $z_{0}^{\tau}<g_{0}^{-1}\left(z_{1}^{\tau}\right)$ e $g_{1}$ é uma aplicação decrescente, temos que $g_{1}^{-1}\left(z_{0}^{\tau}\right)>g_{1}^{-1} \circ g_{0}^{-1}\left(z_{1}^{\tau}\right)$. Isto implica que $\hat{\Delta}_{0} \subset \hat{I}$. E como $h_{0}$ é crescente, temos que $h_{0}^{-1}\left(z_{0}^{\tau}\right)<h_{0}^{-1} \circ g^{-1}\left(z_{1}^{\tau}\right)$. Isto implica que $\hat{\Delta}_{0}^{\prime} \subset \hat{I}$. Analogamente, podemos mostrar que $\hat{\Delta}_{1}$ e $\hat{\Delta}_{1}^{\prime}$ também são contidos em $\hat{J}$. Além disso a extensão de $I_{1}, J_{1}$ está contida em $I_{0}, J_{0}$ respectivamente.

\section{Primeiro Passo}

\section{Dividindo $I_{1} \cup J_{1}$}

Pela hipótese, $\phi_{1}(c), \phi_{1}(d) \notin I_{1} \cup J_{1}$ e temos dois domínios $\Delta_{*}, \Delta_{*}^{\prime}$ de $\xi_{1}$ que contêm $\phi_{1}(c), \phi_{1}(d)$ respectivamente. Denotemos por $f_{*}, f_{*}^{\prime}$ ramos monótonos definidos em $\Delta_{*}, \Delta_{*}^{\prime}$. Refinamos $I_{1}$ e $J_{1}$ usando a pré-imagem de intervalos de $\xi_{1}$ por $\phi_{1}$. Assim definimos uma 
partição em $I_{1} \cup J_{1}$ por:

$$
\eta_{2}^{\prime}: I_{1} \cup J_{1}=(\cup \Delta) \cup\left(\cup I_{1}^{-k}\right) \cup\left(\cup J_{1}^{-k}\right) \cup I_{2} \cup J_{2}
$$

onde $I_{1}^{-k}, J_{1}^{-k}$ são pré-imagens de $I_{1}$ ou de $J_{1}$ por $\phi_{1}$. E $I_{2}$ e $J_{2}$ são domínios dos ramos críticos contendo $c$ e $d$ respectivamente e definidos de modo que $I_{2}=\phi_{1}^{-1}\left(\Delta_{*}\right) \mathrm{e}$ $J_{2}=\phi_{1}^{-1}\left(\Delta_{*}^{\prime}\right)$. As aplicações em cada intervalo de $\eta_{2}^{\prime}$ são definidos por:

$$
\mathcal{F}_{2}^{\prime}=\left\{\begin{array}{c}
\phi_{2}=f_{*} \circ \phi_{1}: I_{2} \rightarrow I_{0} \cup J_{0} \\
\phi_{2}=f_{*}^{\prime} \circ \phi_{1}: J_{2} \rightarrow I_{0} \cup J_{0} \\
f_{\Delta_{i}}=\mathcal{F}_{1} \circ \phi_{1}: \Delta_{i} \rightarrow I_{0} \cup J_{0} \\
\chi: I_{1}^{-k} \rightarrow I_{1}, \quad \chi: J_{1}^{-k} \rightarrow J_{1},
\end{array}\right.
$$

onde $\chi$ é uma aplicação composta de $f$ que leva $I_{1}^{-k}$ (respectivamente $J_{1}^{-k}$ ) difeomorficamente sobre $I_{1}$ (respectivamente $J_{1}$ ).

\section{Extendibilidade:}

Sabemos que os ramos críticos $\phi_{1}$ possuem uma extensão cuja imagem se estende até ao bordo de $\hat{I}$ ou $\hat{J}$, e que todas as componentes conexas do domínio de $\mathcal{F}_{1}$ possuem sua extensão contida em $\hat{I}$ ou $\hat{J}$. Fácil ver que os ramos críticos $\phi_{2}$ definidos em $I_{2} \cup J_{2}$ possuem uma extensão cuja imagem se estende até ao bordo de $\hat{I}$ ou de $\hat{J}$. Denotemos por $\hat{I}_{2} \supset I_{2}$ e $\hat{J}_{2} \supset J_{2}$ os intervalos onde a extensão de $\phi_{2}$ está definida. Seja $\Delta \in \xi_{1}$ tal que $\hat{\Delta}$ esteja contido em $\phi_{1}\left(I_{1} \cup J_{1}\right)$. Então temos que a restrição de $\mathcal{F}_{2}^{\prime} \operatorname{em} \phi_{1}^{-1}(\Delta)$ é $\alpha$-extensivel. Entretanto, podemos ter alguns intervalos $\Delta^{\prime}$ tal que $\Delta^{\prime} \subset \phi_{1}\left(I_{1} \cup J_{1}\right)$, mas $\hat{\Delta}^{\prime} \ni \phi_{1}(c)$ (ou $\phi_{1}(d)$ ) (veja a figura 6.2 ). Neste caso $\mathcal{F}_{2}^{\prime}$ restrita a pré-imagem de $\Delta^{\prime}$ por $\phi_{1}$ não pode ser $\alpha$-extensível. Neste caso, refinaremos $\Delta^{\prime}$ usando o chamado Refinamento no Bordo, isto é: 


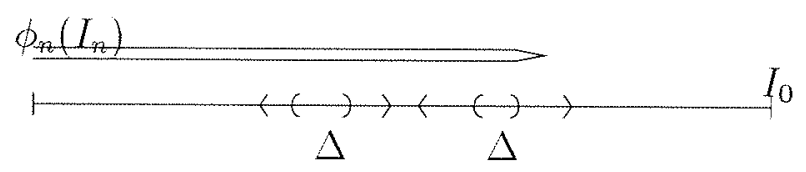

Figura 6.2: $\Delta$-sem extendibilidade

\section{Refinamento no Bordo:}

Vamos refinar $\Delta^{\prime}$ usando a pré-imagem de intervalos de $\xi_{1}$ por $f_{\Delta^{\prime}}$ e temos uma partição em $\Delta^{\prime}$ :

$$
\Delta^{\prime}=(\cup \Delta) \cup\left(\cup I_{1}^{-1}\right) \cup\left(\cup J_{1}^{-1}\right)
$$

Note que os dois ramos externos definidos na prtição em $\Delta^{\prime}$ são monótonos. Então refinamos novamente os domínios destes dois ramos externos usando a pré-imagem de intervalos de $\xi_{1}$ pela aplicação monótona definida netes domínios. Repetimos este processo nos domínios externos da próxima etapa. Repetindo este processo infinitas vezes, obtemos uma partição em $\Delta^{\prime}$ :

$$
\Delta^{\prime}=(\cup \Delta) \cup\left(\cup I_{1}^{-k}\right) \cup\left(\cup J_{1}^{-k}\right) \cup C
$$

onde $C$ é um conjunto de Cantor definido por:

$$
C=\left(\cap_{i \geq 0} \cup_{k \geq i} I_{1}^{-k}\right) \cup\left(\cap_{j \geq 0} \cup_{l \geq j} J_{1}^{-k}\right)
$$

Assim todos os intervalos definidos em $\Delta^{\prime}$ possuem suas extensões contidas na imagem de $\phi_{1}$. Aplicando a pré-imagem dessa partição por $\phi_{1}$, completamos o primeiro refinamento em $I_{1} \cup J_{1}$ :

$$
\eta_{2}^{\prime \prime}: I_{1} \cup J_{1}=(\cup \Delta) \cup\left(\cup I_{1}^{-k}\right) \cup\left(\cup J_{1}^{-k}\right) \cup I_{2} \cup J_{2},
$$


onde a aplicação $\mathcal{F}_{2}^{\prime \prime}$ restrita em $\Delta$ é $\alpha$-extensiveis e todos os domínios da partição $\eta_{2}^{\prime \prime}$ têm sua extensão contida em $\hat{I}_{1}$ ou $\hat{J}_{1}$. Ainda mais, $\hat{I}_{2} \subset I_{1}, \hat{J}_{2} \subset J_{1}$.

\section{Dividindo $I_{1}^{-k}$ e $J_{1}^{-k}$}

Refinados os dois intervalos $I_{1}$ e $J_{1}$, refinamos também os intervalos do tipo $I_{1}^{-k}, J_{1}^{-k}$ usando a pré-imagem de intervalos de $\eta_{2}^{\prime \prime}$ por $\chi$. Então temos uma partição,

$$
I_{1}^{-k} \cup J_{1}^{-k}=(\cup \Delta) \cup\left(\cup I_{1}^{-k}\right) \cup\left(\cup J_{1}^{-k}\right) \cup I_{2}^{-k} \cup J_{2}^{-k}
$$

\section{Extendibilidade:}

Podemos ter algum $\Delta$, provavelmente perto do bordo de $I_{1}$, cuja estensão não esteja contida na imagem de $\hat{\chi}\left(\hat{I}_{1}^{-k}\right)$, visto que não temos a extendibilidade de $\chi$ neste caso. Para estes $\Delta$ aplicamos o Refinamento no Bordo e assim conseguimos todos os $\Delta \operatorname{em} I_{1}^{-k}$ extensíveis. Como $I_{2}$ tem sua extensão contida em $I_{1}$ e como $\chi: I_{1}^{-k} \rightarrow I_{1}$ é monótona, temos que o intervalo $I_{2}^{-k}$ construido em $I_{1}^{-k}$ possue uma extensão contida em $I_{1}^{-k}$ e que é levado pela aplicação $\chi$ sobre $\hat{I}_{2}$. Repetimos este processo infinitas vezes em todos os $I_{1}^{-k}$ e $J_{1}^{-k}$ construídos e assim obtemos uma partição em $I_{1}^{-k} \cup J_{1}^{-k}$ :

$$
I_{1}^{-k} \cup J_{1}^{-k}=(\cup \Delta) \cup\left(\cup I_{2}^{-k}\right) \cup\left(\cup I_{2}^{-k}\right) \cup J_{2}^{-k} \cup C,
$$

onde $C$ é um conjunto de Cantor.

Dessa forma obtemos uma nova partição $\xi_{2}$ em $I_{0} \cup J_{0}$ :

$$
\xi_{2}: \quad I_{0} \cup J_{0}=(\cup \Delta) \cup\left(\cup I_{2}^{-k}\right) \cup\left(\cup J_{2}^{-k}\right) \cup I_{2} \cup J_{2} \cup C \text {. }
$$

\section{Hipótese da indução}


Seja $n \geq 2$, assumimos que existe uma partição $\xi_{n}$

$$
\xi_{n}: \quad I_{0} \cup J_{0}=(\cup \Delta) \cup\left(\cup I_{n}^{-k}\right) \cup\left(\cup J_{n}^{-k}\right) \cup I_{n} \cup J_{n} \cup C
$$

Assumimos também que existe uma aplicação $\mathcal{F}_{n}$ definida nessa aplicação satisfazendo as seguintes propriedades:

1. $\mathcal{F}_{n}$ aplica cada $\Delta$ difeomorficamente sobre $I_{0}$ ou $J_{0}$ e é $\alpha$-extensivel,

2. $\mathcal{F}_{n}$ aplica $I_{n} \cup J_{n}$ de modo unimodal em $I_{0}$ ou $J_{0}$ e é $\alpha$-extensível,

3. $\mathcal{F}_{n}$ aplica os intervalos $I_{n}^{-k}$ (respectivamente $J_{n}^{-k}$ ) difeomorficamente sobre $I_{n}$ (respectivamente $J_{n}$ ) e a imagem da extensão dessa aplicação é $\hat{I}_{n}$ (respectivamente. $\left.\hat{J}_{n}\right)$

4. A extensão de cada componente conexa de $\xi_{\text {n }}$ está contida em $\hat{I}$ ou $\hat{J}$.

\section{Passo $n+1$}

Estudaremos a contrução da partição apenas em $I_{n}$, pois em $J_{n}$ a contrução é análoga. Consideramos o ramo crítico $\phi_{n}$ definido em $I_{n}$. Então temos dois casos:

Caso 1: $\phi_{n}(c) \in \Delta_{*}$ : Vamos assumir que $\phi_{n}\left(I_{n}\right) \subset I_{0}$. Então podemos refinar $I_{n}$ usando a pré-imagem de intervalos de $\xi_{n}$ por $\phi_{n}$ e obtemos uma partição em $I_{n}$ :

$$
\eta_{n+1}: I_{n}=(\cup \Delta) \cup\left(\cup I_{n}^{-k}\right) \cup\left(\cup J_{n}^{-k}\right) \cup I_{n+1},
$$

onde $I_{n+1}=\phi_{n}^{-1}\left(\Delta_{*}\right)$. Como a aplicação $\phi_{n}\left(I_{n}\right)$ contém uma componente conexa de $\hat{\Delta}_{*} \backslash \Delta_{*}$, temos que $\phi_{n+1}=\mathcal{F}_{n \mid \Delta_{*}} \circ \phi_{n \mid I_{n+1}}$ tem sua imagem em $I_{0}$ ou $J_{0}$ e á $\alpha$-extensível. Usando, se for necessário, o Refinamento no bordo nos $\Delta$ perto de $\phi_{n}(c)$ pela pré-imagem 
de intervalos de $\xi_{n}$, podemos conseguir que todos os ramos definidos nos intervalos $\Delta$ em $\eta_{n+1}$ sejam $\alpha$-extensíveis. A seguir, refinamos os intervalos $I_{n}^{-k}, J_{n}^{-k}$ de $\xi_{n}$, usando a pré-imagem de intervalos de $\eta_{n+1}$ pela aplicação $\chi: I_{n}^{-k} \rightarrow I_{n}$ ou $\chi: J_{n}^{-k} \rightarrow J_{n}$. Usando, se for necessário, o Refinamento no bordo nos $\Delta$ perto do bordo de $I_{n}$, podemos conseguir que todos os ramos monôtonos definidos nos intervalos $\Delta$ em $I_{n}^{-k}$ sejam $\alpha$-extensíveis e obtemos uma partição em $I_{n}^{-k}$ :

$$
I_{n}^{-k}=(\cup \Delta) \cup\left(\cup I_{n}^{-k}\right) \cup\left(\cup J_{n}^{-k}\right) \cup\left(I_{n+1}^{-k}\right)
$$

Note que $\hat{I}_{n+1}^{-k}$ são levados difeomorficamente sobre $\hat{I}_{n+1}$, pois $\hat{I}_{n+1} \subset I_{n}$. Repetindo este processo em todos os intervalos do tipo $I_{n}^{-k}$ e $J_{n}^{-k}$, obtemos uma nova partição:

$$
\xi_{n+1}: \quad I_{0} \cup J_{0}=(\cup \Delta) \cup\left(\cup I_{n+1}^{-k}\right) \cup\left(\cup J_{n+1}^{-k}\right) \cup I_{n+1} \cup J_{n+1} \cup\left(C \cap\left(I_{0} \cup J_{0}\right)\right)
$$

Temos também que a aplicação definida nesta partição $\mathcal{F}_{n+1}$ é $\alpha$-extensível em $\Delta$, e aplica $\hat{I}_{n+1}^{-k}$ sobre $\hat{I}_{n+1}$, e $J_{n+1}^{-k}$ sobre $\hat{J}_{n+1}$. Ainda mais o ramo crítico $\phi_{n+1}$ definido em $I_{n+1}$ é $\alpha$-extensível tendo sua imagem em $I_{0} \cup J_{0}$.

Caso $2-\phi_{n}(c) \in I_{n}^{-k} \cup J_{n}^{-k}$ : A única diferença neste caso do caso anterior acontece apenas no ramo crítico $\phi_{n+1}$ definido em $I_{n+1}$. Assumiremos que $\phi_{n}(c) \in J_{n *}^{-k}$. Então $I_{n+1}=\phi_{n}^{-1}\left(J_{n *}^{-k}\right)$ e $\phi_{n+1}=\chi_{* \mid J_{n *}^{-k}} \circ \phi_{n \mid I_{n+1}}$, onde $\chi_{*}: J_{n *}^{-k} \rightarrow J_{n}$. Como a aplicação $\chi_{*}$ definida em $J_{n *}^{-k}$ possue uma extensão cuja imagem é $\hat{J}_{n}$, o ramo crítico $\phi_{n+1}$ aplica $I_{n+1}$ em $J_{n}$ Também existe $\hat{I}_{n+1} \supset I_{n+1}$ tal que $\phi_{n+1}: \hat{I}_{n+1} \rightarrow \hat{J}_{n}$ é unimodal. Então, ao refinar o intervalo $I_{n+1}$, usando a aplicação $\phi_{n+1 \mid I_{n+1}}$, precisamos restringir à pré-imagem de intervalos de $\xi_{n+1} \cap J_{n}$. Essa situação vai continuar até que, para algum $s, \phi_{n+s}(c)$ esteja contido em um $\Delta$. Entretanto, podemos continuar a partição de $I_{n+1}, J_{n+1}$ por 
indução e obtemos a partição final:

$$
\xi: \quad I_{0} \cup J_{0}=(\cup \Delta) \cup C,
$$

onde a aplicação $\mathcal{F}$ aplica cada intervalo $\Delta$ sobre $I$ ou $J$ e é $\alpha$-extensível. Isto concluí a prova da Proposição 6.1.1. 


\subsection{Hiperbolicidade no Caso Básico}

Na seção anterior mostramos que dada uma aplicação bimodal $f$ existe uma aplicação induzida $\mathcal{F}$ com uma partição $\xi$. Esta partição possui domínios de tipo $\Delta$ e um conjunto de cantor. Em geral esta aplicação induzida e partição podem ser construidas sem considerar a propriedade da distorçăo limitada em cada etapa da indução. Neste caso nào será necessário o processo de refinamento no bordo. Quando este conjunto de cantor tem medida de Lebesgue nula dizemos que a aplicação induzida $\mathcal{F}$ é aplicação de Markov. Dizemos também que uma aplicação de Markov é hiperbólica se $\mathcal{F}$ restrita a cada $\Delta$ tem distorção uniformemente limitada e existe uma constante $a>1$ tal que $|D \mathcal{F}(x)| \geq a$, para todo $x \in \cup_{\Delta \in \xi} \Delta$.

Lembre-se que na construçâo da aplicação induzida consideramos dois casos diferentes, dependendo de onde os valores críticos pertencem. Então diremos que a aplicação $\mathcal{F}$ é do caso básico se durante a contrução da aplicação induzida os valores críticos estiverem contidos em domínios do tipo $\Delta$ e é do caso não-básico se os valores críticos estiverem contidos em domínios $I_{n}^{-k}$ ou $J_{n}^{-k}$. Note que o caso não-básico inclui aplicações de tipo Fibonacci.

Primeiramente, mostraremos que as aplicações do caso básico apresentam hiperbolicidade. O Lema a seguir mostra que na verdade basta os valores críticos estiverem em $\Delta$ apenas infinitas vezes e não em toda etapa da construção da partição.

Sejam $I_{0}^{+}, I_{0}^{-}$componentes conexas de $\hat{I} \backslash I_{0}$ e $J_{0}^{+}, J_{0}^{-}$componentes conexas de $\hat{J} \backslash J_{0}$. Definimos:

$$
\alpha=\min \left\{\frac{\left|I_{0}^{+}\right|}{\left|I_{0}\right|}, \frac{\left|I_{0}^{-}\right|}{\left|I_{0}\right|}, \frac{\left|J_{0}^{+}\right|}{\left|J_{0}\right|}, \frac{\left|J_{0}^{-}\right|}{\left|J_{0}\right|}\right\} .
$$

Lembre-se que $\Delta_{0}, \Delta_{0}^{\prime}$ são intervalos externos definidos em $I_{0}$ e $\Delta_{1}, \Delta_{1}^{\prime}$ sào intervalos 
externos definidos em $J_{0}$. Definimos então:

$$
\gamma=\min \left\{\frac{\left|\Delta_{0}\right|}{\left|I_{0}\right|}, \frac{\left|\Delta_{0}^{\prime}\right|}{\left|I_{0}\right|}, \frac{\left|\Delta_{1}\right|}{\left|J_{0}\right|}, \frac{\left|\Delta_{1}^{\prime}\right|}{\left|J_{0}\right|}\right\}
$$

Neste contexto, diremos que $\alpha, \gamma$ e a propriedade da ordem de pontos críticos são gєometria inicial de $f$.

Suponha que $\phi_{n}: I_{n} \rightarrow I_{0}$. Assumimos que $\phi_{n}\left(I_{n}\right) \ni \Delta_{0}$. Definimos então dois intervalos $\Delta_{n_{1}}, \Delta_{n_{2}} \subset I_{n}$ como sendo pré-imagens de $\Delta_{0}$ por $\phi_{n}$.

Lema 6.2.1 Cada ramo crítico $\left.\phi_{n}\right|_{I_{n}},\left.\phi_{n}\right|_{J_{n}}$ é $\alpha$-extensível e existe $\alpha_{1}=\alpha_{1}(\alpha, \gamma)$ tal que o domínio da extensão $\hat{I}_{n}$ (resp. $\hat{J}_{n}$ ) é uma $\alpha_{1}$-vizinhança de $I_{n}$ (resp. $J_{n}$ ).

Prova: A extendibilidade dos ramos críticos vem direto da construção da partição mostrada na Proposição 6.1.1. Podemos escrever $\phi_{n}$ como sendo uma composição de forma $\phi_{n}=f_{n} \circ f$, onde $f_{n}$ é um difeomorfismo com distorção limitada controlada por $(1+1 / \alpha)^{2}$. Sendo assim, a prova deste Lema vem direto do Princípio macroscópico e da propriedade da ordem dos pontos críticos.

Lema 6.2.2 Existe $\sigma>0$ que depende apenas da geometria inicial, tal que

$$
\min \left\{\frac{\left|\Delta_{n_{1}}\right|}{\left|I_{n}\right|}, \frac{\left|\Delta_{n_{2}}\right|}{\left|I_{n}\right|}\right\}>\sigma
$$

Prova: Sabemos que $\phi_{n}=f_{n} \circ f$, onde $f_{n}$ é um difeomorfismo extensível. Seja $\Delta_{*}=f_{n}^{-1}\left(I_{0}\right)$, então pelo Princípio de Koebe existe uma constante $c_{1}$ tal que

$$
\frac{\left|f_{n}^{-1}\left(\Delta_{0}\right)\right|}{\left|\Delta_{*}\right|} \cdot \frac{\left|I_{0}\right|}{\left|\Delta_{0}\right|}>c_{1}(\alpha)
$$

e isso resulta

$$
\frac{\left|f_{n}^{-1}\left(\Delta_{0}\right)\right|}{\left|\Delta_{*}\right|}>c_{2}(\alpha, \gamma)
$$


Usando a propriedade da ordem do ponto crítico $c$ obtemos o resultado desejado

$$
\frac{\left|\Delta_{n_{i}}\right|}{\left|I_{n}\right|}=\frac{\left|f^{-1}\left(f_{n}^{-1}\left(\Delta_{0}\right)\right)\right|}{\left|f^{-1}\left(\Delta_{*}\right)\right|}>\sigma .
$$

Para algum $I_{n}^{-k}$ denotemos por $\mathcal{U}_{n}^{-k}$ a união de todo domínio do tipo $I_{n+1}^{-k}, J_{n+1}^{-k}$, construídos em $I_{n}^{-k}$.

Lema 6.2.3 Existe uma constante $\theta_{1} \in(0,1)$ que depende apenas da geometria inicial, tal que

$$
\frac{\left|\mathcal{U}_{n}^{-k}\right|}{\left|I_{n}^{-k}\right|}<\theta_{1}
$$

Prova: Do Lema anterior sabemos que a uniào de $I_{n+1}$ e todo intervalo do tipo $I_{n+1}^{-k}$ e $J_{n+1}^{-k}$ contidos em $I_{n}$ tem a medida relativa monor que $1-2 \sigma$. Do Lema 6.2.1, os difeomorfismos $\chi: I_{n}^{-k} \rightarrow I_{n i}$ são uniformemente extensíveis. Usando o lema anteior e o Principio de Koebe, obtemos que a medida relativa de domínios extensiveis $\Delta \mathrm{em}$ todo $I_{n}^{-k}$ é maior que uma constante $\beta>0$ determinada pela geometria inicial. Como $\mathcal{U}_{n}^{-k} \subset I_{n}^{-k}$ é contido em complementar da união de $\Delta$, temos que a medida relativa de $\mathcal{U}_{n}^{-k}$ em $I_{n}^{-k}$ é menor que $\theta_{1}=1-\beta$.

Este Lema junto com a Proposição 6.1.1 prova o Teorema 2.3.6 para o caso básico, pois a medida de $\mathcal{U}_{n}^{-k}$ reduz por um fator $\theta_{1}$ menor que 1. 


\subsection{Hiperbolicidade no Caso Não-Básico}

Lembre-se que as aplicações do caso básico têm uma aplicação induzida, na construção da qual os valores críticos pertencem a domínios dos ramos monótonos do tipo $\Delta$. Nesse caso, mostramos a hiperbolicidade da aplicação sem usar a propriedade do decaimento de geometria. Na verdade, na prova da Hiperbolicidade do caso básico, nem precisamos que os valores críticos pertencessem a domínios do tipo $\Delta$ em toda etapa da construção. Foi bastante se isso acontecesse apenas em um número infinito de vezes.

Vamos considerar nesta seção as aplicações não-básicas. Em função da hiperbolicidade do caso básico, podemos assumir que existe $n_{0} \geq 1$ tal que para todo $n \geq n_{0}$ os valores críticos $\phi_{n}(c), \phi_{n}(d)$ pertencem sempre a domínios do tipo $I_{n}^{-k}$ ou $J_{n}^{-k}$. A classe dessas aplicações é maior do que a de Fibonacci, ou seja, a nossa prova não se limitará apenas à classe de aplicações Fibonacci. Entretanto, precisaremos restringir o nosso trabalho às aplicações bimodais que exibem decaimento de geometria.

Lembre-se que para cada $n \geq 1$ temos uma partição:

$$
\xi_{n}: \quad I_{0} \cup J_{0}=(\cup \Delta) \cup\left(\cup I_{n}^{-k}\right) \cup\left(\cup J_{n}^{-k}\right) \cup I_{n} \cup J_{n}
$$

temos também uma aplicação $\mathcal{F}_{n}$ definida nesta partição. Como os valores críticos de $\mathcal{F}_{n-1}$ pertencem a domínios do tipo $I_{n-1}^{-k}, J_{n-1}^{-k}, \mathcal{F}_{n}$ restrita a domínios $I_{n}$ e $J_{n}$, isto é, os ramos críticos $\phi_{n}$ aplicam seus domínios em $I_{n-1}$ ou $J_{n-1}$ de modo unimodal. E $\mathcal{F}_{n}$ restrita a domínios do tipo $\Delta$, denotado por $\mathcal{F}_{\Delta}$, aplicam seus domínios difeomorficamente sobre $I_{0}$ ou $J_{0}$. E os domínios $I_{n}^{-k}$ e $J_{n}^{-k}$ são aplicados difeomorficamente sobre $I_{n}$ e $J_{n}$ respectivamente pela aplicação $\chi$. Lembre-se que os intervalos $I_{n}^{-k}, J_{n}^{-k}$ são domínios de primeira entrada para os intervalos $I_{n}, J_{n}$ respectivamente e cada destes intervalos tem 
sua extensão do tipo $I_{n-1}^{-k}, J_{n-1}^{-k}$ tais que a aplicação $\chi$ tem sua extensão definida nestes intervalos, cuja imagem é $I_{n-1}, J_{n-1}$ respectivamente.

Nesta seção assumimos que $f$ exibe decaimento exponencial de geometria, ou seja, existem constantes $A>0$ e $\lambda>1$ tais que para cada $n \geq 1$, satisfazem-se o seguinte:

$$
\frac{\left|I_{n}\right|}{\left|I_{n-1}\right|} \leq \frac{1}{1+2 A \lambda^{n}} \quad \text { e } \quad \frac{\left|J_{n}\right|}{\left|J_{n-1}\right|} \leq \frac{1}{1+2 A \lambda^{n}}
$$

Lema 6.3.1 Para todo $I_{n}^{-k}, J_{n}^{-k} \in \xi_{n}$, os difeomorfismos $\chi: I_{n}^{-k} \rightarrow I_{n}$ e $\chi: J_{n}^{-k} \rightarrow J_{n}$ são $A \lambda^{n}$-extensíveis e têm distorçâo limitada por $\left(1+\frac{1}{A \lambda^{n}}\right)^{2}$.

Prova: Da Proposição 6.1.1 podemos ver que todos os intervalos do tipo $I_{n}^{-k}, J_{n}^{-k}$ são restrições dos intervalos $I_{n-1}^{-k}, J_{n-1}^{-k}$ respectivamente e que esses intervalos são aplicados por $\chi$ sobre $I_{n-1}, J_{n-1}$ enquanto os intervalos $I_{n}^{--k}, J_{n}^{-k}$ são aplicados por mesma aplicação sobre $I_{n}, J_{n}$. Como $I_{n-1}, J_{n-1}$ são, pela hipótese do decaimento de geometria, $A \lambda^{n-}$ vizinhanças de $I_{n}, J_{n}$ respectivamente, usando o Lema 3.1.1, conclui-se a prova.

Observamos que $\phi_{n}(c)$ e $\phi_{n}(d)$ são contidos em algum dos intervalos do tipo $I_{n}^{-k}$ ou $J_{n}^{-k}$, denotamo-los por $\tilde{C}_{n}$ e $\tilde{D}_{n}$ respectivamente. Estes intervalos terão suas extensões do tipo $I_{n-1}^{-k}$ ou $J_{n-1}^{-k}$, entào vamos denotá-los por $C_{n}$ e $D_{n}$ respectivamente. Lembre-se que os intervalos $I_{n+1}$ e $J_{n+1}$ são definidos como sendo pré-imagens de $\tilde{C}_{n}$ e $\tilde{D}_{n}$ pelos ramos críticos $\phi_{n}$. Vamos definir as vizinhas desses intervalos do seguinte modo:

$$
W_{n+1}(c)=\phi_{n}^{-1}\left(C_{n}\right) \text { e } W_{n+1}(d)=\phi_{n}^{-1}\left(D_{n}\right)
$$

Lema 6.3.2 Para todo $n \geq 1$, as aplicaçôes $\phi_{n}$ possuem $A \lambda^{n-1}$-extensôes unimodais $\hat{\phi}_{n}$. Estas extensôes estão definidas, respectivamente, nos intervalos $W_{n}(c)$ e $W_{n}(d)$ e 
são tais que $\hat{\phi}_{n}\left(\partial W_{n}(c)\right)$ e $\hat{\phi}_{n}\left(\partial W_{n}(d)\right)$ estão em $\partial I_{n-2}$ ou $\partial J_{n-2}$ para $n \geq 2$. Além disso, para cada $n \geq 1$ existem aplicações monótonas $\Phi_{n}$ tais que

$$
\hat{\phi}_{n}=\Phi_{n} \circ f
$$

com

$$
\left(1+\frac{1}{A \lambda^{n-1}}\right)^{-2} \leq \frac{D \Phi_{n}(f(x))}{D \Phi_{n}(f(y))} \leq\left(1+\frac{1}{A \lambda^{n-1}}\right)^{2}
$$

para $x, y \in I_{n}$ ou $x, y \in J_{n}$.

Prova: Vamos provar o Lema pela indução em $n$ apenas para os ramos críticos definidos ao redor de $c$, visto que a prova do outro caso será análoga. Lembre-se que temos a partição inicial definida por $\xi_{0}:=[0,1]=I_{0} \cup J_{0} \cup(\cup \Delta)$ e a aplicação $\mathcal{F}_{0}$ definida nesta partição. $\mathcal{F}_{0}$ aplica cada um dos $\Delta$ sobre $l_{0}$ ou $J_{0}$ monotonamente e com distorção uniformemente limitada. Para $n=1$ temos que $\phi_{\left.1\right|_{J_{1}}}=\mathcal{F}_{0 \mid \Delta_{c}} \circ f$, onde $f(c) \in \Delta_{c}$ e, assim, o Lema segue. Assumimos que o Lema é verdadeiro para $n$ e vamos mostrá-lo para $n+1$. Temos que $\phi_{n+1 \mid I_{n+1}}=\mathcal{F}_{n \mid \bar{C}_{n}} \circ \phi_{n \mid I_{n+1}}$. Pela hipótese da indução, existe um difeomorfismo $\Phi_{n}$ tais que $\phi_{n}=\Phi_{n} \circ f$. Como $\mathcal{F}_{n \mid \tilde{C}_{n}}$ é monótona, definindo $\Phi_{n+1}=\mathcal{F}_{n \mid \tilde{C}_{n}} \circ \Phi_{n}$, temos que

$$
\phi_{n+1}=\Phi_{n+1} \circ f
$$

Como $W_{n}(c)=\phi_{n-1}^{-1}\left(C_{n-1}\right)$ e $\mathcal{F}_{n-1}$ tem sua extensão $\hat{\mathcal{F}}_{n-1}$ definida em $C_{n-1}$, resulta que $\phi_{n}$ têm sua extensão $\hat{\phi}_{n}$ definida em $W_{n}(c)$ e $\hat{\phi}_{n}\left(\partial W_{n}(c)\right)$ está em $\partial I_{n-2}$ ou $\partial J_{n-1}$, enquanto que a imagem de $\phi_{n}$ está contida em $I_{n-1} \cup J_{n-1}$. Como $\frac{\left|I_{n-1}\right|}{\left|I_{n-2}\right|}$ e $\frac{\left|J_{n-1}\right|}{\left|J_{n-2}\right|}$ são majorados por $\left(1+2 A \lambda^{n-1}\right)^{-1}$ o Lema segue.

Corolário 6.3.1 Existe um número real $A_{1}>0$ tal que 
1. para $n \geq 0$ e $x \in I_{n}$ verifica-se que

$$
\left(1+\frac{A_{1}}{\lambda^{n-1}}\right)^{-3}\left|D^{2} \phi_{n}(c)\right||x-c| \leq\left|D \phi_{n}(x)\right| \leq\left(1+\frac{A_{1}}{\lambda^{n-1}}\right)^{3}\left|D^{2} \phi_{n}(c)\right||x-c| .
$$

2. para $n \geq 0$ e $x \in J_{n}$ verifica-se que

$$
\left(1+\frac{A_{1}}{\lambda^{n-1}}\right)^{-3}\left|D^{2} \phi_{n}(d)\right||x-d| \leq\left|D \phi_{n}(x)\right| \leq\left(1+\frac{A_{1}}{\lambda^{n-1}}\right)^{3}\left|D^{2} \phi_{n}(d)\right||x-d| .
$$

Prova: Vamos provar apenas a primeira afirmação, visto que a prova da segunda é análoga. Pelo Lema 6.3.2 temos que $\phi_{n}$ é $A \lambda^{n-1}$-extensivel e $\phi_{n}=\Phi_{n} \circ f$, onde $\Phi_{n}$ é um difeomorfismo $A \lambda^{n-1}$-extendivel e, portanto, com distorçào limitada por $\left(1+\frac{1}{A \lambda^{n-1}}\right)^{2}$. Como

$$
\frac{\left|I_{n}\right|}{\left|I_{n-1}\right|} \leq \frac{1}{1+2 A \lambda^{n}}
$$

em particular $\left|I_{n}\right| \leq\left(1+2 A \lambda^{n}\right)^{-1}\left|I_{0}\right|$. Sendo 2 a ordem do ponto crítico $c$ concluímos que existe constante $A_{0}<\infty$ tal que para todo $x \in I_{n}$ verifica-se que

$$
\left(1+\frac{A_{0}}{\lambda^{n-1}}\right)^{-1}\left|D^{2} f(c)\right||x-c| \leq|D f(x)| \leq\left(1+\frac{A_{0}}{\lambda^{n-1}}\right)\left|D^{2} f(c) \|\right| x-c \mid
$$

Como $\left|D \phi_{n}(x)\right|=\left|D \Phi_{n}(f(x))\right||D f(x)| \mathrm{e}$

$$
\left(1+\frac{1}{A \lambda^{n-1}}\right)^{-2} \leq \frac{\left|D \Phi_{n}(f(x))\right|}{\left|D \Phi_{n}(f(c))\right|} \leq\left(1+\frac{1}{A \lambda^{n-1}}\right)^{2},
$$

temos uma constante $A_{1}<\infty$ tal que

$$
\left(1+\frac{A_{1}}{\lambda^{n-1}}\right)^{-3}\left|D \Phi_{n}(f(c))\right|\left|D^{2} f(c)\right||x-c| \leq\left|D \phi_{n}(x)\right| \leq\left(1+\frac{A_{1}}{\lambda^{n-1}}\right)^{3}\left|D \Phi_{n}(f(c)) \| D^{2} f(c)\right||x-c| .
$$

Como $D^{2} \phi_{n}(c)=D \Phi_{n}(c) \cdot D^{2} f(c)$, o Lema segue. 
Corolário 6.3.2 Existe uma constante $A_{2}<\infty$ tal que para todo $n \geq 0$ verifica-se que

$$
\frac{\left|I_{n+1}\right|}{\left|W_{n+1}(c)\right|} \leq \frac{A_{2}}{\left(1+2 A \lambda^{n}\right)^{1 / 2}}, \quad e \quad \frac{\left|W_{n+1}(c)\right|}{\left|I_{n}\right|} \leq \frac{A_{2}}{\lambda^{n / 4}}
$$

O mesmo verifica-se também para os intervalos definidos em volta do ponto crítico d.

Prova: Vamos mostrar apenas as primeiras duas afirmações, pois as demais provamse de maneira análoga. Lembre-se que $W_{n+1}(c)=\phi_{n}^{-1}\left(C_{n}\right)$ e $I_{n+1}=\phi_{n}^{-1}\left(\tilde{C}_{n}\right)$. Usando o Corolário 6.3.1 temos que

$$
\frac{\left|I_{n+1}\right|}{\left|W_{n+1}(c)\right|} \leq\left(1+\frac{A_{1}}{\lambda^{n}}\right)^{3}\left(\frac{\left|\tilde{C}_{n}\right|}{\left|C_{n}\right|}\right)^{1 / 2}
$$

Como $C_{n}$ e $\tilde{C}_{n}$ são pré-imagens de $I_{n-1}$ e $I_{n}$ (ou $J_{n-1}$ e $J_{n}$ ) respectivamente pelo difeomorfismo $\chi$, usando o Princípio Macroscópico de Köbe, obtemos

$$
\frac{\left|I_{n+1}\right|}{\left|W_{n+1}(c)\right|} \leq \frac{A_{2}}{\left(1+2 A \lambda^{n}\right)^{1 / 2}}
$$

para uma constante $A_{2}<\infty$. Isto prova a primeira afirmação.

Para demostrar a segunda afirmação precisamos considerar duas situações possíveis. Para ambos casos assumiremos que $C_{n} \subset I_{n-1}$ e $\phi_{n-1}: I_{n-1} \rightarrow j_{n-2}$ (em demais situações a prova será análoga):

1. $\phi_{n-1}\left(C_{n}\right) \neq J_{n-1}$. Lembre-se que o intervalo $C_{n}$ é aplicado pelo difeomorfismo $\chi$ sobre $I_{n-1}$ ou $J_{n-1}$ dependendo do caso. Afirmamos que $I_{n-1}$ é uma $A \lambda^{n-1}$-vizinhança de $C_{n}$. Pela hipótese temos que $\phi_{n-1}\left(C_{n}\right) \subset J_{n-2} \backslash J_{n-1}$ e que $\phi_{n-1}\left(C_{n}\right)$ é agora um domínio de primeira entrada para $I_{n-1}$ ou $J_{n-1}$. Então existe um intervalo $T$ como sendo uma extensão monótona de $\phi_{n-1}\left(C_{n}\right)$ cuja imagem é sobre $I_{n-2}$ oul $J_{n-2}$. Pelo decaimento de geometria e Princípio de Köbe, temos que $T$ é uma $A \lambda^{n-1}$-vizinhança de $\phi_{n-1}\left(C_{n}\right)$. Usando o Corolário 6.3.1, $C_{n}$ possui uma $A \lambda^{\frac{n-1}{2}}$-vizinhança contida em uma 
das componentes conexas de $I_{n-1} \backslash\{c\}$ pelo menos no lado oposto do ponto crítico. Isto prova a afirmação.

Então, usando o Corolário 6.3.1, verifica-se que

$$
\begin{aligned}
\frac{\left|W_{n+1}(c)\right|}{\left|I_{n}\right|} & \leq\left(1+\frac{A_{1}}{\lambda^{n-1}}\right)^{3}\left(\frac{C_{n}}{I_{n-1}}\right)^{1 / 2} \\
& \leq\left(1+\frac{A_{1}}{\lambda^{n-1}}\right)^{3}\left(\frac{1}{1+2 A \lambda^{\frac{n-1}{2}}}\right)^{1 / 2}
\end{aligned}
$$

Disto segue que existe uma constante $A_{2}<\infty$ que satisfaz o Lema.

2. $\phi_{n-1}\left(C_{n}\right)=J_{n-1}$. Nesta situação temos que $\phi_{n-1}\left(I_{n-1}\right)$ possui uma das componentes conexas de $J_{n-2} \backslash J_{n-1}$. Em outras palavras, $\phi_{n-1}\left(I_{n-1}\right)$ possui uma $A \lambda^{n-1}$-vizinhança de $\phi_{n-1}\left(C_{n}\right)$ num lado deste que não contém $\phi_{n-1}(c)$. Então, usando o Corolário 6.3.1 temos que $I_{n-1}$ é uma $\left(A \lambda^{n-1}\right)^{1 / 2}$-vizinhança de $C_{n}$. Usando novamente o Corolário 6.3 .1 pela aplicação $\phi_{n \mid I_{n}}$ temos que

$$
\frac{\left|W_{n+1}(c)\right|}{\left|I_{n}\right|} \leq \frac{A_{2}}{\lambda^{n / 4}}
$$

Avaliaremos em seguinda, qual a proporção que os intervalos $\Delta$ que surgem na construção de cada etapa da partição $\xi$ representa em $[0,1]$. Para isso, definimos:

$$
\Gamma_{n}:=\cup_{\Delta \in \xi_{n}} \Delta, \quad \Gamma_{n}(c):=\Gamma_{n} \cap W_{n}(c) \text { e } \Gamma_{n}(d):=\Gamma_{n} \cap W_{n}(d) .
$$

Definimos também

$$
\delta_{n}:=\min \left\{\frac{\left|\Gamma_{n}(c)\right|}{\left|W_{n}(c)\right|}, \frac{\left|\Gamma_{n}(d)\right|}{\left|W_{n}(d)\right|}\right\}
$$

Lema 6.3.3 Existe um número real $A_{3}>0$ tal que para todo $n \geq 0$ verifica-se que

$$
\delta_{n+2} \geq \frac{\lambda^{n / 4}}{A_{3}+\lambda^{n / 4}} \delta_{n+1} .
$$


Prova: Observemos que para cada $I_{n+1}^{-k}$ da partição $\xi_{n+1}$ existem intervalos $I_{n}^{-k}, W_{n+1}^{-k}(c)$ tais que

$$
I_{n+1}^{-k} \subset W_{n+1}^{-k}(c) \subset I_{n}^{-k}
$$

Cada uma destas triplas é aplicada por um difeomorfismo $\hat{\chi}$ sobre $I_{n+1} \subset W_{n+1}(c) \subset$ $I_{n}$. Pelo corolário 6.3.2 estes difeomorfismos têm em $W_{n+1}^{-k}(c)$ distorção limitada por $\left(1+\frac{B}{\lambda^{n / 4}}\right)^{2}$, onde $B<\infty$ independe de $n$. Cada intervalos $W_{n+1}^{-k}(c)$ contém a respectiva pré-imagem de $\Gamma_{n+1}(c)$ que denotamos por $\Gamma_{n+1}^{\cdots k}(c)$. Temos então que

$$
\frac{\left|\Gamma_{n+1}^{-k}(c)\right|}{\left|W_{n+1}^{-k}(c)\right|} \geq\left(1+\frac{B}{\lambda^{n / 4}}\right)^{-2} \frac{\left|\Gamma_{n+1}(c)\right|}{\left|W_{n+1}(c)\right|}
$$

Isto e o fato das pré-imagens de $\Gamma_{n+1}(c)$ serem disjuntas, temos que

$$
\frac{\left|\Gamma_{n+1} \cap I_{n}^{-k}\right|}{\left|I_{n}^{-k}\right|} \geq\left(1+\frac{B}{\lambda^{n / 4}}\right)^{-2} \delta_{n+1}
$$

Em particular, consideramso duas triplas de intervalos $\tilde{C}_{n+1} \subset W_{n+1}^{-k}(c) \subset C_{n+1}$ e temos

$$
\frac{\left|\Gamma_{n+1} \cap C_{n+1}\right|}{\left|C_{n+1}\right|} \geq\left(1+\frac{B}{\lambda^{n / 4}}\right)^{-2} \delta_{n+1}
$$

Lembre-se que os intervalos $I_{n+2}$ e $W_{n+2}(c)$ são definidos como sendo pré-imagens de $\dot{C}_{n+1}$ e $C_{n+1}$ respectivamente por $\phi_{n+1}$. Tambem ficam definidos $\Gamma_{n+2}(c)=\phi_{n+1}^{-1}\left(\Gamma_{n+1} \cap C_{n+1}\right)$. Então pelo Corolário 6.3.1 existe uma constante $A>0$ tal que

$$
\frac{\left|\Gamma_{n+2}(c)\right|}{\left|W_{n+2}(c) \backslash I_{n+2}\right|} \geq A \frac{\left|\Gamma_{n+1} \cap C_{n+1}\right|^{1 / 2}}{\left|C_{n+1}\right|^{1 / 2}}
$$

Pelo Lema 6.3.2 temos que

$$
\frac{\left|I_{n+2}\right|}{\left|W_{n+2}(c)\right|} \leq \frac{A_{2}}{\left(1+2 A \lambda^{n+1}\right)^{1 / 2}}
$$


Logo concluimos

$$
\begin{aligned}
\frac{\left|\Gamma_{n+2}(c)\right|}{\left|W_{n+2}(c)\right|} & =\frac{\left|\Gamma_{n+2}(c)\right|}{\left|W_{n+2}(c) \backslash I_{n+2}\right|} \frac{\left|W_{n+2}(c) \backslash I_{n+2}\right|}{\left|W_{n+2}(c)\right|} \\
& \geq A\left(1+\frac{B}{\lambda^{n / 4}}\right)^{-1}\left(1-\frac{A_{2}}{\left(1+2 A \lambda^{n+1}\right)^{1 / 2}}\right) \delta_{n+1} .
\end{aligned}
$$

O mesmo verifica-se para $\left|\Gamma_{n+2}(d)\right| /\left|W_{n+2}(d)\right|$. Como o valor $A\left(1-A_{2} /\left(1+2 A \lambda^{n+1}\right)^{1 / 2}\right)$ é maior do que uma constante positiva, segue que existe uma constante $A_{3}<\infty$ satisfazendo o Lema.

Corolário 6.3.3 Existe uma constante $\theta>0$ tal que $\delta_{n}>\theta, \quad \forall n \geq 1$.

Prova: O Lema 6.3.3 garante a existência de uma constante $A_{3}<\infty$ tal que

$$
\delta_{n+2} \geq \frac{\lambda^{n / 4}}{A_{3}+\lambda^{n / 4}} \dot{\delta}_{n+1}
$$

Então para todo $n \geq 1$ temos que

$$
\delta_{n+1} \geq \Pi_{i=1}^{n} \frac{\lambda^{i / 4}}{A_{3}+\lambda^{i / 4}} \delta_{1}
$$

Mas

$$
\log \left(\Pi_{i=1}^{n} \frac{\lambda^{i / 4}}{A_{3}+\lambda^{i / 4}}\right)=-\sum_{i=1}^{n} \log \left(\frac{A_{3}}{\lambda^{i / 4}}+1\right) .
$$

Como $\log (1+x)<x$ para todo $x>0$, resulta que a soma acima é maior que um número negativo mas real. Logo existe uma constante $\theta>0$ tal que $\delta_{n}>\theta$.

Mostraremos a seguir que a aplicação induzida $\mathcal{F}$ está definida num subconjunto de medida total de $[0,1]$. Disto mostraremos também a hiperbolicidade desta aplicação. Definimos

$$
S_{n}:=\left(\cup I_{n}^{-k}\right) \cup\left(\cup J_{n}^{-k}\right) \cup I_{n} \cup J_{n}
$$


Lema 6.3.4 Seja $S=\cap_{n=0}^{\infty} S_{n}$, o dominio de $\mathcal{F}$. Então $|S|=0$.

Prova: Do corolário 6.3 .3 existe $\beta<1$ tal que

$$
\frac{\left|S \cap W_{n}(c)\right|}{\left|W_{n}(c)\right|} \leq \beta, \frac{\left|S \cap W_{n}(d)\right|}{\left|W_{n}(d)\right|} \leq \beta
$$

Dado $x \in S$, existem intervalos arbitrariamente pequenos do tipo $W_{n}^{-k}(c)$ ou $W_{n}^{-k}(d)$ que contêm $x$. Como estes intervalos são aplicados em $W_{n}(c)$ ou $W_{n}(d)$ por um difeomorfismo com distorção limitada, verifica-se que existe uma constante $\beta^{\prime}<1$ tal que

$$
\frac{\left|S \cap W_{n}^{-k}(c)\right|}{\left|W_{n}^{-k}(c)\right|} \leq \beta^{\prime} \quad \text { e } \frac{\left|S \cap W_{n}^{-k}(d)\right|}{\left|W_{n}^{-k}(d)\right|} \leq \beta^{\prime} .
$$

Logo pelo Teorema da Densidade de Lebesgue, $S$ tem medida nula.

Este Lema junto com a Proposição 6.1.1 prova o Teorema 2.3.6 para o caso não básico.

Observação: Sabe-se que a aplicação Fibonacci bimodal possue $\omega(c), \omega(d)$ idênticos, contendo pontos críticos $c, d$ e ainda minimais. Foi provada então a hiperbolicidade da aplicação Fibonacci bimodal usando a minimalidade desses conjuntos. Nessa prova não foi necessário mostrar a extendibilidade dos ramos monôtonos na construção da aplicação induzida, em outras palavras, não foi usado o Refinamento no Bordo que nós usamos no contexto. Como os pontos críticos estão contidos em $\omega(c) \cup \omega(d)$, cuja distância a $\partial I_{0} \cup \partial J_{0}$ é maior do que alguma constante positiva, resulta que existe $\alpha>0$ tal que os ramos de $\mathcal{F}$ ou mesmo os de $\mathcal{F}^{N}$ para $N \geq 1$ sejam $\alpha$-extensíveis e, por isso, com distorção uniformemente limitada.

Em [Mar] foi provado que, quando o conjunto $\omega(c)$ não é minimal, existem dois intervalos $U \subset V$ e uma aplicação de primeiro retorno a $U$ definida em um conjunto de medida total tal que restrita a cada componente conexa do seu domínio existe uma 
extensão cuja imagem contém $V$. Existem também uma seqüência de intervalos $K_{n}$, com $K_{n} \supset K_{n+1}$ e $K_{n} \cap V=\phi$, e uma seqüência de números inteiros positivos $t_{n}$ tais que, para cada $n \geq 1, t_{n}$ é o tempo de primeira entrada do ponto crítico $c$ no intervalo $K_{n}$. Essa situação verifica que o caso é básico segundo a classificação deste contexto. 


\section{Referências Bibliográficas}

[B] Beardon, A.: Iteration of rational functions- Graduate texts in mathematics. Springer-Verlag (1991)

[BKNV] Bruin, H., Keller, G., Nowicki, T., Van Strein, S.: Wild cantor attractors existAnn. Math. 143, 97-130 (1996)

[GS1] Graczyk, J., Świątek, G.: Induced expansion for quadratic polynomialsAnn.Scient.Éc. Norm.Sup. 2,399-482 (1996)

[GS2] Graczyk, J., Świątek, G.: Hyperbolicity in the real quadratic family- Ann. Math $146,1-52(1997)$

[GS3] Graczyk, J., Świątek, G.: The Real Fatou Conjecture-Annals of Mathematics Studies 144 (1998)

[HK] Hofbauer, F., Keller, G.: Some remarks on recent results about S-unimodal mapsAnn. Inst. H. Poincaré Phys.Théor 53, 413-425 (1990)

[L] Lyubich, M.: Combinatorics, geometry and attractors of quasiquadratic maps.- Ann. Math. 140, 347-404 (1994) 
[L1] Lyubich, M.: Ergodic Theory for Smooth One-Dimensional Dynamical System-IMS Stony Brook Preprint (1991)

[LM] Lyubich, M. Milnor, J.: The Fibonacci unimodal map-Journal of the American Mathematical Society 6, Number 2, 425-457 (1993)

[LV] Lehto, O., Virtanen, K.: Quasiconformal Mappings in the Plane- Springer-Verlag, Berlin-Heidelberg-New York (1973)

[M] Milnor, J.: On the concept of attractor-Comm. math. phys. 99, 177-195 (1985) and 102, $517-519(1985)$

[Mar] Martens, M.: Distortionresults and invariant Cantor sets of unimodal mapsErgodic Theory and Dynamical Systems 14, 331-349 (1994)

[MS] de Melo, W., van Strein, S.: One-dimensional dynamics-Ergebnisse der Mathematic und ihrer Grenzgebiete (Berlin: Springer) (1993)

[S] Singer, D.: Stable orbits and bifurcations of maps of the interval-SIAM J.-Appl. Math. 35, 260-267 (1978)

[SV] Świątek, G., Vargas, E.: Decay of geometry in the cubic family-Ergodic Theory and Dynamical Systems 18, 1311-1329 (1998)

[SV2] van Strein, S., Vargas, E.: Real bounds,ergodicity and negative Schwarzian for multimodal maps- a aparecer no Journal of the American Mathematics Society. 